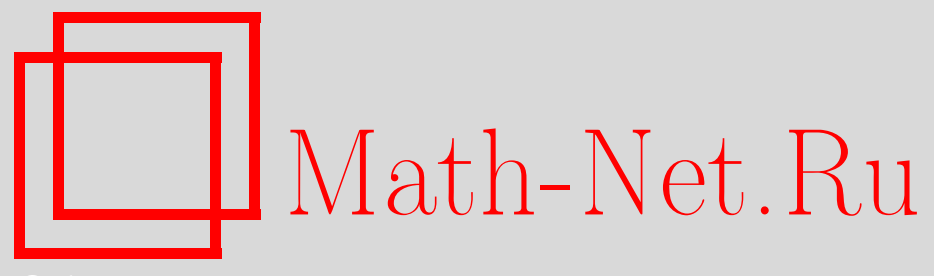

Г. М. Губреев, Ю. Д. Латушкин, Функциональные модели несамосопряженных операторов, сильно непрерывные полугруппы и матричные веса Макенхаупта, Изв. РАН. Сер. матем., 2011, том 75, выпуск 2, 69-126

DOI: https://doi.org/10.4213/im4098

Использование Общероссийского математического портала Math-Net.Ru подразумевает, что вы прочитали и согласны с пользовательским соглашением http://www . mathnet.ru/rus/agreement

Параметры загрузки:

IP : 52.90 .164 .192

26 апреля 2023 г., $16: 39: 52$

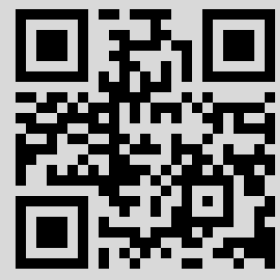


Г. М. Губреев, Ю. Д. Латушкин

\section{Функциональные модели несамосопряженных операторов, сильно непрерывные полугруппы и матричные веса Макенхаупта}

Рассмотрены неограниченные непрерывно обратимые операторы $A, A_{0}$ в гильбертовом пространстве $\mathfrak{H}$ такие, что оператор $A^{-1}-A_{0}^{-1}$ конечномерен. При условии, что $\sigma\left(A_{0}\right)=\varnothing$ и полугруппа $V_{+}(t):=\exp \left\{i A_{0} t\right\}, t \geqslant 0$, принадлежит классу $C_{0}$, формулируются критерии того, что полугруппы $U_{ \pm}(t):=\exp \{ \pm i A t\}, t \geqslant 0$, также принадлежат классу $C_{0}$. Указаны приложения к теории периодических в среднем функций. Исследования опираются на функциональные модели несамосопряженных операторов и на технику матричных весов Макенхаупта.

Библиография: 27 наименований.

Ключевые слова: $C_{0}$-полугруппы, функциональные модели несамосопряженных операторов, матричные веса Макенхаупта, гильбертовы пространства целых функций.

\section{Введение}

Настоящая статья посвящена решению следующей задачи теории возмущений однопараметрических полугрупп операторов в гильбертовых пространствах. Пусть $A, A_{0}$ - линейные неограниченные плотно заданные и непрерывно обратимые операторы в сепарабельном гильбертовом пространстве $\mathfrak{H}$, которые связаны равенством

$$
A^{-1} h=A_{0}^{-1} h+\sum_{k=1}^{n}\left(h, f_{k}\right) g_{k}, \quad h \in \mathfrak{H},
$$

где $f_{k}, g_{k} \in \mathfrak{H}, 1 \leqslant k \leqslant n$, и скобки обозначают скалярное произведение в $\mathfrak{H}$. Далее предполагается, что системы векторов $\left\{f_{k}\right\}_{k=1}^{n},\left\{g_{k}\right\}_{k=1}^{n}$ линейно независимы и оператор $A_{0}$ удовлетворяет следующим условиям:

1) он не имеет спектра и генерирует $C_{0}$-полугруппу $V_{+}(t):=\exp \left\{i A_{0} t\right\}, t \geqslant 0$;

$2)$ целая оператор-функция $\left(A_{0}-z I\right)^{-1}$ имеет конечный экспоненциальный тип роста.

Класс операторов $A_{0}$, которые удовлетворяют указанным условиям 1$), 2$ ), будем обозначать через $\Sigma^{(\exp )}$.

Работа первого автора выполнена при финансовой поддержке в рамках Госбюджетной темы Украины (грант № 0107U000937). Работа второго автора выполнена при поддержке Национального научного фонда США (гранты DMS-0405526, DMS-0338743, DMS-0354339, DMS-0754705). Оба автора получали поддержку CRDF (грант UP1-2567-0D-03). 
Конечномерные возмущения (0.1), вообще говоря, существенно изменяют свойства оператора $A_{0} \in \Sigma^{(\exp )}$. В частности, к операторам $A$ неприменимы общие теоремы теории возмущений однопараметрических полугрупп [1]-[7]. Поэтому значительный интерес представляет задача об описании таких $n$-мерных возмущений, что оператор $A$, связанный с $A_{0}$ равенством $(0.1)$, генерирует $C_{0}$-полугруппу $U_{+}(t):=\exp \{i A t\}$ или $C_{0}$-полугруппу $U_{-}(t):=\exp \{-i A t\}, t \geqslant 0$.

В настоящей статье мы выделяем класс конечномерных возмущений вида (0.1) и развиваем технику, основанную на использовании функциональных моделей несамосопряженных операторов и матричных весов Макенхаупта, которая позволит решить сформулированные задачи о полугруппах $U_{ \pm}(t)$. Упомянутый класс конечномерных возмущений оператора $A_{0} \in \Sigma^{(\exp )}$ определяется следующим образом. По оператору $A_{0}$ и векторам $\left\{g_{k}\right\}_{k=1}^{n}$ построим $n$-мерную строку

$$
\mathcal{A}_{0}(z, h):=\operatorname{row}\left\{\left(A_{0}\left(A_{0}-z I\right)^{-1} g_{k}, h\right)\right\}_{k=1}^{n}, \quad h \in \mathfrak{H},
$$

и примем следующее определение.

ОПРЕДЕЛЕНИЕ 0.1. Пусть операторы $A, A_{0}$ связаны равенством (0.1). Будем говорить, что $A-w$-возмущение ранга $n$ оператора $A_{0} \in \Sigma^{(\exp )}$, если существует $(n \times n)$-матричный $\left(A_{2}\right)$-вес $w^{2}($ см. $\S 1)$ такой, что выполняются условия:

1) для каждого $h \in \mathfrak{H}$ функция $\left\|\mathcal{A}_{0}(x, h) w^{-1}(x)\right\|$ принадлежит $L_{2}(\mathbb{R})$;

2) существует константа $m>0$ такая, что

$$
m\|h\|_{\mathfrak{H}}^{2} \leqslant \int_{\mathbb{R}}\left\|\mathcal{A}_{0}(x, h) w^{-1}(x)\right\|^{2} d x
$$

для всех $h \in \mathfrak{H}$.

Основную задачу о полугруппах $U_{ \pm}(t)$ мы будем решать в классе $w$-возмущений операторов $A_{0}$. Изложим причины, по которым рассмотрение $w$-возмущений представляется естественным.

Во-первых, в случае $n=1$ условие того, что оператор есть $w$-возмущение, фактически является необходимым для принадлежности полугруппы $U_{-}(t)$ классу $C_{0}$. В самом деле, пусть оператор $A$, обратный к которому задается формулой

$$
A^{-1} h=A_{0}^{-1} h+(h, f) g, \quad h \in \mathfrak{H}, \quad A_{0} \in \Sigma^{(\exp )},
$$

генерирует $C_{0}$-полугруппу $U_{-}(t)$, и пусть $\varphi(z):=1-z\left(A_{0}\left(A_{0}-z I\right)^{-1} g, f\right)-$ целая функция, удовлетворяющая условиям

$$
\limsup _{y \rightarrow+\infty} y^{-1} \log |\varphi(i y)|=a, \quad \limsup _{y \rightarrow-\infty}\left|y^{-1}\right| \log |\varphi(i y)|=0,
$$

где через а обозначен экспоненциальный тип резольвенты $\left(A_{0}-z I\right)^{-1}$. Тогда справедливы следующие утверждения:

а) вес $w^{2}(x):=\left\|A_{0}\left(A_{0}-x I\right)^{-1} g\right\|^{2}$ удовлетворяет скалярному $\left(A_{2}\right)$-условию Макенхаупта [8];

b) существуют положительные константы $m, M$ такие, что

$$
m\|h\|_{\mathfrak{H}}^{2} \leqslant \int_{\mathbb{R}}\left|\mathcal{A}_{0}(x, h) w^{-1}(x)\right|^{2} d x \leqslant M\|h\|_{\mathfrak{H}}^{2},
$$


где в полном соответствии с (0.2) имеем

$$
\mathcal{A}_{0}(z, h)=\left(A_{0}\left(A_{0}-z I\right)^{-1} g, h\right), \quad w^{2}(x)=\left\|A_{0}\left(A_{0}-x I\right)^{-1} g\right\|^{2} .
$$

Оба эти результата доказаны в работе [9, теорема 2.1 , предложение 4.2 , теорема 4.4]). Таким образом, в случае одномерного возмущения указан скалярный $\left(A_{2}\right)$-вес $w^{2}$, относительно которого $A$ есть $w$-возмущение ранга 1 .

Во-вторых, в настоящей работе приведены случаи, когда условие того, что оператор есть $w$-возмущение ранга $n$, является необходимым для принадлежности полугруппы $\exp \{-i A t\}$ классу $C_{0}$. Речь идет о плотно заданных операторах $\tilde{A}$ вида

$$
(\tilde{A})^{-1} h=B^{*} h+\sum_{k=1}^{n}\left(h, f_{k}\right) g_{k}, \quad h \in \mathfrak{H},
$$

где $B$ - такой диссипативный оператор с $n$-мерной мнимой частью $\operatorname{Im} B$, что $\sigma(B)=\{0\}$, Ker $B=\{0\}$. Обозначим через $\Theta(z)$ характеристическую матрицу-функцию оператора $B$ (см. $\S 1)$. В статье доказано следующее утверждение (теорема 3.4). Пусть в формуле (0.3) векторы $\left\{f_{k}\right\}_{k=1}^{n}$ образуют базис пространства несамоспряженности $(\operatorname{Im} B) \mathfrak{H}$, и пусть также полугруппа $\exp \{-i \tilde{A} t\}, t \geqslant 0$, принадлежит классу $C_{0}$. Тогда если при некотором $\delta>0$ матрица-функция $e^{-i \delta z} \Theta(z)$ ограничена в верхней полуплоскости, то оператор $\tilde{A}$ является $w$-возмущением ранга $n$ оператора $A_{0}:=\left(B^{*}\right)^{-1}$ класса $\Sigma^{(\exp )}$.

Таким образом, из сформулированных утверждений следует, что при изучении полугруппы $U_{-}(t)$ условие того, что оператор $A$ вида (0.1) является $w$-возмущением оператора $A_{0}$, существенно и от него, вообще говоря, отказаться нельзя.

Формально основными результатами статьи являются теоремы $2.3,3.2$, дающие описание в классе $w$-возмущений генераторов полугрупп $U_{ \pm}(t)$ со свойством $C_{0}$. Вместе с тем в статье доказан ряд вспомогательных результатов, представляющих самостоятельный интерес. В частности, подход к описанию генераторов с помощью интегральных оценок норм резольвент (теорема 2.1) может быть использован для решения и других задач теории возмущения полугрупп, например задачи об описании $C_{0}$-полугрупп $\exp \{ \pm i A t\}$, если в формуле (0.1) считать $A_{0}^{-1}$ произвольным антидиссипативным оператором с конечномерной мнимой частью и дискретным спектром.

Статья состоит из введения и четырех параграфов. В $\S 1$ доказан ряд вспомогательных результатов, которые используются на протяжении всей статьи. Здесь доказывается, что каждое $w$-возмущение $A$, кроме матричного $\left(A_{2}\right)$-веса $w^{2}$, который фигурирует в определении 0.1 , порождает также внутреннюю в области $\operatorname{Im} z>0$ целую матрицу-функцию $\Theta(z)$. Доказывается также (теорема 1.5$)$, что $w$-возмущение $A$ подобно оператору $\tilde{A}$ вида $(0.3)$, в котором оператор $B^{*}$ в модельном пространстве $\mathcal{K}_{\Theta}:=H_{+}^{2}\left(\mathbb{C}^{n}\right) \ominus \Theta H_{+}^{2}\left(\mathbb{C}^{n}\right)$ действует по формуле

$$
\left(B^{*} h\right)(z)=z^{-1}(h(z)-\Theta(z) h(0)), \quad h \in \mathcal{K}_{\Theta},
$$

векторы $g_{k}, 1 \leqslant k \leqslant n$, каноническим образом определяются по весу $w^{2}$. Следовательно, этот результат позволяет свести общую задачу о полугруппах к рассмотрению специальных возмущений модельных операторов.

В $\S 2,3$ доказаны основные теоремы $2.3,3.2$, которые дают описание генераторов $C_{0}$-полугрупп $U_{ \pm}(t)$. Обе теоремы формулируются в терминах внутренней матрицы-функции $\Theta$ и веса $w^{2}$, которые отвечают $w$-возмущению $A$, 
а также в терминах целой матрицы-функции $\Phi(z)$ с элементами

$$
\Phi_{k j}(z)=\delta_{k j}-z\left(A_{0}\left(A_{0}-z I\right)^{-1} g_{j}, f_{k}\right), \quad 1 \leqslant k, j \leqslant n .
$$

В теоремах 2.3, 3.2 необходимые условия совпадают с достаточными, если дополнительно предположить, что при некотором $\delta>0$ матрица-функция $e^{-i z \delta} \Theta(z)$ ограничена в верхней полуплоскости. Мы предполагаем, что это условие в классе всех $w$-возмущений существенно и от него, вообще говоря, отказаться нельзя.

Несмотря на схожесть формулировок теорем $2.3,3.2$, они решают принципиально разные задачи. В теореме 3.2 речь идет о полугруппе $U_{+}(t)$ при наличии полугруппы $V_{+}(t), t \geqslant 0$, а в теореме 2.3 дается критерий существования $U_{-}(t)$, в то время как полугруппы $V_{-}(t):=\exp \left\{-i A_{0} t\right\}$ не существует. Этим обстоятельством объясняется также существенные различия в доказательствах обеих теорем.

В $\S 4$ рассматриваются примеры применений основных теорем работы. В частности, если в качестве $A$ взять оператор дифференцирования в $L_{2}$-пространстве вектор-функции, заданной на отрезке, область определения которого задается некоторой системой функционалов, то приходим к полугруппам сдвигов вдоль специальных продолжений вектор-функций за пределы отрезка. На этом факте основано применение теорем о полугруппах к решению специальных систем интегро-дифференциальных уравнений со сдвигом аргумента.

Ограниченные рамки статьи не позволили рассмотреть задачи спектрального анализа $w$-возмущений, перечень которых приведен, например, в обзоре [10]. Мы ограничились лишь формулировкой следствий из теорем 2.3, 3.2 о подобии диссипативному (антидиссипативному) модельному оператору. Дальнейшее исследование модельных операторов дает возможность получить ответы на ряд естественных вопросов спектральной теории (условия полноты и базисности семейств корневых векторов, методы суммирования спектральных разложений, формулы для экспоненциальных типов полугрупп $U_{ \pm}(t)$ и др. [10]).

Настоящая статья, как и ее краткое изложение [11], посвящается столетию М. Г. Крейна; воспоминания о годах, проведенных рядом с ним, навсегда останутся в нашей памяти.

На протяжении всей работы мы используем следующие обозначения: $\mathbb{C}_{ \pm}-$ верхняя, нижняя полуплоскости; $H_{ \pm}^{2}\left(\mathbb{C}^{n}\right)$ - класс Харди $\mathbb{C}^{n}$-значных вектор-функций (столбцов) в областях $\mathbb{C}_{ \pm} ;{ }^{*} H_{ \pm}^{2}\left(\mathbb{C}^{n}\right)-$ классы Харди $\mathbb{C}^{n}$-значных вектор-функций (строк).

Для неотрицательного почти всюду $(n \times n)$-матричного веса $w^{2}$ через $L_{2}^{n}\left(w^{2}\right)$ обозначается лебегово пространство $\mathbb{C}^{n}$-значных функций (столбцов) $f(x)$ с нормой

$$
\int_{\mathbb{R}}\left(w^{2}(x) f(x), f(x)\right)_{\mathbb{C}^{n}} d x<\infty .
$$

Скалярное произведение в $\mathbb{C}^{n}$ считается евклидовым. В случае $w^{2}(x) \equiv E_{n}$ (единичная матрица) используются обозначения $L_{2}^{n}(\mathbb{R})$ и $L_{2}^{n}(0, a)$, если векторфункции рассматриваются на $[0, a]$. Аналогичные пространства $\mathbb{C}^{n}$-значных вектор-функций (строк) обозначаются через ${ }^{*} L_{2}^{n}\left(w^{2}\right),{ }^{*} L_{2}^{n}(\mathbb{R}),{ }^{*} L_{2}^{n}(0, a)$.

Для ограниченного оператора $B$ обозначим $\operatorname{Im} B=\left(B-B^{*}\right) /(2 i)$. Оператор $B$ называется диссипативным (антидиссипативным), если $\operatorname{Im} B \geqslant 0$ $(\operatorname{Im} B \leqslant 0)$.

Через ${ }^{t} A$ обозначается транспонированная матрица $A$. 


\section{§1. Вспомогательные результаты и редукция основной задачи}

1.1. В этом пункте мы изложим ряд фактов о матричных $\left(A_{2}\right)$-весах Макенхаупта, которые будут использоваться на протяжении всей статьи.

В дальнейшем произвольный $(n \times n)$-матричный вес на вещественной оси удобно обозначать через $w^{2}$.

Матричное условие $\left(A_{2}\right)$ состоит в том, что [12]

$$
\sup _{\Delta}\left\{\left\|\left(M w^{2}\right)^{1 / 2}\left(M w^{-2}\right)^{1 / 2}\right\|\right\}<\infty,
$$

где

$$
M w^{ \pm 2}:=\frac{1}{|\Delta|} \int_{\Delta} w^{ \pm 2}(x) d x,
$$

$\Delta$ - произвольный интервал вещественной оси, $|\Delta|-$ его длина.

Из условия $\left(A_{2}\right)$ легко выводится, что

$$
\int_{\mathbb{R}} \frac{\left(\operatorname{det} w^{2}(x)\right)^{ \pm 1 / n}}{1+x^{2}} d x<\infty .
$$

Этот факт лежит в основе следующей теоремы о факторизации $\left(A_{2}\right)$-весов Макенхаупта.

Теорема A [12], [13]. Пусть $w^{2}$ - произвольный матричный $\left(A_{2}\right)$-вес на $\mathbb{R}$. Тогда существует аналитическая в области $\mathbb{C}_{+}\left(\right.$соответственно, $\left.\mathbb{C}_{-}\right)$матрица-функиия $w_{+}\left(\right.$соответственно, $\left.w_{-}\right)$, имеющая почти всюду на $\mathbb{R}$ некасательные граничные значения $w_{+}(x-i 0)$ (соответственно, $\left.w_{-}(x-i 0)\right)$ такие, что выполняются следующие условия:

1) имеют место факторизации

$$
w^{2}(x) \stackrel{n . \text { в. }}{=} w_{+}^{*}(x+i 0) w_{+}(x+i 0) \stackrel{n . \text {. }}{=} w_{-}^{*}(x-i 0) w_{-}(x-i 0), \quad x \in \mathbb{R} ;
$$

2) функция $\operatorname{det} w_{+}(z)\left(\operatorname{det} w_{-}(z)\right)$ является внешней $[8]$ в $\mathbb{C}_{+}\left(\mathbb{C}_{-}\right)$;

3) элементы матриц-фуункиий $w_{+}(z)(z+i)^{-1}$ u $w_{-}(z)(z-i)^{-1}$ принадлежат классам Харди $\mathrm{H}_{+}^{2}$ и $\mathrm{H}_{-}^{2}$ соответственно;

4) обе факторизачии из (1.1) единственны с точностъю до постоянных левых унитарных множителей.

Заметим, что вес Макенхаупта $w^{2}(x)$ допускает также факторизацию вида

$$
w^{2}(x) \stackrel{\text { п.в. }}{=} \stackrel{\circ}{w}_{+}(x+i 0) \stackrel{\circ}{w}_{+}^{*}(x+i 0) \stackrel{\text { п.в. }}{=} \stackrel{\mathfrak{w}_{-}}{ }(x-i 0) \stackrel{\circ}{w}_{-}^{*}(x-i 0),
$$

где матрицы-функции $\stackrel{\circ}{+}_{+}, \stackrel{\circ}{\mathfrak{w}}_{-}$обладают аналогичными свойствами. В частности, матрицы-функции $\stackrel{\circ}{+}_{+}, \stackrel{\mathfrak{w}}{-}_{-}$определяются с точностью до правых постоянных унитарных множителей.

Как и в скалярном случае, матричные веса Макенхаупта связаны со свойствами преобразования Гильберта в весовых пространствах. Напомним, что в пространствах вектор-функций преобразование Гильберта действует покоординатно.

Теорема В [12]. Преобразование Гилъберта

$$
(\mathcal{H} f)(x):=\frac{1}{\pi i} f_{\mathbb{R}} \frac{f(t)}{t-x} d t, \quad f(t)=\operatorname{col}\left(f_{k}(t)\right)_{k=1}^{n},
$$

ограничено в пространстве $L_{2}^{n}\left(w^{2}\right)$ тогда и только тогда, когда матричный вес $w^{2}$ удовлетворяет условию $\left(A_{2}\right)$ на $\mathbb{R}$. 
Сформулированные результаты позволяют ввести весовые классы Харди в областях $\mathbb{C}_{ \pm}$. Пусть $w^{2}$ - произвольный матричный $\left(A_{2}\right)$-вес Макенхаупта на $\mathbb{R}, H_{ \pm}^{2}\left(\mathbb{C}^{n}\right)$ - векторные классы Харди. Класс Харди $H_{+}^{2}\left(\mathbb{C}^{n}, w^{2}\right)$ состоит из вектор-функций $F$ (столбцов) таких, что $w_{+}(z) F(z) \in H_{+}^{2}\left(\mathbb{C}^{n}\right)$, причем норма определяется формулой

$$
{ }^{w}\|F\|_{+}^{2}:=\sup _{y>0} \int_{\mathbb{R}}\left\|w_{+}(x+i y) F(x+i y)\right\|_{\mathbb{C}^{n}}^{2} d x .
$$

Аналогично, весовой класс Харди $H_{-}^{2}\left(\mathbb{C}^{n}, w^{2}\right)$ состоит из вектор-функций $F$ таких, что $w_{-}(z) F(z) \in H_{-}^{2}\left(\mathbb{C}^{n}\right)$, с нормой

$$
{ }^{w}\|F\|_{-}^{2}:=\sup _{y<0} \int_{\mathbb{R}}\left\|w_{-}(x+i y) F(x+i y)\right\|_{\mathbb{C}^{n}}^{2} d x .
$$

Отметим, что в последних формулах $w_{ \pm}$- матрицы-функции, входящие в факторизации (1.1) веса $w^{2}$.

Факторизации (1.2) также порождают весовые классы Харди вектор-функций (строк). Вектор-функция $F=\operatorname{row}\left(F_{k}\right)_{k=1}^{n}$ принадлежит классу Харди ${ }^{*} H_{ \pm}^{2}\left(\mathbb{C}^{n}, w^{2}\right)$ тогда и только тогда, когда

$$
F(z) \stackrel{\circ}{w}_{ \pm}(z) \in{ }^{*} H_{ \pm}^{2}\left(\mathbb{C}^{n}\right) .
$$

Кроме того, нормы в этих классах также вводятся формулами

$$
{ }^{w}\|F\|_{ \pm}^{2}:=\left\|F(z) \stackrel{\circ}{w}_{ \pm}(z)\right\|_{ \pm}^{2},
$$

где $\|\cdot\|_{ \pm}^{2}-$ стандартная норма в классах Харди ${ }^{*} H_{ \pm}^{2}\left(\mathbb{C}^{n}\right)$.

В следующей теореме мы сформулируем свойства весовых классов Харди. Все перечисленные в этом пункте результаты либо содержатся в [12], либо несложно выводятся из теоремы В.

Теорема С. Пусть $w^{2}$ - произволъный матричный $\left(A_{2}\right)$-вес на $\mathbb{R}$. Тогда справедливы следующие утверждения:

1) каждая вектор-функиия $F \in H_{+}^{2}\left(\mathbb{C}^{n}, w^{2}\right)$ является аналитической в $\mathbb{C}_{+}$, имеет почти всюду некасательные предельные значения $F(x+i 0)$, принадлежащие пространству $L_{2}^{n}\left(w^{2}\right)$;

2) для каждой функиии $f \in L_{2}^{n}\left(w^{2}\right)$ интеграл Коши

$$
F(z):=\frac{1}{2 \pi i} \int_{\mathbb{R}} \frac{f(x)}{x-z} d x, \quad z \in \mathbb{C}_{+},
$$

принадлежит $H^{2}\left(\mathbb{C}^{n}, w^{2}\right)$ и справедлива оценка

$$
{ }^{w}\|F\|_{+}^{2} \leqslant C \int_{\mathbb{R}}\left(w^{2}(x) f(x), f(x)\right)_{\mathbb{C}^{n}} d x
$$

c некоторой константой $C$;

3) каждая функиия $F \in H_{+}^{2}\left(\mathbb{C}^{n}, w^{2}\right)$ связана со свочми граничными значениями $F(x+i 0)$ формулой

$$
F(z)=\frac{1}{2 \pi i} \int_{\mathbb{R}} \frac{F(x+i 0)}{x-z} d x, \quad z \in \mathbb{C}_{+} ;
$$


4) пространства $H_{ \pm}^{2}\left(\mathbb{C}^{n}, w^{2}\right)$ отождествляются с подпространствами в $L_{2}^{n}\left(w^{2}\right)$, причем существуют ограниченные проекторы

$$
\mathcal{P}_{ \pm} f:=\frac{1}{2}(f \pm \mathcal{H} f)
$$

и в соответствии с этим имеет место прямое разложение

$$
L_{2}^{n}\left(w^{2}\right)=H_{+}^{2}\left(\mathbb{C}^{n}, w^{2}\right) \dot{+} H_{-}^{2}\left(\mathbb{C}^{n}, w^{2}\right) .
$$

Ясно, что справедливы аналоги теорем А-C для пространств вектор-функций (строк) ${ }^{*} L_{2}^{n}\left(w^{2}\right),{ }^{*} H_{ \pm}^{2}\left(\mathbb{C}^{n}, w^{2}\right)$.

1.2. Напомним, что целая $(n \times n)$-матрица-функция $\Theta$ называется внутренней в $\mathbb{C}_{+}$, если выполняются условия

$$
\Theta(z) \Theta^{*}(z)-E_{n} \leqslant 0, \quad z \in \mathbb{C}_{+}, \quad \Theta(x) \Theta^{*}(x)-E_{n}=0, \quad x \in \mathbb{R},
$$

где $E_{n}-$ единичная матрица. Далее рассматриваются иистые внутренние матрицы $\Theta$, т. е. такие, что $\|\Theta(i) c\|<\|c\|$ для всех $c \in \mathbb{C}^{n}$. Кроме того, будем считать, что выполняется условие нормировки $\Theta(0)=E_{n}$. Рассмотрим в модельном пространстве целых вектор-функций (столбцов)

$$
\mathcal{K}_{\Theta}:=H_{+}^{2}\left(\mathbb{C}^{n}\right) \ominus \Theta H_{+}^{2}\left(\mathbb{C}^{n}\right)
$$

ограниченный оператор

$$
(B h)(z)=z^{-1}(h(z)-h(0)), \quad h \in \mathcal{K}_{\Theta} .
$$

Нетрудно проверить, что

$$
\left(B^{*} h\right)(z)=z^{-1}(h(z)-\Theta(z) h(0)), \quad h \in \mathcal{K}_{\Theta} .
$$

Оператор $B$ обладает следующими свойствами:

1) $\sigma(B)=\{0\}$, Ker $B=\{0\}$

2) $\operatorname{dim}(\operatorname{Im} B) \mathcal{K}_{\Theta}=n$;

3) $\operatorname{Im} B \geqslant 0$ (диссипативность).

Сформулируем теперь основной результат о функциональной модели несамосопряженного оператора, который обладает свойствами 1)-3). Пусть оператор $\stackrel{B}{B}$, действующий в сепарабельном гильбертовом пространстве $\mathfrak{H}$, диссипативен и имеет $n$-мерную мнимую часть:

$$
\frac{1}{i}\left(\stackrel{\circ}{B}-\stackrel{\circ}{B}^{*}\right) h=\sum_{k=1}^{n}\left(h, \varphi_{k}\right) \varphi_{k}, \quad h \in \mathfrak{H} .
$$

Матрица-функция $\Theta$, элементы которой определяются формулой

$$
\Theta_{j k}(z)=\delta_{k j}+i z\left((I-z \stackrel{\circ}{B})^{-1} \varphi_{k}, \varphi_{j}\right), \quad 1 \leqslant k, j \leqslant n,
$$

называется характеристической матрищей-функиией оператора $\stackrel{\circ}{B}$. Если

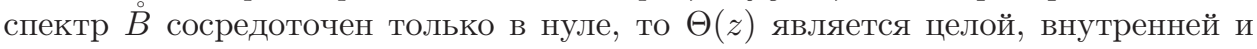
чистой.

TeOpema D. Пусть $\stackrel{\circ}{B}$ - произвольный диссипативный оператор со спектром в нуле, тривиальным ядром и п-мерной мнимой частью, действующий в гилъбертовом пространстве $\mathfrak{H}$. Тогда $B$ унитарно эквивалентен моделъному оператору $B$ вида (1.3) в пространстве $\mathcal{K}_{\Theta}$, где $\Theta$ - характеристическая матрица-функция оператора $\stackrel{\circ}{B}$.

Все перечисленные в этом пункте факты можно найти в [12]-[15] (в [14] излагается аналог теории для сжатий). 
1.3. Через $\mathcal{M}_{n}^{2}$ в дальнейшем обозначается множество $(n \times n)$-матричных $\left(A_{2}\right)$-весов Макенхаупта на $\mathbb{R}$. Далее, множество всех целых внутренних в $\mathbb{C}_{+}$ чистых матриц-функций $\Theta$, удовлетворяющих условию $\Theta(0)=E_{n}$, обозначим через $J_{n}$. Условимся также, что $\mathbb{P}_{\Theta}$ - ортопроектор из $H_{+}^{2}\left(\mathbb{C}^{n}\right)$ на модельное подпространство $\mathcal{K}_{\Theta}$.

Пусть $\Theta \in J_{n}, w^{2} \in \mathcal{M}_{n}^{2}$. В пространстве $\mathcal{K}_{\Theta}$ введем векторы $g_{k}^{\Theta}, 1 \leqslant k \leqslant n$, с помощью формул

$$
\left(I+i B^{*}\right)^{-1} g_{k}^{\Theta}=\mathbb{P}_{\Theta} \frac{w_{+}^{k}(x+i 0)}{x+i},
$$

где $B^{*}$ определяется формулой $(1.4), w_{+}-$матрица-функция из факторизации $(1.1), w_{+}^{k}(z)-k$-й столбец матрицы $w_{+}(z), z \in \mathbb{C}_{+}$. Определение векторов $g_{k}^{\Theta}$ корректно, поскольку $w_{+}^{k}(z)(z+i)^{-1} \in H_{+}^{2}\left(\mathbb{C}^{n}\right)$ (теорема A).

Лемма 1.1. Для всех $z \in \mathbb{C}_{+}$имеют место формуль

$$
\left(I+z B^{*}\right)^{-1} g_{k}^{\Theta}=\mathbb{P}_{\Theta} \frac{w_{+}^{k}(x+i 0)}{x+z}, \quad 1 \leqslant k \leqslant n .
$$

ДокАЗАТЕльство. Из формулы (1.4) легко выводится формула для резольвенты:

$$
\left(I+z B^{*}\right)^{-1} g_{k}^{\Theta}=\frac{\lambda g^{\Theta}(\lambda)+z \Theta(\lambda) \Theta^{-1}(-z) g^{\Theta}(-z)}{z+\lambda}, \quad g^{\Theta} \in \mathcal{K}_{\Theta} .
$$

Поскольку

$$
\left(I+z B^{*}\right)^{-1}\left(I+i B^{*}\right) h=z^{-1}\left((z-i)\left(I+z B^{*}\right)^{-1} h+i h\right),
$$

с учетом (1.7) получим

$$
\left(I+z B^{*}\right)^{-1}\left(I+i B^{*}\right) h=\frac{\lambda+i}{\lambda+z} h(\lambda)+\frac{z-i}{\lambda+z} \Theta(\lambda) \Theta^{-1}(-z) h(-z) .
$$

Полагая здесь $h=\mathbb{P}_{\Theta} u_{k}$, где $u_{k}(\lambda)=w_{+}^{k}(\lambda)(\lambda+i)^{-1}$, и учитывая (1.6), найдем

$$
\left(I+z B^{*}\right)^{-1} g_{k}^{\Theta}=\frac{\lambda+i}{\lambda+z} \mathbb{P}_{\Theta} u_{k}+\frac{z-i}{\lambda+z} \Theta(\lambda) \Theta^{-1}(-z)\left(\mathbb{P}_{\Theta} u_{k}\right)(-z), \quad \lambda \in \mathbb{C}_{+} .
$$

Заметим, что имеют место представления $\mathbb{P}_{\Theta} u_{k}-u_{k}=\Theta(\lambda) h_{k}(\lambda)$, где $h_{k} \in$ $H_{+}^{2}\left(\mathbb{C}^{n}\right)$. Поэтому приходим к равенствам

$$
\left(I+z B^{*}\right)^{-1} g_{k}^{\Theta}=\frac{\lambda+i}{\lambda+z} u_{k}(\lambda)+\Theta(\lambda)\left(\frac{\lambda+i}{\lambda+z} h_{k}(\lambda)+\frac{z-i}{\lambda+z} \Theta^{-1}(-z)\left(\mathbb{P}_{\Theta} u_{k}\right)(-z)\right),
$$

из которых вытекает утверждение леммы:

$$
\left(I+z B^{*}\right)^{-1} g_{k}^{\Theta}=\mathbb{P}_{\Theta} \frac{x+i}{x+z} u_{k}(x+i 0)=\mathbb{P}_{\Theta} \frac{w_{+}^{k}(x+i 0)}{x+z}, \quad 1 \leqslant k \leqslant n, \quad z \in \mathbb{C}_{+} .
$$

По матрице-функции $\Theta \in J_{n}$ и весу $w^{2} \in M_{n}^{2}$ в модельном пространстве $\mathcal{K}_{\Theta}$ построим отображение

$$
\mathcal{B}(z, h):=-\frac{1}{2 \pi i} \operatorname{row}\left(\left(I-z B^{*}\right)^{-1} g_{k}^{\Theta}, h\right)_{k=1}^{n},
$$

где оператор $B^{*}$ и векторы $g_{k}^{\Theta}, 1 \leqslant k \leqslant n$, имеют прежний смысл. Заметим, что это отображение каждой целой вектор-функции $h \in \mathcal{K}_{\Theta}$ ставит в соответствие целую вектор-функцию $\mathcal{B}(z, h)$. 
ТеОрема 1.1. Пусть $\mathcal{B}(z, h)$ - преобразование вида (1.8), порожденное парой $\Theta \in J_{n}, w^{2} \in \mathcal{M}_{n}$. Тогда существует такая константа $M$, что для всех вектор-функций $h \in \mathcal{K}_{\Theta}$ выполнено

$$
\|h\|_{\mathcal{K}_{\Theta}}^{2} \leqslant \int_{\mathbb{R}}\left(\mathcal{B}(x, h) w^{-2}(x), \mathcal{B}(x, h)\right)_{\mathbb{C}^{n}} d x \leqslant M\|h\|_{\mathcal{K}_{\Theta}}^{2} .
$$

ДокАзАтЕльство. Для произвольного вектора $h(\lambda)=\operatorname{col}\left(h_{k}(\lambda)\right)_{k=1}^{n} \in \mathcal{K}_{\Theta}$ из леммы 1.1 следует, что для любого $z \in \mathbb{C}_{-}$

$$
\begin{aligned}
-\frac{1}{2 \pi i}\left(\left(I-z B^{*}\right)^{-1} g_{k}^{\Theta}, h\right) & =-\frac{1}{2 \pi i}\left(\mathbb{P}_{\Theta} \frac{w_{+}^{k}(x+i 0)}{x-z}, h\right)_{\mathcal{K}_{\Theta}} \\
& =-\frac{1}{2 \pi i} \int_{\mathbb{R}} \frac{\sum_{j=1}^{n} w_{+}^{j k}(x+i 0) \overline{h_{j}(x)}}{x-z} d x .
\end{aligned}
$$

Таким образом,

$$
\mathcal{B}(z, h)=-\frac{1}{2 \pi i} \int_{\mathbb{R}} \frac{h^{*}(x) w_{+}(x+i 0)}{x-z} d x, \quad z \in \mathbb{C}_{-},
$$

где введено обозначение $h^{*}(z)=\operatorname{row}\left(\overline{h_{k}(\bar{z})}\right)_{k=1}^{n}$. Заметим, что если $w^{2} \in \mathcal{M}_{n}^{2}$, то $w^{-2} \in \mathcal{M}_{n}^{2}$. Кроме того, функция $h^{*}(x) w_{+}(x+i 0)$ принадлежит пространству строк ${ }^{*} L_{2}^{n}\left(w^{-2}\right)$. В силу аналога теоремы С для строк проектор $\mathcal{P}_{-}$из ${ }^{*} L_{2}\left(w^{-2}\right)$ на ${ }^{*} H_{+}^{2}\left(\mathbb{C}^{n}, w^{-2}\right)$ записываются в виде $\mathcal{P}_{-}=\frac{1}{2}(I-\mathcal{H})$. Поэтому, устремляя $z \mathrm{~K}$ $u \in \mathbb{R}$ некасательно в (1.9), получим

$$
\mathcal{B}(u, h)=\frac{1}{2} h^{*}(u) w_{+}(u+i 0)-\frac{1}{2 \pi i} f_{\mathbb{R}} \frac{h^{*}(x) w_{+}(x+i 0)}{x-u} d x
$$

для каждого $h \in \mathcal{K}_{\Theta}$. Следовательно,

$$
\mathcal{B}(z, h) w_{+}^{-1}(u+i 0)=\frac{1}{2} h^{*}(u)-\frac{1}{2 \pi i} f_{\mathbb{R}} \frac{h^{*}(x) w_{+}(x+i 0)}{x-u} d x w_{+}^{-1}(u+i 0) .
$$

Поскольку $w^{2}(x) \stackrel{\text { п.в. }}{=} w_{+}^{*}(x+i 0) w_{+}(x+i 0)$, из теоремы В легко вытекает ограниченность взвешенного преобразования Гильберта:

$$
\int_{\mathbb{R}}\left\|\mathcal{H}\left(h^{*}(x) w_{+}(x+i 0)\right) w_{+}^{-1}(u+i 0)\right\|_{\mathbb{C}^{n}}^{2} d u \leqslant M_{1} \int_{\mathbb{R}}\left\|h^{*}(u)\right\|_{\mathbb{C}^{n}}^{2} d u .
$$

Поэтому из равенства (1.10) следует оценка

$$
\begin{aligned}
\int_{\mathbb{R}}\left\|\mathcal{B}(u, h) w_{+}^{-1}(u+i 0)\right\|_{\mathbb{C}^{n}}^{2} d u & \leqslant M \int_{\mathbb{R}}\left\|h^{*}(x)\right\|_{\mathbb{C}^{n}}^{2} d x=M \int_{\mathbb{R}}\|h(x)\|_{\mathbb{C}^{n}}^{2} d x \\
& =M\|h\|_{\mathcal{K}_{\Theta}}^{2} .
\end{aligned}
$$

Перепишем теперь $(1.10)$ с учетом равенства $\frac{1}{2} \mathcal{H}=\mathcal{P}_{+}-\frac{1}{2} I$, где $\mathcal{P}_{+}-$проектор из ${ }^{*} L_{2}^{n}\left(w^{-2}\right)$ на ${ }^{*} H_{+}^{2}\left(\mathbb{C}^{n}, w^{-2}\right)$. Мы получим равенство

$$
\mathcal{B}(u, h) w_{+}^{-1}(u+i 0)=h^{*}(u)-\left(\mathcal{P}_{+} h^{*}(x) w_{+}(x+i 0)\right) w_{+}^{-1}(u+i 0),
$$

слагаемые правой части которого ортогональны. Действительно, поскольку $h \in H_{+}^{2}\left(\mathbb{C}^{n}\right)$, то $h^{*} \in{ }^{*} H_{-}^{2}\left(\mathbb{C}^{n}\right)$. Кроме того, из аналога теоремы С для строк следует, что

$$
\left(\mathcal{P}_{+} h^{*} w_{+}(x+i 0)\right) w_{+}^{-1}(u+i 0) \in{ }^{*} H_{+}^{2}\left(\mathbb{C}^{n}\right) .
$$

Таким образом, справедлива следующая оценка снизу:

$$
\int_{\mathbb{R}}\left\|\mathcal{B}(u, h) w_{+}^{-1}(u+i 0)\right\|_{\mathbb{C}^{n}}^{2} d u \geqslant \int_{\mathbb{R}}\left\|h^{*}(u)\right\|_{\mathbb{C}^{n}}^{2} d u=\|h\|_{\mathcal{K}_{\Theta}}^{2},
$$

тем самым теорема доказана. 
В простейшем случае

$$
\Theta(z)=e^{i a z} E_{n}, \quad a>0, \quad w^{2}(x) \equiv E_{n}, \quad x \in \mathbb{R},
$$

модельное пространство $\mathcal{K}_{\Theta}=H_{+}^{2}\left(\mathbb{C}^{n}\right) \ominus e^{i a z} H_{+}^{2}\left(\mathbb{C}^{n}\right)$ в силу теоремы ВинераПэли [8] совпадает с множеством функций вида

$$
h(z)=\int_{0}^{a} e^{i z t} f(t) d t, \quad f=\operatorname{col}\left(f_{k}\right)_{k=1}^{n}, \quad f \in L_{2}^{n}(0, a) .
$$

Поскольку $w_{+}(x+i 0) \equiv E_{n}, x \in \mathbb{R}$, из формулы (1.9) следует, что

$$
\mathcal{B}(z, h)=\int_{0}^{a} e^{-i z t} f^{*}(t) d t=h^{*}(z), \quad z \in \mathbb{C} .
$$

Таким образом, в этом простейшем случае отображение $\mathcal{B}(x, h)$ является изометрией.

Следующая теорема описывает образ преобразования (1.8).

Теорема 1.2. Целая вектор-функиия $\mathcal{F}(z):=\operatorname{row}\left(f_{k}(z)\right)_{k=1}^{n}$ тогда и только тогда представима в виде 1

$$
\mathcal{F}(z)=\mathcal{B}(z, h)
$$

с некоторой функиией $h \in \mathcal{K}_{\Theta}$, когда $\mathcal{F}$ удовлетворяет следующим условиям:

1) $\mathcal{F}(x) w_{+}^{-1}(x+i 0) \Theta(x) \in{ }^{*} H_{+}^{2}\left(\mathbb{C}^{n}\right)$;

2) $\mathcal{F}(x) w_{-}^{-1}(x+i 0) \in{ }^{*} H_{-}^{2}\left(\mathbb{C}^{n}\right)$.

ДокАзАтЕльство. Пусть $\mathcal{F}$ допускает представление (1.12). Тогда из формулы (1.9) следует, что

$$
\mathcal{F}(x)=\mathcal{P}_{-}\left(h^{*}(x) w_{+}(x+i 0)\right),
$$

т. е. $\mathcal{F} \in{ }^{*} H_{-}^{2}\left(\mathbb{C}^{n}, w^{-2}\right)$. Если учесть, что равенство

$$
w^{-2}(x) \stackrel{\text { п. в. }}{=} w_{-}^{-1}(x-i 0)\left(w_{-}^{-1}(x-i 0)\right)^{*}
$$

является факторизацией вида (1.2), то из определения весовых классов Харди (см. п. 1.1) следует условие 2). Далее, поскольку $h \in \mathcal{K}_{\Theta}$, для любой функции $g \in H_{+}^{2}\left(\mathbb{C}^{n}\right)$ имеем

$$
0=(h, \Theta g)=\left(\Theta^{*} h, g\right),
$$

т. е. $\Theta^{*}(\bar{z}) h(z) \in H_{-}^{2}\left(\mathbb{C}^{n}\right)$, и поэтому $h^{*}(z) \Theta(z) \in{ }^{*} H_{+}^{2}\left(\mathbb{C}^{n}\right)$. Умножая теперь равенство (1.11) на $\Theta(u)$ справа, заключаем, что выполняется условие 1) доказываемой теоремы.

Обратно, пусть выполнены условия 1), 2) теоремы. Поскольку

$$
\int_{\mathbb{R}}\left\|\mathcal{F}(x) w_{+}^{-1}(x+i 0)\right\|_{\mathbb{C}^{n}}^{2} d x<\infty
$$

имеет место ортогональное разложение

$$
\mathcal{F}(x) w_{+}^{-1}(x+i 0)=f_{-}(x)+f_{+}(x), \quad f_{ \pm} \in{ }^{*} H_{ \pm}^{2}\left(\mathbb{C}^{n}\right) .
$$

\footnotetext{
${ }^{1}$ Из теоремы 1.1 следует, что такое представление единственно.
} 
Вместе с условием 1) это означает, что $f_{-} \Theta \in{ }^{*} H_{+}^{2}\left(\mathbb{C}^{n}\right)$ и, следовательно, $\Theta^{*}(\bar{z}) f_{-}^{*}(z) \in H_{-}^{2}\left(\mathbb{C}^{n}\right)$, где $f_{-}^{*}(z):=\operatorname{col}\left(\overline{f_{-}^{k}(\bar{z})}\right)_{k=1}^{n}$, если $f_{-}(z)=\operatorname{row}\left(f_{-}^{k}(z)\right)_{k=1}^{n}$. Поэтому $h:=f_{-}^{*} \in \mathcal{K}_{\Theta}$, и можем рассмотреть разность

$$
G(z):=\mathcal{F}(z)-\mathcal{B}(z, h), \quad h=f_{-}^{*} .
$$

Далее, во-первых, из (1.13) следует, что

$$
\mathcal{F}(x) w_{+}^{-1}(x+i 0)=h^{*}(x)+f_{+}(x),
$$

во-вторых, из (1.11) выводим равенство

$$
\mathcal{B}(x, h) w_{+}^{-1}(x+i 0)=h^{*}(x)+\tilde{f}_{+}(x),
$$

где $\tilde{f}_{+}-$некоторая функция из ${ }^{*} H_{+}^{2}\left(\mathbb{C}^{n}\right)$. Из двух последних равенств вытекает, что $G(x) w_{+}^{-1}(x+i 0) \in{ }^{*} H_{+}^{2}\left(\mathbb{C}^{n}\right)$, т. е. $G \in{ }^{*} H_{+}^{2}\left(\mathbb{C}^{n}, w^{-2}\right)$. С другой стороны, из условия 2$)$, формулы (1.9) и равенства

$$
G(x) w_{-}^{-1}(x-i 0)=\mathcal{F}(x) w_{-}^{-1}(x-i 0)-\mathcal{B}(x, h) w_{-}^{-1}(x-i 0)
$$

следует, что $G(x) w_{-}^{-1}(x-i 0) \in{ }^{*} H_{-}^{2}\left(\mathbb{C}^{n}\right)$, т. е. $G \in{ }^{*} H_{-}^{2}\left(\mathbb{C}^{n}, w^{-2}\right)$. Таким образом, $G=0$, что и доказывает теорему.

Далее модельное пространство в случае $\Theta(z)=e^{i a z} E_{n}, a>0$, будем обозначать через $\mathcal{K}_{a}$, т. е.

$$
\mathcal{K}_{a}=H_{+}^{2}\left(\mathbb{C}^{n}\right) \ominus e^{i a z} H_{+}^{2}\left(\mathbb{C}^{n}\right) .
$$

Если $\Theta \in J_{n}$, то целая матрица-функция $\Theta$ имеет экспоненциальный тип роста. Этот факт легко вытекает, например, из теоремы Потапова [16], в силу которой $\Theta$ допускает представление в виде мультипликативного интеграла.

Для функции $\Theta \in J_{n}$ обозначим через $a>0$ ее экспоненциальный тип. Тогда имеет место включение

$$
\mathcal{K}_{\Theta} \subseteq \mathcal{K}_{a}
$$

из которого вытекает, что элементы произвольного пространства $\mathcal{K}_{\Theta}$ суть целые вектор-функции экспоненциального типа. Более того, из теоремы Винера-Пэли следует, что каждая функция $h \in \mathcal{K}_{\Theta}$ изображается интегралом Фурье-Лапласа:

$$
h(z)=\int_{0}^{a} e^{i z t} f(t) d t, \quad f=\operatorname{col}\left(f_{k}\right)_{k=1}^{n}, \quad f_{k} \in L_{2}(0, a) .
$$

Заметим, что включение (1.14) есть простое следствие утверждения [17]: если $a$ - экспоненциальный тип матрицы-функции $\Theta \in J_{n}$, то существует $\widetilde{\Theta} \in J_{n}$ такая, что

$$
\Theta(z) \widetilde{\Theta}(z)=e^{i a z} E_{n} .
$$

Теперь мы можем уточнить теорему 1.2 в случае $\Theta(z)=e^{i a z} E_{n}, a>0$. Условимся обозначать оператор $B^{*}$ в $\mathcal{K}_{a}$ через $B_{a}^{*}$ (см. (1.4)). Векторы $g_{k}^{\Theta}$, определенные равенствами (1.6) в пространстве $\mathcal{K}_{a}$, обозначим через $g_{k}^{a}, 1 \leqslant$ $k \leqslant n$. Итак, мы приведем другое описание образа преобразования

$$
\mathcal{B}_{a}(z, h):=-\frac{1}{2 \pi i} \operatorname{row}\left(\left(I-z B_{a}^{*}\right)^{-1} g_{k}^{a}, h\right), \quad h \in \mathcal{K}_{a} .
$$

Напомним, что индикатор роста целой функции $f$ экспоненциального типа определяется формулой

$$
h(\alpha ; f):=\limsup _{r \rightarrow \infty} r^{-1} \log \left|f\left(r e^{i \alpha}\right)\right|, \quad-\pi<\alpha \leqslant \pi .
$$


ТЕорема 1.3. Целая вектор-функиия $\mathcal{F}=\operatorname{row}\left(f_{k}(z)\right)_{k=1}^{n}$ экспоненииального типа тогда и только тогда допускает представление

$$
\mathcal{F}(z)=\mathcal{B}_{a}(z, h)
$$

с некоторой функиией $h \in \mathcal{K}_{a}$, когда выполняются следующие условия:

1) $\int_{\mathbb{R}}\left(\mathcal{F}(x) w^{-2}(x), \mathcal{F}(x)\right)_{\mathbb{C}^{n}} d x<\infty$;

2) $h\left(\frac{\pi}{2}, f_{k}\right) \leqslant a, h\left(-\frac{\pi}{2}, f_{k}\right) \leqslant 0$ для всех $k=1,2, \ldots, n$.

ДокАЗАтЕЛьСтво. Пусть $\mathcal{F}(z)=\mathcal{B}_{a}(z, h)$. Тогда из факторизации (1.1) и условия 2) теоремы 1.2 выводим, что

$$
\int_{\mathbb{R}}\left\|\mathcal{F}(x) w_{-}^{-1}(x-i 0)\right\|_{\mathbb{C}^{n}}^{2} d x=\int_{\mathbb{R}}\left(\mathcal{F}(x) w^{-2}(x), \mathcal{F}(x)\right)_{\mathbb{C}^{n}} d x<\infty .
$$

Далее, рассмотрим в $L_{2}(0, a)$ оператор интегрирования $(J h)(t)=i \int_{0}^{t} h(s) d s$ и введем обозначение

$$
\hat{h}(z)=\frac{1}{\sqrt{2 \pi}} \int_{0}^{a} e^{i z t} h(t) d t, \quad h \in L_{2}(0, a) .
$$

Простое преобразование показывает, что

$$
\widehat{J h}(z)=-\left(\hat{h}(z)-e^{i z t} \hat{h}(0)\right) z^{-1} .
$$

Поэтому в силу (1.4) оператор $B_{a}^{*}$ унитарно эквивалентен оператору

$$
(-J h)(t)=\operatorname{col}\left(-J h_{k}\right)_{k=1}^{n}, \quad h=\operatorname{col}\left(h_{k}\right)_{k=1}^{n},
$$

в пространстве вектор-функций $L_{2}^{n}(0, a)$. Отсюда уже легко выводится условие 2) для индикаторов координат $f_{k}$ вектор-функции $\mathcal{F}_{k}$.

Обратно, пусть выполняются условия 1), 2) теоремы. Из теоремы 1.2 следует, что достаточно установить включения

$$
\mathcal{F}(x) w_{+}^{-1}(x+i 0) e^{i a x} \in{ }^{*} H_{+}^{2}\left(\mathbb{C}^{n}\right), \quad \mathcal{F}(x) w_{-}^{-1}(x-i 0) \in{ }^{*} H_{-}^{2}\left(\mathbb{C}^{n}\right) .
$$

При каждом $\lambda \in \mathbb{C}_{-}$оценим $l_{1}$-норму вектора $\mathcal{F}(x)(x-\lambda)^{-1}$ :

$$
\left\|\frac{\mathcal{F}(x)}{x-\lambda}\right\|_{1}=\left\|\frac{\mathcal{F}(x) w^{-1}(x) w(x)}{x-\lambda}\right\|_{1}=|x-\lambda|^{-1} \sum_{k=1}^{n}\left|\sum_{j=1}^{n} g_{j}(x) w_{j k}(x)\right|,
$$

где $g_{j}(x)$ - координата строки $\mathcal{F}(x) w^{-1}(x)$. Поэтому

$$
\left\|\mathcal{F}(x)(x-\lambda)^{-1}\right\|_{1} \leqslant \sqrt{n}|x-\lambda|^{-1}\left(\sum_{j=1}^{n}\left|g_{j}(x)\right|^{2}\right)^{1 / 2}\left(\sum_{k, j=1}^{n}\left|w_{j k}(x)\right|^{2}\right)^{1 / 2} .
$$

Поскольку $w^{2}$ удовлетворяет матричному условию $\left(A_{2}\right)$, вес $v^{2}(x):=\operatorname{Sp} w^{2}(x)$ является скалярным $\left(A_{2}\right)$-весом Макенхаупта [18]. Поэтому из условия 1) и теоремы А для веса $v^{2}$ следует, что [8]

$$
\begin{aligned}
& \int_{\mathbb{R}}\left\|\mathcal{F}(x)(x-\lambda)^{-1}\right\|_{1} d x \\
& \leqslant \sqrt{n}\left(\int_{\mathbb{R}} \sum_{j=1}^{n}\left|g_{j}(x)\right|^{2} d x\right)^{1 / 2}\left(\int_{\mathbb{R}} v^{2}(x)|x-\lambda|^{-2} d x\right)^{1 / 2}<\infty .
\end{aligned}
$$


В силу условия 2) и элементарных свойств индикатора [19] вытекает, что

$$
\int_{0}^{\infty}\left\|\mathcal{F}(i y)(i y-\lambda)^{-1} e^{i(a+\varepsilon) y}\right\|_{1} d y<\infty, \quad \lambda \in \mathbb{C}_{-},
$$

при каждом $\varepsilon>0$. Таким образом, аналитическая в замыкании $\mathbb{C}_{+}$векторфункция $G_{\varepsilon}(z):=e^{i(a+\varepsilon) z} \mathcal{F}(z)(z-\lambda)^{-1}$ суммируема на сторонах двух углов, образованных вещественной осью и мнимой положительной полуосью. Поскольку она имеет первый порядок роста, то [20] при любом $\varepsilon>0$

$$
\sup _{0 \leqslant \alpha \leqslant \pi} \int_{0}^{\infty}\left\|G_{\varepsilon}\left(r e^{i \alpha}\right)\right\|_{1} d r<\infty .
$$

По теореме Седлецкого [21] координаты вектор-функции $G_{\varepsilon}(z)$ принадлежат классу Харди $H_{+}^{1}$ и поэтому [8]

$$
\int_{\mathbb{R}} G_{\varepsilon}(x) d x=\int_{\mathbb{R}} \frac{e^{i(a+\varepsilon) x} \mathcal{F}(x)}{x-\lambda} d x=0, \quad \lambda \in \mathbb{C}_{-}, \quad \varepsilon>0 .
$$

Это означает, что вектор-функция $e^{i(a+\varepsilon) x} \mathcal{F}(x)$, которая в силу условия 1$)$ принадлежит ${ }^{*} L_{2}\left(w^{-2}\right)$, на самом деле (теорема $\left.\mathrm{C}\right)$ принадлежит ${ }^{*} H_{+}^{2}\left(\mathbb{C}^{n}, w^{-2}\right)$, т. e.

$$
e^{i(a+\varepsilon) x} \mathcal{F}(x) w_{+}^{-1}(x+i 0) \in{ }^{*} H_{+}^{2}\left(\mathbb{C}^{n}\right)
$$

при любом $\varepsilon>0$. Отсюда вытекает первое включение (1.16). Применяя аналогичные рассуждения к аналитической в $\mathbb{C}_{-}$вектор-функции $e^{-i \varepsilon z} \mathcal{F}(z)(z-\lambda)^{-1}$, $\lambda \in \mathbb{C}_{+}$, мы докажем второе включение (1.16). Теорема доказана.

Для произвольного веса $w^{2} \in \mathcal{M}_{n}^{2}$ через $\mathcal{B}_{a}\left(w^{2}\right)$ обозначим линейное множество целых вектор-функций $\mathcal{F}(z)$ экспоненциального типа, удовлетворяющих условиям 1), 2) теоремы 1.3. Из теоремы 1.1 вытекает

СлЕДСтвиЕ 1.1. Линейное пространство $\mathcal{B}_{a}\left(w^{2}\right)$ относительно скалярного произведения

$$
(\mathcal{F}, G):=\int_{\mathbb{R}}\left(\mathcal{F}(x) w^{-2}(x), G(x)\right)_{\mathbb{C}^{n}} d x, \quad \mathcal{F}, G \in \mathcal{B}_{a}\left(w^{2}\right),
$$

является гильбертовым пространством целых вектор-функиий.

Следующее простое утверждение будет неоднократно использовано далее. Напомним, что $B_{a}^{*}$ - оператор вида (1.4) в пространстве $\mathcal{K}_{a}, g_{k}^{a}$ - векторы, определяемые равенствами (1.6) в случае $\Theta=e^{i a z} E_{n}$.

ЛЕмма 1.2. Пусть $\Theta \in J_{n}, w^{2} \in \mathcal{M}_{n}^{2}, a$ - экспоненциалъный тип матрииљ-функиии $\Theta$. Тогда имеют место равенства

$$
\mathbb{P}_{\Theta}\left(I+z B_{a}^{*}\right)^{-1} g_{k}^{a}=\left(I+z B^{*}\right)^{-1} g_{k}^{\Theta}, \quad 1 \leqslant k \leqslant n, \quad z \in \mathbb{C},
$$

где оператор $B^{*}$ задается формулой (1.4), $g_{k}^{\Theta}$ определяются равенствами (1.6).

ДокАЗАтЕльство. В силу леммы 1.1 в случае $\Theta(z)=e^{i a z} E_{n}, w^{2} \in \mathcal{M}_{n}^{2}$ имеем

$$
\left(I+z B_{a}^{*}\right)^{-1} g_{k}^{a}=\mathbb{P}_{a} \frac{w_{+}^{k}(x+i 0)}{x+z}, \quad 1 \leqslant k \leqslant n, \quad z \in \mathbb{C},
$$

где $\mathbb{P}_{a}$ - ортопроектор из $H_{+}^{2}\left(\mathbb{C}^{n}\right)$ на $\mathcal{K}_{a}$. Учитывая $(1.14)$, применим к обеим частям последнего равенства ортопроектор $\mathbb{P}_{\Theta}$. Мы получим равенства

$$
\mathbb{P}_{\Theta}\left(I+z B_{a}^{*}\right)^{-1} g_{k}^{a}=\mathbb{P}_{\Theta} \frac{w_{+}^{k}(x+i 0)}{x+z}=\left(I+z B^{*}\right)^{-1} g_{k}^{\Theta}, \quad 1 \leqslant k \leqslant n,
$$

которые и требовалось доказать. 
1.4. Для формулировки следующего результата введем обозначение

$$
\widetilde{w}(z):= \begin{cases}w_{+}(z), & z \in \mathbb{C}_{+}, \\ w_{+}(x+i 0), & x \in \mathbb{R}, \\ w_{-}(z), & z \in \mathbb{C}_{-} .\end{cases}
$$

Кроме того, положим $\mathbb{R}+i \varepsilon:=\{x+i \varepsilon: x \in \mathbb{R}\}$ для любого вещественного $\varepsilon$.

Tеорема 1.4. Пусть $\Theta \in J_{n}, w^{2} \in \mathcal{M}_{n}^{2}$, onepamop $B^{*}$ deйствует no фбормуле (1.4), векторы $g_{k}^{\Theta}$ определяются равенствами (1.6). Тогда:

1) каждой вектор-функции l на прямой $\mathbb{R}+i \varepsilon$ такой, что

$$
\int_{\mathbb{R}+i \varepsilon}\|\widetilde{w}(z) l(z)\|_{\mathbb{C}^{n}}^{2} d z<\infty, \quad l=\operatorname{col}\left(l_{k}(z)\right)_{k=1}^{n},
$$

интегральное преобразование

$$
\varphi:=\int_{\mathbb{R}+i \varepsilon} \sum_{k=1}^{n}\left(I-z B^{*}\right)^{-1} g_{k}^{\Theta} l_{k}(z) d z
$$

ставит в соответствие элемент $\varphi \in \mathcal{K}_{\Theta}$, причем справедлива оценка

$$
\|\varphi\|_{\mathcal{K}_{\Theta}}^{2} \leqslant M \int_{\mathbb{R}+i \varepsilon}\|\widetilde{w}(z) l(z)\|_{\mathbb{C}^{n}}^{2} d z
$$

2) существует подпространство L функиий $l$ такое, что сужение отображения (1.18) на L биективно отображает $L$ на $\mathcal{K}_{\Theta}$ и справедлива оценка

$$
\int_{\mathbb{R}+i \varepsilon}\|\widetilde{w}(z) l(z)\|_{\mathbb{C}^{n}}^{2} d z \leqslant M_{1}\|\varphi\|_{\mathcal{K}_{\Theta}}^{2}, \quad l \in L .
$$

ДокАЗАтельство. Шаг 1. Рассмотрим сначала случай $\varepsilon \geqslant 0$. Интегральное преобразование (1.18) первоначально определяется для финитных непрерывных вектор-функций $l$. Поскольку интеграл понимается как предел римановых сумм, то $\varphi \in \mathcal{K}_{\Theta}$. С учетом (1.18) для любого $h \in \mathcal{K}_{\Theta}$ имеем оценку

$$
\begin{aligned}
|(\varphi, h)|^{2} & =\left|\int_{\mathbb{R}+i \varepsilon} \sum_{k=1}^{n}\left(\left(I-z B^{*}\right)^{-1} g_{k}^{\Theta}, h\right) l_{k}(z) d z\right|^{2} \\
& =4 \pi^{2}\left|\int_{\mathbb{R}+i \varepsilon} \sum_{k=1}^{n}\left\{\mathcal{B}(z, h) w_{+}^{-1}(z)\right\}_{k}\left\{w_{+}(z) l_{k}(z)\right\}_{k} d z\right|^{2},
\end{aligned}
$$

где $\{\cdot\}_{k}$ обозначает $k$-ю координату столбца (строки), заключенного в фигурные скобки. Поэтому

$$
\begin{aligned}
|(\varphi, h)|^{2} & \leqslant 4 \pi^{2}\left(\int_{\mathbb{R}+i \varepsilon}\left\|\mathcal{B}(z, h) w_{+}^{-1}(z)\right\|_{\mathbb{C}^{n}}\left\|w_{+}^{-1}(z) l(z)\right\|_{\mathbb{C}^{n}} d z\right)^{2} \\
& \leqslant 4 \pi^{2} \int_{\mathbb{R}+i \varepsilon}\left\|\mathcal{B}(z, h) w_{+}^{-1}(z)\right\|_{\mathbb{C}^{n}}^{2} d z \int_{\mathbb{R}_{+i \varepsilon}}\left\|w_{+}^{-1}(z) l(z)\right\|_{\mathbb{C}^{n}}^{2} d z .
\end{aligned}
$$

Из (1.11) и теоремы С вытекает неравенство

$$
\int_{\mathbb{R}+i \varepsilon}\left\|\mathcal{B}(z, h) w_{+}^{-1}(z)\right\|_{\mathbb{C}^{n}}^{2} d z \leqslant \int_{\mathbb{R}+i \varepsilon}\left\|h^{*}(z)\right\|_{\mathbb{C}^{n}}^{2} d z+M \int_{\mathbb{R}}\left\|h^{*}(x)\right\|_{\mathbb{C}^{n}}^{2} d x .
$$


Как уже отмечалось, в силу (1.14) каждый элемент $h$ изображается интегралом Фурье-Лапласа. Из равенства Парсеваля для преобразования Фурье следует оценка

$$
\int_{\mathbb{R}+i \varepsilon}\left\|h^{*}(z)\right\|_{\mathbb{C}^{n}}^{2} d z \leqslant M_{1}(\varepsilon)\|h\|_{\mathcal{K}_{\Theta}}^{2}
$$

поэтому

$$
\int_{\mathbb{R}+i \varepsilon}\left\|\mathcal{B}(z, h) w_{+}^{-1}(z)\right\|_{\mathbb{C}^{n}}^{2} d z \leqslant M_{2}\|h\|_{\mathcal{K}_{\Theta}}^{2},
$$

и мы можем вернуться к оценке скалярного произведения:

$$
|(\varphi, h)|^{2} \leqslant M_{3}\|h\|_{\mathcal{K}_{\Theta}}^{2} \int_{\mathbb{R}+i \varepsilon}\left\|w_{+}^{-1}(z) l(z)\right\|^{2} d z .
$$

Тем самым утверждение 1) теоремы доказано.

Шаг 2. Обозначим через $L$ множество вектор-функций вида

$$
l_{0}(z)=-\frac{1}{2 \pi i} w_{+}^{-1}(z) \varphi_{0}(z-2 i \varepsilon)
$$

где $\varphi_{0}-$ произвольная функция из модельного пространства $\mathcal{K}_{\Theta}$. Нетрудно видеть, что $l_{0}$ удовлетворяет условию (1.17):

$$
\int_{\mathbb{R}+i \varepsilon}\left\|w_{+}(z) l_{0}(z)\right\|_{\mathbb{C}^{n}}^{2} d z=\frac{1}{4 \pi^{2}} \int_{\mathbb{R}}\left\|\varphi_{0}(x-i \varepsilon)\right\|_{\mathbb{C}^{n}}^{2} d x<\infty .
$$

Вычислим теперь значение $\varphi$ интегрального преобразования (1.18) для указанной функции $l_{0}$. Для каждого $h \in \mathcal{K}_{\Theta}$ имеем

$$
\begin{aligned}
(\varphi, h)_{\mathcal{K}_{\Theta}} & =\int_{\mathbb{R}+i \varepsilon} \sum_{k=1}^{n}\left(\left(I-z B^{*}\right)^{-1} g_{k}^{\Theta}, h\right) l_{k}^{0}(z) d z \\
& =-\frac{1}{2 \pi i} \int_{\mathbb{R}+i \varepsilon} \sum_{k=1}^{n}\left(\left(I-z B^{*}\right)^{-1} g_{k}^{\Theta}, h\right) \sum_{j=1}^{n}{ }^{k j} w_{+}^{-1}(z) \varphi_{0}^{j}(z-2 i \varepsilon) d z \\
& =\int_{\mathbb{R}+i \varepsilon} \sum_{j=1}^{n}\left\{\mathcal{B}(z, h) w_{+}^{-1}(z)\right\}_{j} \varphi_{0}^{j}(z-2 i \varepsilon) d z,
\end{aligned}
$$

где $l_{0}=\operatorname{col}\left(l_{k}^{0}\right)_{k=1}^{n}, \varphi_{0}=\operatorname{col}\left(\varphi_{0}^{k}\right)_{k=1}^{n},{ }^{k j} w_{+}^{-1}(z)$ - элемент матрицы $w_{+}^{-1}(z)$. С учетом (1.11) полученную формулу можно переписать в виде

$$
(\varphi, h)_{\mathcal{K}_{\Theta}}=\int_{\mathbb{R}} \sum_{j=1}^{n}\left(h_{j}^{*}(x+i \varepsilon)+\psi_{j}(x+i \varepsilon)\right) \varphi_{0}^{j}(x-i \varepsilon) d x,
$$

где $h=\operatorname{col}\left(h_{j}\right)_{j=1}^{n}$, а функции $\psi_{j}$ принадлежат $H_{+}^{2}$. Поэтому

$$
\begin{aligned}
(\varphi, h)_{\mathcal{K}_{\Theta}} & =\int_{\mathbb{R}} \sum_{j=1}^{n} h_{j}^{*}(x+i \varepsilon) \varphi_{0}^{j}(x-i \varepsilon) d x=\int_{\mathbb{R}}\left(\varphi_{0}(x-i \varepsilon), h(x+i \varepsilon)\right)_{\mathbb{C}^{n}} d x \\
& =\int_{\mathbb{R}}\left(\varphi_{0}(x), h(x)\right)_{\mathbb{C}^{n}} d x=\left(\varphi_{0}, h\right)_{\mathcal{K}_{\Theta}} .
\end{aligned}
$$


Заметим, что предпоследнее равенство в этой выкладке снова вытекает из представления $\varphi_{0}, h$ в виде интегралов Фурье-Лапласа и унитарности преобразования Фурье. Таким образом, $\varphi=\varphi_{0}$, и оценка (1.19) легко вытекает из (1.20).

Шаг 3. Рассмотрим теперь случай прямой $\mathbb{R}-i \varepsilon, \varepsilon>0$ (т. е. $\mathbb{R}+i \varepsilon$ заменяется прямой $\mathbb{R}-i \varepsilon, \varepsilon>0$, в формулировке теоремы 1.4). Доказательство утверждения 1) теоремы аналогично уже изложенному. Мы снова получаем оценку

$$
|(\varphi, h)|^{2} \leqslant \pi^{2} \int_{\mathbb{R}-i \varepsilon}\left\|\mathcal{B}(z, h) w_{-}^{-1}(z)\right\|_{\mathbb{C}^{n}}^{2} d z \int_{\mathbb{R}-i \varepsilon}\left\|w_{-}^{-1}(z) l(z)\right\|_{\mathbb{C}^{n}}^{2} d z,
$$

и осталось заметить, что из (1.9) и теоремы С выводится неравенство

$$
\int_{\mathbb{R}-i \varepsilon}\left\|\mathcal{B}(z, h) w_{-}^{-1}(z)\right\|_{\mathbb{C}^{n}}^{2} d z \leqslant M\|h\|_{\mathcal{K}_{\Theta}}^{2}
$$

Утверждение 2) теоремы докажем сначала в частном случае $\Theta(z)=e^{i a z} E_{n}$, $w^{2} \in \mathcal{M}_{n}^{2}$. Из (1.9) следует, что при любом $z \in \mathbb{C}_{-}$и любом $h \in \mathcal{K}_{a}$

$$
\mathcal{B}(z, h) w_{-}^{-1}(z)=-\frac{1}{2 \pi i} \int_{\mathbb{R}} \frac{h^{*}(x) w_{+}(x+i 0)}{x-z} d x w_{-}^{-1}(z) .
$$

Из теоремы Винера-Пэли вытекает представление

$$
\mathcal{B}(z, h) w_{-}^{-1}(z)=\frac{1}{\sqrt{2 \pi}} \int_{0}^{\infty} e^{-i z t} G(h, t) d t, \quad z \in \mathbb{C}_{-}, \quad h \in \mathcal{K}_{a},
$$

где вектор-функция $G(h, t)$ принадлежит ${ }^{*} L_{2}^{n}\left(\mathbb{R}_{+}\right)$. Рассмотрим теперь линейный оператор, который каждому $h \in \mathcal{K}_{a}$ ставит в соответствие сужение $\overline{G(h, t)}$ на $[0, a]$, т. е.

$$
(T h)(t)=\overline{G(h, t)}, \quad 0 \leqslant t \leqslant a, \quad h \in \mathcal{K}_{a} .
$$

Из (1.21) вытекает ограниченность оператора $T$ из $\mathcal{K}_{a}$ в пространство ${ }^{*} L_{2}^{n}(0, a)$. Докажем теперь, что оператор $T$ ограничен снизу. Запишем равенство правых частей (1.22) и (1.23). После перехода к пределу при $z \rightarrow y$ и умножения на $w_{-}(y-i 0)$ получим

$$
\mathcal{P}_{-}\left(h^{*}(x) w_{+}(x+i 0)\right)=\int_{0}^{\infty} e^{-i y t} G(h, t) d t w_{-}(y-i 0) .
$$

Это равенство можно переписать в виде

$$
\begin{aligned}
& \left(h^{*}(y)-\left(\mathcal{P}_{+} h^{*}(x) w_{+}(x+i 0)\right) w_{+}^{-1}(y+i 0)\right) w_{+}(y+i 0) \\
& \quad-\int_{0}^{a} e^{-i y t} G(h, t) d t w_{-}(y-i 0)=\int_{a}^{\infty} e^{-i y t} G(h, t) d t w_{-}(y-i 0) .
\end{aligned}
$$

Умножим обе части этого равенства на $e^{i y a}$, после чего первое слагаемое слева будет принадлежать пространству ${ }^{*} H_{+}^{2}\left(\mathbb{C}^{n}, w^{-2}\right)$, а выражение справа - пространству ${ }^{*} H_{-}^{2}\left(\mathbb{C}^{n}, w^{-2}\right)$. Поэтому если предположить, что $T h_{n} \rightarrow 0$ для некоторой последовательности $h_{n} \in \mathcal{K}_{a}$, то

$$
\int_{0}^{a} e^{-i y t} G\left(h_{n}, t\right) d t w_{-}(y-i 0) e^{i a y} \rightarrow 0, \quad n \rightarrow \infty
$$


в метрике пространства ${ }^{*} L_{2}^{n}\left(w^{-2}\right)$ и, стало быть,

$$
e^{i y a}\left(h_{n}^{*}(y)-\left(\mathcal{P}_{+} h_{n}^{*}(x) w_{+}(x+i 0)\right) w_{+}^{-1}(y+i 0)\right) \rightarrow 0, \quad n \rightarrow \infty,
$$

по норме пространства строк ${ }^{*} H_{+}^{2}\left(\mathbb{C}^{n}\right)$. Теперь из $(1.11)$ следует, что $h_{n} \rightarrow 0$, $n \rightarrow \infty$, т. е. оператор $T$ ограничен снизу.

Шаг 4. Докажем теперь, что образ оператора $T$ совпадает со всем пространством ${ }^{*} L_{2}^{n}(0, a)$. Предположим, что существует функция $f \in{ }^{*} L_{2}^{n}(0, a)$ такая, что для всех $h \in \mathcal{K}_{a}$

$$
\begin{gathered}
0=(G(h, t), f(t))=\int_{0}^{a} \sum_{k=1}^{n} G_{k}(h, t) \overline{f_{k}(t)} d t=\sum_{k=1}^{n} \int_{\mathbb{R}}\left(\mathcal{F} G_{k}\right)(t) \overline{\left(\mathcal{F} f_{k}\right)(t)} d t, \\
G(h, t)=\operatorname{row}\left(G_{k}(h, t)\right)_{k=1}^{n}, \quad f=\operatorname{row}\left(f_{k}\right)_{k=1}^{n},
\end{gathered}
$$

где через $\mathcal{F}$ обозначено преобразование Фурье с ядром $\exp \{-i x t\}$. Учитывая формулы (1.23) и (1.8), получим

$$
\begin{aligned}
0 & =\sum_{k=1}^{n} \int_{\mathbb{R}}\left\{\mathcal{B}_{a}(x, h) w_{-}^{-1}(x-i 0)\right\}_{k} \int_{0}^{a} e^{i x t} \overline{f_{k}(t)} d t d x \\
& =-\frac{1}{2 \pi i} \sum_{k=1}^{n} \int_{\mathbb{R}} \sum_{j=1}^{n}\left(\left(I-x B_{a}^{*}\right)^{-1} g_{j}^{a}, h\right)^{j k} w_{-}^{-1}(x-i 0) \int_{0}^{a} e^{i x t} \overline{f_{k}(t)} d t d x \\
& =-\frac{1}{2 \pi i}\left(\int_{\mathbb{R}} \sum_{j=1}^{n}\left(I-x B_{a}^{*}\right)^{-1} g_{j}^{a} l_{j}(x) d x, h\right), \quad h \in \mathcal{K}_{a} .
\end{aligned}
$$

Отметим, что в последней формуле мы ввели обозначение

$$
l(x)=\operatorname{col}\left(l_{j}(x)\right)_{j=1}^{n}, \quad l_{j}(x)=\sum_{k=1}^{n}{ }^{j k} w_{-}^{-1}(x-i 0) \int_{0}^{a} e^{i x t} \overline{f_{k}(t)} d t .
$$

Заметим, что

$$
\int_{\mathbb{R}}\left(w^{2}(x) l(x), l(x)\right)_{\mathbb{C}^{n}} d x=\int_{\mathbb{R}} \sum_{k=1}^{n}\left|\int_{0}^{a} e^{i x t} \overline{f_{k}(t)} d t\right|^{2} d x=2 \pi \sum_{k=1}^{n} \int_{0}^{a}\left|f_{k}(t)\right|^{2} d t<\infty .
$$

Поэтому из теоремы С вытекает разложение

$$
l=l_{+}+l_{-}, \quad l_{ \pm} \in H_{ \pm}^{2}\left(\mathbb{C}^{n}, w^{2}\right) .
$$

Для любого $h \in \mathcal{K}_{a}$ имеем

$$
\begin{aligned}
\left(\int_{\mathbb{R}} \sum_{j=1}^{n}\left(I-x B_{a}^{*}\right)^{-1} g_{j}^{a} l_{j}^{-}(x) d x, h\right) & \\
= & \sum_{k=1}^{n} \int_{\mathbb{R}}\left\{\mathcal{B}_{a}(h, x) w_{-}^{-1}(x-i 0)\right\}_{k}\left\{w_{-}(x-i 0) l_{-}(x)\right\}_{k} d x=0
\end{aligned}
$$

поскольку оба множителя под интегралом принадлежат пространству $H_{-}^{2}$. Поэтому из последнего равенства, (1.24) и (1.26) следует, что

$$
\int_{\mathbb{R}} \sum_{k=1}^{n}\left(I-x B_{a}^{*}\right)^{-1} g_{k}^{a} l_{k}^{+}(x) d x=0, \quad l_{+}=\operatorname{col}\left(l_{k}^{+}\right)_{k=1}^{n},
$$


где $l_{+}=\mathcal{P}_{+} l$, a $l$ представима в виде (1.25). Подействуем на обе части последнего равенства оператором $B_{a}^{*}\left(I-\mu B_{a}^{*}\right)^{-1}$ :

$$
\begin{aligned}
0 & =\int_{\mathbb{R}} \sum_{k=1}^{n} B_{a}^{*}\left(I-\mu B_{a}^{*}\right)^{-1}\left(I-x B_{a}^{*}\right)^{-1} g_{k}^{a} l_{k}^{+}(x) d x \\
& =\int_{\mathbb{R}} \sum_{k=1}^{n} \frac{\left(I-\mu B_{a}^{*}\right)^{-1} g_{k}^{a}-\left(I-x B_{a}^{*}\right)^{-1} g_{k}^{a}}{-x+\mu} l_{k}^{+}(x) d x .
\end{aligned}
$$

Если предположить, что $\mu \in \mathbb{C}_{-}$, отсюда выводим равенство

$$
\int_{\mathbb{R}} \sum_{k=1}^{n} \frac{\left(I-x B_{a}^{*}\right)^{-1} g_{k}^{a}}{x-\mu} l_{k}^{+}(x) d x=0 .
$$

Таким образом, для любого $h \in \mathcal{K}_{a}$ и любого $\mu \in \mathbb{C}_{-}$

$$
\begin{aligned}
0 & =-\frac{1}{2 \pi i} \int_{\mathbb{R}}(x-\mu)^{-1} \sum_{k=1}^{n}\left(\left(I-x B_{a}^{*}\right)^{-1} g_{k}^{a}, h\right) l_{+}^{k}(x) d x \\
& =\int_{\mathbb{R}}(x-\mu)^{-1} \sum_{j=1}^{n}\left\{\mathcal{B}_{a}(x, h) w_{+}^{-1}(x+i 0)\right\}_{j}\left\{w_{+}(x+i 0) l_{+}(x)\right\}_{j} d x
\end{aligned}
$$

где, как и раньше, $\{\cdot\}_{j}$ обозначает $j$-ю координату вектора, заключенного в скобки. Если теперь учесть формулу (1.11), то из последнего равенства получим

$$
\int_{\mathbb{R}}(x-\mu)^{-1} \sum_{j=1}^{n} h_{j}^{*}(x)\left\{w_{+}(x+i 0) l_{+}(x)\right\}_{j} d x=0
$$

для любого $\mu \in \mathbb{C}_{-}$и любого $h \in \mathcal{K}_{a}$. Отсюда вытекает, что скалярное произведение $\left(w_{+}(x+i 0) l_{+}(x), h\right)_{\mathbb{C}^{n}}$ принадлежит классу Харди $H_{+}^{1}$ и, стало быть,

$$
\int_{\mathbb{R}}\left(w_{+}(x+i 0) l_{+}(x), h(x)\right)_{\mathbb{C}^{n}} d x=0, \quad h \in \mathcal{K}_{a} .
$$

Поэтому из определения модельного пространства $\mathcal{K}_{a}$ следует справедливость представления

$$
w_{+}(x+i 0) l_{+}(x)=\int_{a}^{\infty} e^{i x t} \psi(t) d t, \quad \psi=\operatorname{col}\left(\psi_{k}\right)_{k=1}^{n}, \quad \psi_{k} \in L_{2}(a, \infty) .
$$

Учитывая (1.25), равенство (1.26) перепишем в виде

$w_{-}^{-1}(x+i 0) \int_{0}^{a} e^{i x t} \overline{f(t)} d t=w_{+}^{-1}(x+i 0) \int_{a}^{\infty} e^{i x t} \psi(t) d t+w_{-}^{-1}(x-i 0) F_{-}(x-i 0)$,

где $F_{-}:=w_{-}(x-i 0) l_{-}(x) \in H_{-}^{2}\left(\mathbb{C}^{n}\right)$. Умножая обе части равенства (1.27) на $e^{-i x a}$, приходим к выводу, что

$$
w_{+}^{-1}(x+i 0) \int_{a}^{\infty} e^{i x t} \psi(t) d t=0 .
$$

Теперь из (1.27) следует, что $f=0$, т. е. оператор $T$ отображает $\mathcal{K}_{a}$ на $L_{2}^{n}(0, a)$ биективно. 
Шаг 5. Рассмотрим снова преобразование (1.18) в случае $\Theta(z)=e^{i a z} E_{n}$, т. е.

$$
\varphi=\int_{\mathbb{R}-i \varepsilon} \sum_{k=1}^{n}\left(I-z B_{a}^{*}\right)^{-1} g_{k}^{a} l_{k}(z) d z .
$$

Предположим, что $l(z)$ - произвольная функция вида (1.25). Таким образом, имеем представление

$$
l(z)=\frac{1}{\sqrt{2 \pi}} w_{-}^{-1}(z) \int_{0}^{a} e^{i z t} f(t) d t, \quad f \in L_{2}^{n}(0, a) .
$$

Учитывая (1.23) и определение оператора $T$, для любого $h \in \mathcal{K}_{a}$ получим

$$
\begin{aligned}
(\varphi, h)_{\mathcal{K}_{a}} & =\int_{\mathbb{R}-i \varepsilon} \sum_{j=1}^{n}\left\{\mathcal{B}(z, h) w_{-}^{-1}(z)\right\}_{j} \frac{1}{\sqrt{2 \pi}} \int_{0}^{a} e^{i z t} f_{j}(t) d t d z \\
& =\int_{\mathbb{R}} \sum_{j=1}^{n}\left(\mathcal{F} e^{-\varepsilon t} G_{j}(h, t)\right)(x) \overline{\left(\mathcal{F} e^{\varepsilon t} \overline{f_{j}(t)}\right)(x)} d x \\
& =\int_{0}^{a} \sum_{j=1}^{n} G_{j}(h, t) f_{j}(t) d t=(f, T h)=\left(T^{*} f, h\right)_{\mathcal{K}_{a}} .
\end{aligned}
$$

Таким образом, $\varphi=T^{*} f$, и, стало быть, имеет место оценка (1.19):

$$
\begin{aligned}
\int_{\mathbb{R}-i \varepsilon}\left\|w_{-}(z) l(z)\right\|_{\mathbb{C}^{n}}^{2} d z & =\int_{\mathbb{R}}\left\|\frac{1}{\sqrt{2 \pi}} \int_{0}^{a} e^{i x t} e^{\varepsilon t} f(t) d t\right\|_{\mathbb{C}^{n}}^{2} d x \\
& \leqslant C_{1}\|f\|^{2} \leqslant C_{2}\left\|T^{*} f\right\|_{\mathcal{K}_{a}}^{2}=C_{2}\|\varphi\|_{\mathcal{K}_{a}}^{2} .
\end{aligned}
$$

Итак утверждение 2) теоремы доказано полностью в частном случае $\Theta(z)=$ $e^{i a z} E_{n}$.

Пусть теперь $\Theta \in J_{n}$ и $a-$ экспоненциальный тип $\Theta$. Отображение (1.28) биективно отображает пространство $L_{a}$ функций $l$ вида $(1.25)$ на $\mathcal{K}_{a}$. Поскольку $\mathcal{K}_{\Theta} \subseteq \mathcal{K}_{a}$ (см. (1.14)), то (1.28) биективно переводит $\mathcal{K}_{\Theta}$ в некоторое подпространство $L_{\Theta} \subseteq L_{a}$ функций $l$, удовлетворяющих условию (1.17). Другими словами, каждая функция $\varphi \in \mathcal{K}_{\Theta}$ представима в виде $(1.28)$ с некоторой функцией $l \in L_{\Theta}$. Подействуем проектором $\mathbb{P}_{\Theta}$ на обе части $(1.28)$ с такими $\varphi$ и $l$. Из леммы 1.2 следует, что

$$
\varphi=\mathbb{P}_{\Theta} \varphi=\int_{\mathbb{R}-i \varepsilon} \sum_{k=1}^{n}\left(I-z B^{*}\right)^{-1} g_{k}^{\Theta} l_{k}(z) d z, \quad l=\operatorname{col}\left(l_{k}\right)_{k=1}^{n} \in L_{\Theta} .
$$

Заметим, что неравенство (1.19) в этом случае снова следует из (1.29). Теорема доказана.

1.5. В этом пункте мы сведем общую задачу об описании генераторов $C_{0}$-полугрупп в множестве $w$-возмущений операторов класса $\Sigma^{(\exp )}$ к исследованию специальных конечномерных возмущений модельных операторов $B^{*}$ в пространстве $\mathcal{K}_{\Theta}$.

Итак, пусть неограниченный плотно заданный оператор $A$ есть $w$-возмущение оператора $A_{0} \in \Sigma^{(\exp )}$ (см. введение) ранга $n$, т. е.

$$
A^{-1} h=A_{0}^{-1} h+\sum_{k=1}^{n}\left(h, f_{k}\right) g_{k}, \quad h \in \mathfrak{H},
$$


и существует вес $w^{2} \in \mathcal{M}_{n}^{2}$ такой, что $\left\|\mathcal{A}_{0}(x, h) w^{-1}(x)\right\|_{\mathbb{C}^{n}}$ принадлежит $L_{2}(\mathbb{R})$ и справедлива оценка снизу

$$
\begin{gathered}
m\|h\|^{2} \leqslant \int_{\mathbb{R}}\left\|\mathcal{A}_{0}(x, h) w^{-1}(x)\right\|_{\mathbb{C}^{n}}^{2} d x, \\
\mathcal{A}_{0}(x, h):=\operatorname{row}\left\{\left(A_{0}\left(A_{0}-z I\right)^{-1} g_{k}, h\right)\right\}_{k=1}^{n}
\end{gathered}
$$

с некоторой константой $m>0$. Обозначим через $a$ экспоненциальный тип целой оператор-функции $\left(A_{0}-z I\right)^{-1}$. Поскольку $A_{0}$ генерирует $C_{0}$-полугруппу $V_{+}(t):=\exp \left\{i A_{0} t\right\}$, имеет место представление

$$
i\left(A_{0}-z I\right)^{-1}=\int_{0}^{a} e^{-i z t} V_{+}(t) d t .
$$

Если обозначить $A_{0}(z, h)=\operatorname{row}\left(a_{k}(z)\right)_{k=1}^{n}$, то отсюда следует, что $h\left(a_{k},-\frac{\pi}{2}\right) \leqslant 0$, $h\left(a_{k}, \frac{\pi}{2}\right) \leqslant a, k=1,2, \ldots, n$. Таким образом, в силу теоремы 1.3 каждая строка $A_{0}(z, h)$ находится в образе преобразования $\mathcal{B}_{a}$, т. е.

$$
-\frac{1}{2 \pi i} \mathcal{A}_{0}(z, h)=\mathcal{B}_{a}(z, S h), \quad h \in \mathfrak{H},
$$

где $S$ - линейный оператор из $\mathfrak{H}$ в $\mathcal{K}_{a}$. Из леммы Гельфанда о выпуклых функционалах [22], [23] вытекает существование константы $M>0$ такой, что

$$
\int_{\mathbb{R}}\left\|\mathcal{A}_{0}(x, h) w^{-1}(x)\right\|_{\mathbb{C}^{n}}^{2} d x \leqslant M\|h\|^{2}, \quad h \in \mathfrak{H} .
$$

Поэтому из (1.31) и теоремы 1.1 для $\mathcal{B}_{a}(z, h)$ следует, что для любого вектора $h \in \mathfrak{H}$ имеем

$$
\|h\|_{\mathfrak{H}}^{2} \asymp \int_{\mathbb{R}}\left\|\mathcal{A}_{0}(x, h) w^{-1}(x)\right\|_{\mathbb{C}^{n}}^{2} d x=4 \pi^{2} \int_{\mathbb{R}}\left\|\mathcal{B}_{a}(z, S h) w^{-1}(x)\right\|_{\mathbb{C}^{n}}^{2} d x \asymp\|S h\|_{\mathcal{K}_{a}}^{2} .
$$

Эти двусторонние оценки означают, что оператор $S$ есть изоморфизм пространства $\mathfrak{H}$ на свой образ $\mathcal{L} \subseteq \mathcal{K}_{a}$. Перепишем теперь (1.31) в виде

$$
\left(\left(I-z A_{0}^{-1}\right)^{-1} g_{k}, h\right)_{\mathfrak{H}}=\left(\left(I-z B_{a}^{*}\right)^{-1} g_{k}^{a}, S h\right)_{\mathcal{K}_{a}}, \quad 1 \leqslant k \leqslant n, \quad h \in \mathfrak{H}, \quad z \in \mathbb{C} .
$$

Отсюда выводим следующую цепочку равенств:

$$
\begin{aligned}
\left(\left(I-z A_{0}^{-1}\right)^{-1} g_{k},\left(A_{0}^{-1}\right)^{*} h\right)_{\mathfrak{H}}=z^{-1}\left(\left(I-z A_{0}^{-1}\right)^{-1} g_{k}-g_{k}, h\right)_{\mathfrak{H}} \\
\quad=z^{-1}\left(\left(I-z B_{a}^{*}\right)^{-1} g_{k}^{a}-g_{k}^{a}, S h\right)_{\mathcal{K}_{a}}=\left(\left(I-z B_{a}^{*}\right)^{-1} g_{k}^{a}, B_{a} S h\right)_{\mathcal{K}_{a}},
\end{aligned}
$$

где $1 \leqslant k \leqslant n, z \in \mathbb{C}$. Поэтому образ $\mathcal{L}$ оператора $S$ инвариантен относительно $B_{a}$ и $S\left(A_{0}^{-1}\right)^{*}=\left(B_{a} \mid \mathcal{L}\right) S$. Известно [17], что существуют матрицы-функции $\Theta, \widetilde{\Theta} \in J_{n}$ такие, что:

1) $\Theta(z) \widetilde{\Theta}(z)=e^{i a z} E_{n}$

2) $\mathcal{L}=\mathcal{K}_{\Theta}$

3) $B_{a} \mid \mathcal{L}$ задается формулой (1.3).

Используя лемму 1.2, равенство (1.32) перепишем в виде

$$
\left(\left(I-z A_{0}^{-1}\right)^{-1} g_{k}, h\right)_{\mathfrak{H}}=\left(\left(I-z B^{*}\right)^{-1} g_{k}^{\Theta}, S h\right)_{\mathcal{K}_{\Theta}}, \quad 1 \leqslant k \leqslant n,
$$

где векторы $g_{k}^{\Theta}, 1 \leqslant k \leqslant n$, определяются равенствами (1.6). Таким образом, оператор $Q:=\left(S^{*}\right)^{-1}$ является изоморфизмом пространства $\mathfrak{H}$ на модельное 
пространство $\mathcal{K}_{\Theta}$, причем $B^{*}=Q A_{0}^{-1} Q^{-1}$, где $B^{*}$ действует по формуле (1.4) и, кроме того, $Q g_{k}=g_{k}^{\Theta}, 1 \leqslant k \leqslant n$. Поэтому если $A-w$-возмущение оператора $A_{0} \in \Sigma^{(\exp )}$, то

$$
Q A^{-1} h=Q A_{0}^{-1} h+\sum_{k=1}^{n}\left(h, f_{k}\right) Q g_{k}=K Q h, \quad h \in \mathfrak{H},
$$

где оператор $K$ определяется равенством

$$
K f=B^{*} f+\sum_{k=1}^{n}\left(f, f_{k}^{\Theta}\right) g_{k}^{\Theta}, \quad f \in \mathcal{K}_{\Theta}, \quad f_{k}^{\Theta}:=\left(Q^{*}\right)^{-1} f_{k}
$$

Таким образом, каждое $w$-возмущение оператора $A_{0} \in \Sigma^{(\exp )}$ порождает пару $\Theta \in J_{n}, w^{2} \in \mathcal{M}_{n}^{2}$, причем вес $w^{2}$ входит в определение $w$-возмущения, а матрица-функция $\Theta$, как это будет доказано далее, однозначно определяется из условий:

а) $\Theta$ - левый делитель матрицы-функции $e^{i a z} E_{n}$, где $a-$ экспоненциальный тип оператор-функции $\left(A_{0}-z I\right)^{-1}$;

b) имеет место включение ${ }^{2} \mathcal{A}_{0}(x, h) w_{+}^{-1}(x+i 0) \Theta(x) \in{ }^{*} H_{+}^{2}\left(\mathbb{C}^{n}\right)$ для любого $h \in \mathfrak{H}$;

c) если $\Theta_{1}-$ левый делитель $e^{i a z} E_{n}$, для которого также выполняется условие b), то $\Theta_{1}=\Theta V$, где $V \in J_{n}$.

Сформулируем теперь основной результат настоящего пункта.

ТеОрема 1.5. Пусть в гильбертовом пространстве $\mathfrak{H}$ неограниченный плотно заданный оператор А определяется формулой (1.30), причем А есть wвозмущение оператора $A_{0} \in \Sigma^{(\exp )}$. Тогда оператору $A$ отвечают матричный вес $w^{2} \in \mathcal{M}_{n}^{2}$ и матрииа-функиия $\Theta \in J_{n}$, определяемая условиями а)-с), такие, что:

1) существует изоморфизм $Q$ пространства $\mathfrak{H}$ на $\mathcal{K}_{\Theta}$ такой, что выполняются равенства

$$
B^{*}=Q A_{0}^{-1} Q^{-1}, \quad Q g_{k}=g_{k}^{\Theta}, \quad 1 \leqslant k \leqslant n,
$$

где $B^{*}$ задается формулой (1.4), $g_{k}^{\Theta}$ - векторы, определяемые равенствами (1.16) по указанным $\Theta, w^{2}$;

2) $Q A^{-1} Q^{-1}=K$, где оператор $K$ определяется формулой

$$
K f=B^{*} f+\sum_{k=1}^{n}\left(f, f_{k}^{\Theta}\right) g_{k}^{\Theta}, \quad f_{k}^{\Theta}:=\left(Q^{*}\right)^{-1} f_{k}, \quad f \in \mathcal{K}_{\Theta} .
$$

Обратно, каждая пара $\Theta \in J_{n}, w^{2} \in \mathcal{M}_{n}^{2}$ по формуле (1.34) задает оператор $K$, который в случае $\operatorname{Ker} K=\operatorname{Ker} K^{*}=\{0\}$ порождает $w$-возмущение $A:=K^{-1}$ onepamopa $A_{0}:=\left(B^{*}\right)^{-1}$ класcа $\Sigma^{(\exp )}$.

ДокАЗАтЕльство. В процессе доказательства первой части теоремы установлено, что для матрицы-функции $\Theta$ выполняются равенства (1.33). Поэтому в силу теоремы 1.2 выполняется условие b), а также

$$
\mathcal{A}_{0}(x, h) w_{-}^{-1}(x-i 0) \in{ }^{*} H_{-}^{2}\left(\mathbb{C}^{n}\right), \quad h \in \mathfrak{H} .
$$

\footnotetext{
${ }^{2}$ Матрица-функция $w_{+}(x+i 0)$ входит в факторизацию $(1.1)$ веса $w^{2}$.
} 
Пусть теперь $\mathcal{A}_{0}(x, h) w_{+}^{-1}(x+i 0) \Theta_{1}(x) \in{ }^{*} H_{+}^{2}\left(\mathbb{C}^{n}\right)$ для всех $h \in \mathfrak{H}$, где $\Theta_{1}-$ некоторый левый делитель $e^{i a z} E_{n}$. Из теоремы 1.2 и леммы 1.2 выводим, что для каждого $h \in \mathfrak{H}$ существует $f_{1} \in \mathcal{K}_{\Theta_{1}}$ такой, что

$$
\begin{aligned}
\left(\left(I-z A_{0}^{-1}\right)^{-1} g_{k}, h\right) & =\left(\left(I-z B^{*}\right)^{-1} g_{k}^{\Theta_{1}}, f_{1}\right)_{\mathcal{K}_{\Theta_{1}}} \\
& =\left(\left(I-z B_{a}^{*}\right)^{-1} g_{k}^{a}, f_{1}\right)_{\mathcal{K}_{a}}, \quad 1 \leqslant k \leqslant n .
\end{aligned}
$$

Сравнивая эти равенства с (1.32) и учитывая, что образ оператора $S$ совпадает с $\mathcal{K}_{\Theta}$, приходим к выводу, что для любого $f \in \mathcal{K}_{\Theta}$ найдется $f_{1} \in \mathcal{K}_{\Theta_{1}}$ такой, что

$$
\left(\left(I-z B_{a}^{*}\right)^{-1} g_{k}^{a}, f\right)_{\mathcal{K}_{a}}=\left(\left(I-z B^{*}\right)^{-1} g_{k}^{a}, f_{1}\right)_{\mathcal{K}_{a}}, \quad 1 \leqslant k \leqslant n .
$$

Из теоремы 1.1 следует равенство $f=f_{1}$ и, стало быть, включение $\mathcal{K}_{\Theta} \subseteq \mathcal{K}_{\Theta_{1}}$. Отсюда легко вытекает [17], что $\Theta$ - левый делитель $\Theta_{1}$, т. е. $\Theta_{1}=\Theta V, V \in J_{n}$. Таким образом, матрица-функция $\Theta \in J_{n}$ удовлетворяет условиям а)-c), и ясно, что этими условиями она определяется однозначно.

Вторая часть теоремы вытекает из теоремы 1.1.

\section{§ 2. Описание генераторов}

$$
\text { сильно непрерывных полугрупп } U_{-}(t):=\exp \{-i A t\}, t \geqslant 0
$$

2.1. Настоящий параграф посвящен описанию генераторов $C_{0}$-полугрупп $U_{-}(t):=\exp \{-i A t\}, t \geqslant 0$, где $A-w$-возмущение некоторого оператора $A_{0}$ класса $\Sigma^{(\exp )}$. В п. 1.5 было показано, что задача сводится к исследованию операторов вида

$$
K h=B^{*} h+\sum_{k=1}^{n}\left(h, f_{k}^{\Theta}\right) g_{k}^{\Theta}, \quad h \in \mathcal{K}_{\Theta}
$$

которые строятся по $\Theta \in J_{n}$ и $w^{2} \in \mathcal{M}_{n}^{2}$. Напомним, что оператор $B^{*}$ действует в $\mathcal{K}_{\Theta}$ по формуле (1.4), векторы $g_{k}^{\Theta}$ определяются равенствами $(1.6), f_{k}^{\Theta}-$ некоторые векторы из $\mathcal{K}_{\Theta}, 1 \leqslant k \leqslant n$.

В результате простых вычислений, которые мы не приводим, получаем формулу для фредгольмовой резольвенты оператора $K$ :

$$
K(I-z K)^{-1} h=B^{*}\left(I-z B^{*}\right)^{-1} h+\sum_{k=1}^{n} f_{k}(h, z)\left(I-z B^{*}\right)^{-1} g_{k}^{\Theta},
$$

где функционалы $f_{k}(h, z)$ определяются формулами

$$
\begin{gathered}
f_{k}(h, z)=\sum_{j=1}^{n} \Psi_{k j}(z)\left(\left(I-z B^{*}\right)^{-1} h, f_{j}^{\Theta}\right), \\
\Psi(z):=\Phi^{-1}(z), \quad \Phi_{k j}(z):=\delta_{k j}-z\left(\left(I-z B^{*}\right)^{-1} g_{j}^{\Theta}, f_{k}^{\Theta}\right), \quad 1 \leqslant k, j \leqslant n .
\end{gathered}
$$

Отсюда вытекает, что фредгольмов спектр вполне непрерывного оператора $K$ совпадает с последовательностью $\Lambda$ корней целой функции $\Delta(z):=\operatorname{det} \Phi(z)$. Каждому $\lambda_{k} \in \Lambda$ отвечает собственное подпространство, которое состоит из векторов вида

$$
h=\sum_{j=1}^{n} C_{j}\left(I-\lambda_{k} B^{*}\right)^{-1} g_{j}^{\Theta}, \quad \Phi\left(\lambda_{k}\right) C=0, \quad C=\operatorname{col}\left(C_{j}\right)_{j=1}^{n} .
$$


Введем обозначение

$$
F(h, z)=\operatorname{col}\left(f_{k}(h, z)\right)_{k=1}^{n}
$$

и напомним, что матрицы $w_{+}, w_{-}$взяты из факторизации $(1.1)$ веса $w^{2} \in \mathcal{M}_{n}^{2}$. Следующая теорема играет важную роль в наших построениях.

Теорема 2.1. Следующие условия эквивалентны:

1) для всех $h \in \mathcal{K}_{\Theta}$ имеет место оценка

$$
\int_{\mathbb{R}+i \varepsilon}\left\|w_{+}(z) F(h, z)\right\|_{\mathbb{C}^{n}}^{2} d z \leqslant M\|h\|^{2},
$$

где $\varepsilon \geqslant 0, M-$ некоторая константа;

2) матричныц вес $W_{\varepsilon}^{2}(x):=\Phi(x+i \varepsilon)\left(w_{+}^{*}(x+i \varepsilon) w_{+}(x+i \varepsilon)\right)^{-1} \Phi^{*}(x+i \varepsilon)$ удовлетворяет $\left(A_{2}\right)$-условию на $\mathbb{R}$.

Сформулируем также аналог этого утверждения для нижней полуплоскости.

Теорема 2.1'. Следующие условия эквивалентны:

1) для всех $h \in \mathcal{K}_{\Theta}$ имеет место оценка

$$
\int_{\mathbb{R}-i \varepsilon}\left\|w_{-}(z) F(h, z)\right\|_{\mathbb{C}^{n}}^{2} d z \leqslant M\|h\|^{2},
$$

где $\varepsilon>0, M-$ некоторая константа;

2) матричный вес $W_{\varepsilon}^{2}(x):=\Phi(x-i \varepsilon)\left(w_{-}^{*}(x-i \varepsilon) w_{-}(x-i \varepsilon)\right)^{-1} \Phi^{*}(x-i \varepsilon)$ удовлетворяет $\left(A_{2}\right)$-условию на $\mathbb{R}$.

Доказательства теорем 2.1, 2.1' отличаются лишь незначительными деталями и основаны на теореме 1.4 .

ДОКАЗАТЕЛЬСТво теОРЕмы $2.1^{\prime}$. Вычислим значение столбца $F(h, z)$ в том случае, когда

$$
h=\sum_{k=1}^{n} \alpha_{k}\left(I-\lambda B^{*}\right)^{-1} g_{k}^{\Theta}, \quad \alpha_{k} \in \mathbb{C}, \quad \alpha=\operatorname{col}\left(\alpha_{k}\right)_{k=1}^{n} .
$$

Поскольку

$$
\left(I-z B^{*}\right)^{-1}\left(I-\lambda B^{*}\right)^{-1}=(z-\lambda)^{-1}\left(z\left(I-z B^{*}\right)^{-1}-\lambda\left(I-\lambda B^{*}\right)^{-1}\right),
$$

учитывая (2.3), приходим к формуле

$$
\begin{aligned}
f_{k}(h, z) & =\sum_{j=1}^{n} \Psi_{k j}(z) \sum_{m=1}^{n} \alpha_{m}\left(\left(I-z B^{*}\right)^{-1}\left(I-\lambda B^{*}\right)^{-1} g_{m}^{\Theta}, f_{j}^{\Theta}\right) \\
& =\sum_{m=1}^{n} \sum_{j=1}^{n} \Psi_{k j}(z)(\lambda-z)^{-1}\left(\Phi_{j m}(z)-\Phi_{j m}(\lambda)\right) \alpha_{m},
\end{aligned}
$$

которую можно переписать в векторном виде:

$$
F(h, z)=(\lambda-z)^{-1} \Phi^{-1}(z)(\Phi(z)-\Phi(\lambda)) \alpha .
$$

Вычислим теперь значение $F(\varphi, z)$ для вектора $\varphi$ вида

$$
\varphi=\int_{\mathbb{R}-i \varepsilon} \sum_{k=1}^{n}\left(I-\lambda B^{*}\right)^{-1} g_{k}^{\Theta} l_{k}(\lambda) d \lambda
$$


где вектор-функция $l(\lambda):=\operatorname{col}\left(l_{k}(\lambda)\right)_{k=1}^{n}$ удовлетворяет условию

$$
\int_{\mathbb{R}-i \varepsilon}\left\|w_{-}(z) l(z)\right\|_{\mathbb{C}^{n}}^{2} d z<\infty
$$

Заметим, что из теоремы 1.4 следует включение $\varphi \in \mathcal{K}_{\Theta}$. Учитывая формулу $(2.5)$, получим

$$
F(\varphi, z)=\int_{\mathbb{R}-i \varepsilon} F\left(\sum_{k=1}^{n}\left(I-\lambda B^{*}\right)^{-1} g_{k}^{\Theta} l_{k}(\lambda), z\right) d \lambda=\Phi^{-1}(z) \int_{\mathbb{R}-i \varepsilon} \frac{\Phi(z)-\Phi(\lambda)}{\lambda-z} l(\lambda) d \lambda .
$$

Напомним также, что из теоремы 1.4 следует оценка

$$
\|\varphi\|_{\mathcal{K}_{\Theta}}^{2} \leqslant M_{1} \int_{\mathbb{R}-i \varepsilon}\left\|w_{-}(z) l(z)\right\|_{\mathbb{C}^{n}}^{2} d z
$$

поэтому если справедлива оценка из условия 1) теоремы, то, рассматривая ее на векторах вида (2.6), получим

$$
\begin{aligned}
\int_{\mathbb{R}-i \varepsilon}\left\|w_{-}(z) F(\varphi, z)\right\|_{\mathbb{C}^{n}}^{2} d z & =\int_{\mathbb{R}-i \varepsilon}\left\|w_{-}(z) \Phi^{-1}(z) \int_{\mathbb{R}-i \varepsilon} \frac{\Phi(z)-\Phi(\lambda)}{\lambda-z} l(\lambda) d \lambda\right\|^{2} d z \\
& \leqslant M\|\varphi\|^{2} \leqslant M M_{1} \int_{\mathbb{R}-i \varepsilon}\left\|w_{-}(z) l(z)\right\|_{\mathbb{C}^{n}}^{2} d z .
\end{aligned}
$$

В терминах преобразования Гильберта $\mathcal{H}$ полученное неравенство переписывается в виде

$$
\begin{gathered}
\int_{\mathbb{R}}\left\|w_{-}(x-i \varepsilon) \mathcal{H} l(u-i \varepsilon)-w_{-}(x-i \varepsilon) \Phi^{-1}(x-i \varepsilon) \mathcal{H} \Phi(u-i \varepsilon) l(u-i \varepsilon)\right\|^{2} d x \\
\leqslant M_{2} \int_{\mathbb{R}}\left\|w_{-}(x-i \varepsilon) l(x-i \varepsilon)\right\|^{2} d x
\end{gathered}
$$

Известно [12], что каждый вес $w_{\varepsilon}^{2}(x):=w_{-}^{*}(x-i \varepsilon) w_{-}(x-i \varepsilon), \varepsilon>0, x \in \mathbb{R}$, также принадлежит классу $\mathcal{M}_{n}^{2}$, коль скоро $w^{2} \in \mathcal{M}_{n}^{2}$. Поэтому из теоремы В следует, что

$$
\int_{\mathbb{R}}\left\|w_{-}(x-i \varepsilon) \mathcal{H} l(u-i \varepsilon)\right\|_{\mathbb{C}^{n}}^{2} d x \leqslant M_{3} \int_{\mathbb{R}}\left\|w_{-}(x-i \varepsilon) l(x-i \varepsilon)\right\|^{2} d x,
$$

и, стало быть, из (2.7) выводим неравенство

$$
\int_{\mathbb{R}}\left\|w_{-}(x-i \varepsilon) \Phi^{-1}(x-i \varepsilon) \mathcal{H} \Phi(u-i \varepsilon) l(u-i \varepsilon)\right\|^{2} d x \leqslant M_{4} \int_{\mathbb{R}}\left\|w_{-}(x-i \varepsilon) l(u-i \varepsilon)\right\|^{2} d x .
$$

Для вектор-функции $f(u):=\Phi(u-i \varepsilon) l(u-i \varepsilon)$ имеем

$$
\int_{\mathbb{R}}\left\|w_{-}(u-i \varepsilon) \Phi^{-1}(u-i \varepsilon) f(u)\right\|^{2} d u=\int_{\mathbb{R}}\left\|w_{-}(u-i \varepsilon) l(u-i \varepsilon)\right\|^{2} d u<\infty .
$$

Неравенство (2.8) перепишем в виде

$$
\int_{\mathbb{R}}\|Q(x) \mathcal{H} f(u)\|^{2} d x \leqslant M_{4} \int_{\mathbb{R}}\|Q(x) f(x)\|^{2} d x,
$$

где введено обозначение $Q(x)=w_{-}(x-i \varepsilon) \Phi^{-1}(x-i \varepsilon)$. Из теоремы В следует, что вес $Q^{*}(x) Q(x)$, а вместе с ним и вес

$$
W_{\varepsilon}^{2}(x)=\Phi(x-i \varepsilon)\left(w_{-}^{*}(x-i \varepsilon) w_{-}(x-i \varepsilon)\right)^{-1} \Phi^{*}(x-i \varepsilon)
$$

принадлежат классу $\mathcal{M}_{n}^{2}$. 
Обратно, пусть $W_{\varepsilon}^{2} \in \mathcal{M}_{n}^{2}$. Тогда имеет место оценка (2.8), а значит, справедлива оценка (2.7). При доказательстве первой части теоремы было показано, что неравенство (2.7) можно переписать в виде

$$
\int_{\mathbb{R}-i \varepsilon}\left\|w_{-}(z) F(\varphi, z)\right\|_{\mathbb{C}^{n}}^{2} d z \leqslant M \int_{\mathbb{R}-i \varepsilon}\left\|w_{-}(z) l(z)\right\|^{2} d z,
$$

где векторы $\varphi$ и $l$ связаны равенством (2.6). Рассмотрим последнее неравенство на подпространстве $L$ из второй части теоремы 1.4. Тогда $\varphi$ - произвольный вектор модельного пространства $\mathcal{K}_{\Theta}$ и имеет место оценка

$$
\int_{\mathbb{R}-i \varepsilon}\left\|w_{-}(z) l(z)\right\|^{2} d z \leqslant M_{1}\|\varphi\|_{\mathcal{K}_{\Theta}}^{2} .
$$

Из двух последних неравенств вытекает утверждение 1). Теорема доказана.

Лемма 2.1. Если вес $W_{\varepsilon}^{2}(x)=\Phi(x+i \varepsilon)\left(w_{+}^{*}(x+i \varepsilon) w_{+}(x+i \varepsilon)\right)^{-1} \Phi^{*}(x+i \varepsilon)$, $\varepsilon>0$, принадлежит классу $\mathcal{M}_{n}^{2}$ и матрица-функиия

$$
W_{+}(z):=\Phi(z+i \varepsilon) w_{+}^{-1}(z+i \varepsilon) \Theta(z+i \varepsilon), \quad z \in \mathbb{C}_{+},
$$

является внешней ${ }^{3}$ в $\mathbb{C}_{+}$, то имеет место оценка снизу

$$
\delta\|h\|_{\mathcal{K}_{\Theta}}^{2} \leqslant \int_{\mathbb{R}+i \varepsilon}\left\|w_{+}(z) F(h, z)\right\|^{2} d z, \quad h \in \mathcal{K}_{\Theta},
$$

где $\delta$ - некоторая положительная константа.

ДокАЗАТЕльство. Вернемся к доказательству теоремы 1.4 для случая прямой $\mathbb{R}+i \varepsilon, \varepsilon>0$. Было доказано, что если в $(1.18)$ положить $l(z)=$ $-\frac{1}{2 \pi i} w_{+}^{-1}(z) \varphi_{0}(z-2 \varepsilon i)$, то интеграл $(1.18)$ равен $\varphi_{0}, \varphi_{0} \in \mathcal{K}_{\Theta}$. Другими словами, доказано представление

$$
\varphi=\int_{\mathbb{R}+i \varepsilon} \sum_{k=1}^{n}\left(I-z B^{*}\right)^{-1} g_{k}^{\Theta} l_{k}(z) d z, \quad l(z)=-\frac{1}{2 \pi i} w_{+}^{-1}(z) \varphi(z-2 \varepsilon i),
$$

для любой функции $\varphi \in \mathcal{K}_{\Theta}$. Поэтому с учетом $(2.5)$ приходим к равенству

$$
\begin{aligned}
F(\varphi, z) & =\int_{\mathbb{R}+i \varepsilon} F\left(\sum_{k=1}^{n}\left(I-\lambda B^{*}\right)^{-1} g_{k}^{\Theta} l_{k}(\lambda), z\right) d \lambda \\
& =\frac{\Phi^{-1}(z)}{2 \pi i} \int_{\mathbb{R}+i \varepsilon} \frac{\Phi(z)-\Phi(\lambda)}{z-\lambda} w_{+}^{-1}(\lambda) \varphi(\lambda-2 \varepsilon i) d \lambda, \quad \varphi \in \mathcal{K}_{\Theta},
\end{aligned}
$$

из которого следует, что

$$
\begin{aligned}
w_{+}(x+i \varepsilon) F(\varphi, x+i \varepsilon) & =-\frac{1}{2} w_{+}(x+i \varepsilon) \mathcal{H} w_{+}^{-1}(u+i \varepsilon) \varphi(u-i \varepsilon) \\
& +\frac{1}{2} w_{+}(x+i \varepsilon) \Phi^{-1}(x+i \varepsilon) \mathcal{H} \Phi(u+i \varepsilon) w_{+}^{-1}(u+i \varepsilon) \varphi(u-i \varepsilon) .
\end{aligned}
$$

Заметим, что функция $f(u):=w_{+}^{-1}(u+i \varepsilon) \varphi(u-i \varepsilon)$ принадлежит пространству $L_{2}^{n}\left(v^{2}\right)$, где вес $v^{2}(u):=w_{+}^{*}(u+i \varepsilon) w_{+}(u+i \varepsilon)$ принадлежит классу $\mathcal{M}_{n}^{2}$. Более того, поскольку функция $w_{+}(z+i \varepsilon)$ внешняя в $\mathbb{C}_{+}$и

$$
w_{+}^{*}(z+i \varepsilon) f(z)=\varphi(z-\varepsilon i) \in H_{+}^{2}\left(\mathbb{C}^{n}\right),
$$

\footnotetext{
${ }^{3}$ То есть $\operatorname{det} W_{+}(z)-$ внешняя функция в $\mathbb{C}_{+}$.
} 
то $f \in H_{+}^{2}\left(\mathbb{C}^{n}, v^{2}\right)$ (см. п. 1.1). Тогда в силу теоремы С справедливо равенство $\mathcal{H}=2 \mathcal{P}_{+}-I$ и, следовательно,

$$
-\frac{1}{2} w_{+}(x+i \varepsilon) \mathcal{H} w_{+}^{-1}(u+i \varepsilon) \varphi(u-i \varepsilon)=-\frac{1}{2} \varphi(x-i \varepsilon) .
$$

Аналогично, поскольку вес

$$
W_{\varepsilon}^{-2}(x)=\left(w_{+}(u+i \varepsilon) \Phi^{-1}(u+i \varepsilon)\right)^{*}\left(w_{+}(u+i \varepsilon) \Phi^{-1}(u+i \varepsilon)\right)
$$

принадлежит классу $\mathcal{M}_{n}^{2}$, снова можно использовать формулу $\mathcal{H}=2 \mathcal{P}_{+}-I$, где $\mathcal{P}_{+}-$проектор из $L_{2}\left(W_{\varepsilon}^{-2}\right)$ на $H_{+}^{2}\left(\mathbb{C}^{n}, W_{\varepsilon}^{-2}\right)$. Таким образом, учитывая формулу для матрицы-функции $W_{+}$, приходим к равенству

$w_{+}(x+i \varepsilon) F(\varphi, x+i \varepsilon)=-\varphi(x-i \varepsilon)+\Theta(x+i \varepsilon) W_{+}^{-1}(x) \mathcal{P}_{+} W_{+}(u) \Theta^{-1}(u+i \varepsilon) \varphi(u-i \varepsilon)$.

Из того, что $\Theta \in J_{n}$, следует формула $\Theta^{-1}(z)=\Theta^{*}(\bar{z}), z \in \mathbb{C}$, и имеет место оценка $\|\Theta(z)\| \leqslant \exp \{a|\operatorname{Im} z|\}$, где $a-$ экспоненциальный тип $\Theta$ [17]. Поэтому существует константа $C$ такая, что

$$
\|\Theta(x+i \varepsilon)\| \leqslant C, \quad\left\|\Theta^{-1}(x+i \varepsilon)\right\| \leqslant C, \quad x \in \mathbb{R} .
$$

Таким образом, поскольку $W_{\varepsilon}^{2} \in \mathcal{M}_{n}^{2}$, оператор

$$
\begin{aligned}
& W_{+}^{-1}(x) \mathcal{P}_{+} W_{+}(u) h(u) \\
& \quad=\Theta^{-1}(x+i \varepsilon) w_{+}(x+i \varepsilon) \Phi^{-1}(x+i \varepsilon) \mathcal{P}_{+} \Phi(u+i \varepsilon) w_{+}^{-1}(u+i \varepsilon) \Theta(u+i \varepsilon) h(u)
\end{aligned}
$$

ограничен в пространстве $L_{2}^{n}(\mathbb{R})$. Тогда в силу утверждения 2) теоремы C вектор

$$
h_{+}(x):=W_{+}^{-1}(x) \mathcal{P}_{+} W(u) \Theta^{-1}(u+i \varepsilon) \varphi(u-i \varepsilon), \quad \varphi \in \mathcal{K}_{\Theta},
$$

принадлежит пространству $H_{+}^{2}\left(\mathbb{C}^{n}\right)$ для любого $\varphi \in \mathcal{K}_{\Theta}$.

Докажем теперь, что слагаемые в правой части (2.9) ортогональны, т. е.

$$
\left(\varphi(x-i \varepsilon), \Theta(x+i \varepsilon) h_{+}(x)\right)_{L_{2}^{n}(\mathbb{R})}=0, \quad \varphi \in \mathcal{K}_{\Theta} .
$$

Заметим, что множество функций $\left\{f_{+}(x+i \varepsilon): f_{+} \in H_{+}^{2}\left(\mathbb{C}^{n}\right)\right\}$ всюду плотно в классе Харди $H_{+}^{2}\left(\mathbb{C}^{n}\right)$. Поэтому достаточно доказать $(2.10)$ для случая $h_{+}(x)=f_{+}(x+i \varepsilon), f_{+} \in H_{+}^{2}\left(\mathbb{C}^{n}\right)$. Поскольку $\varphi(z), z \in \mathbb{C}$, и $\Theta(z) f_{+}(z), z \in \mathbb{C}_{+}$, можно представить интегралом Фурье-Лапласа, из унитарности преобразования Фурье следует, что

$$
\left(\varphi(x-i \varepsilon), \Theta(x+i \varepsilon) f_{+}(x+i \varepsilon)\right)=\left(\varphi(x), \Theta(x) f_{+}(x)\right)=0, \quad \varphi \in \mathcal{K}_{\Theta} .
$$

Таким образом, из (2.9) вытекает оценка снизу

$$
\int_{\mathbb{R}}\left\|w_{+}(x+i \varepsilon) F(\varphi, x+i \varepsilon)\right\|_{\mathbb{C}^{n}}^{2} d x \geqslant \int_{\mathbb{R}}\|\varphi(x-i \varepsilon)\|^{2} d x \geqslant m \int_{\mathbb{R}}\|\varphi(x)\|^{2} d x,
$$

что и требовалось доказать.

Напомним, что для веса $v^{2} \in \mathcal{M}_{n}^{2}$ матрицы-функции $v_{-}, v_{+}$входят в факторизацию (1.1). Матрица $v_{-}(x-i 0) v_{+}^{-1}(x+i 0)$ унитарна почти при всех $x \in \mathbb{R}$ и порождает оператор Теплица

$$
T f:=\mathbb{P}_{+} v_{-}(x-i 0) v_{+}^{-1}(x+i 0) f(x), \quad f \in H_{+}^{2}\left(\mathbb{C}^{n}\right),
$$

где $\mathbb{P}_{+}-$ортопроектор из $L_{2}^{n}(\mathbb{R})$ на $H_{+}^{2}\left(\mathbb{C}^{n}\right)$. 
Лемма 2.2. Существует константа $\delta>0$ такая, что $\|T f\| \geqslant \delta\|f\| \partial л я$ вcex $f \in H_{+}^{2}\left(\mathbb{C}^{n}\right)$.

ДокАЗАТЕЛЬСтво. Поскольку $v^{2} \in \mathcal{M}_{n}^{2}$, оператор $L g:=v_{+} \mathbb{P}_{+} v_{-}^{-1} g, g \in$ $H_{+}^{2}\left(\mathbb{C}^{n}\right)$, ограничен в $H_{+}^{2}\left(\mathbb{C}^{n}\right)$ [12]. Поэтому оператор

$$
T L g=\mathbb{P}_{+} v_{-} v_{+}^{-1} v_{+} \mathbb{P}_{+} v_{-}^{-1} g=\mathbb{P}_{+} v_{-} \mathbb{P}_{+} v_{-}^{-1} g
$$

также ограничен в векторном классе Харди. Для всюду плотного в $H_{+}^{2}\left(\mathbb{C}^{n}\right)$ множества векторов

$$
g=c(x-\lambda)^{-1}, \quad c \in \mathbb{C}^{n}, \quad \operatorname{Im} \lambda<0,
$$

можем вычислить

$$
\mathbb{P}_{+} v_{-} \mathbb{P}_{+} v_{-}^{-1} g=\mathbb{P}_{+} v_{-}\left(v_{-}^{-1}(\lambda) c(x-\lambda)^{-1}\right)=v_{-}(\lambda) v_{-}^{-1}(\lambda) c(x-\lambda)^{-1}=g,
$$

т. е. $\mathbb{P}_{+} v_{-} \mathbb{P}_{+} v_{-}^{-1} g=g$ теперь для всех $g \in H_{+}^{2}\left(\mathbb{C}^{n}\right)$. Легко видеть также, что $\operatorname{Ker} T=\{0\}$. Поэтому из равенства $T f=g$ следует, что $f=L g$ и, стало быть,

$$
\|f\|=\|L g\| \leqslant\|L\|\|g\|=\|L\|\|T f\| .
$$

Лемма доказана.

2.2. Теперь мы можем сформулировать первый результат о полугруппах в модельном случае. Напомним, что рассматриваются операторы вида

$$
K h=B^{*} h+\sum_{k=1}^{n}\left(h, f_{k}^{\Theta}\right) g_{k}^{\Theta}, \quad h \in \mathcal{K}_{\Theta},
$$

которым отвечают пары $\Theta \in J_{n}, w^{2} \in \mathcal{M}_{n}^{2}$. Фредгольмов спектр оператора $K$ совпадает с множеством корней целой функции $\Delta(z)=\operatorname{det} \Phi(z)$, где элементы целой матрицы-функции $\Phi(z)$ определяются равенствами

$$
\Phi_{k j}(z)=\delta_{k j}-z\left(\left(I-z B^{*}\right)^{-1} g_{j}^{\Theta}, f_{k}^{\Theta}\right) .
$$

ТЕОРема 2.2. Пусть при некотором $\varepsilon \geqslant 0$ выполняются следующие условия:

1) корни функции $\Delta$ лежат под прямой $\mathbb{R}+i \varepsilon$;

2) $\lim \sup _{y \rightarrow+\infty} y^{-1} \log |\operatorname{det}(\Phi(i y) \Theta(i y))|=0$;

3) вес $W_{\varepsilon}^{2}(x)=\Phi(x+i \varepsilon)\left(w_{+}^{*}(x+i \varepsilon) w_{+}(x+i \varepsilon)\right)^{-1} \Phi^{*}(x+i \varepsilon)$ удовлетворяет матричному условию $\left(A_{2}\right)$ на $\mathbb{R}$.

Тогда справедливы утверждения:

а) $\operatorname{Ker} K=\operatorname{Ker} K^{*}=\{0\}$ u оператор $A:=K^{-1}$ есть $w$-возмущение операmopa $A_{0}:=\left(B^{*}\right)^{-1}$ класса $\Sigma^{(\exp )}$;

b) полугруппа $U_{-}(t)=\exp \{-i A t\}, t \geqslant 0$, принадлежит классу $C_{0}$, причем ее экспоненииальный тип не превосходит $\varepsilon$.

ДокАЗАтельство. Шаг 1. Докажем, что матрица-функция

$$
W_{+}(z)=\Phi(z+i \varepsilon) w_{+}^{-1}(z+i \varepsilon) \Theta(z+i \varepsilon), \quad z \in \mathbb{C}_{+},
$$

является внешней в $\mathbb{C}_{+}$. Из формулы $(2.11)$ следует, что $k$-я строка матрицы $z^{-1}\left(E_{n}-\Phi(z)\right)$ имеет вид $\mathcal{B}\left(z, f_{k}^{\Theta}\right), 1 \leqslant k \leqslant n$. В силу теоремы 1.2 элементы 
матрицы $z^{-1}\left(E_{n}-\Phi(z)\right) w_{+}^{-1}(z) \Theta(z)$ принадлежат классу $H_{+}^{2}$. Поэтому элементы матрицы $(z+i)^{-1} W_{+}(z), z \in \mathbb{C}_{+}$также принадлежат $H_{+}^{2}$. Это свойство матрицы $W_{+}(z)$ обеспечивает наличие факторизации

$$
W_{+}(z)=W_{e}(z) V(z), \quad z \in \mathbb{C}_{+},
$$

где $W_{e}-$ внешняя, а $V$ - внутренняя матрицы-функции в $\mathbb{C}_{+}[13]$. Переходя к определителям, получим равенство

$$
\operatorname{det}(\Phi(z+i \varepsilon) \Theta(z+i \varepsilon))=Q(z) \operatorname{det} V(z), \quad z \in \mathbb{C}_{+},
$$

где $Q(z)$ - некоторая внешняя функция. Поскольку функция слева в последнем равенстве аналитична на вещественной оси, то $\operatorname{det} V(z)=e^{i \alpha z}, \alpha \geqslant 0$ [8]. Легко видеть, что из условия 2 ) теоремы следует, что $\alpha=0$, т. е. матрица-функция $W_{+}$ внешняя в $\mathbb{C}_{+}$.

Шаг 2. Из теоремы 2.1 и леммы 2.1 вытекает двусторонняя оценка

$$
m\|h\|^{2} \leqslant \int_{\mathbb{R}+i \varepsilon}\left\|w_{+}(z) F(h, z)\right\|^{2} d z \leqslant M\|h\|^{2}, \quad h \in \mathcal{K}_{\Theta} .
$$

Поскольку вес $w_{+}^{*}(x+i \varepsilon) w_{+}(x+i \varepsilon)$ принадлежит $\mathcal{M}_{n}^{2}[12]$, то в силу $(1.1)$ имеем

$$
w_{+}^{*}(x+i \varepsilon) w_{+}(x+i \varepsilon) \stackrel{\text { п.в. }}{=}\left(w_{-}^{\varepsilon}(x-i 0)\right)^{*} w_{-}^{\varepsilon}(x-i 0), \quad x \in \mathbb{R},
$$

где $w_{-}^{\varepsilon}-$ внешняя матрица-функция в $\mathbb{C}_{-}$. Далее, в процессе доказательства леммы 2.1 установлено, что координаты вектор-функции $w_{+}(z) F(h, z)$ принадлежат классу Харди $H_{+}^{2}$ в области $\operatorname{Im} z>\varepsilon$. Поэтому из леммы 2.2 выводится оценка

$$
\begin{aligned}
& \delta \int_{\mathbb{R}+i \varepsilon}\left\|w_{+}(z) F(h, z)\right\|^{2} d z \\
& \quad \leqslant \int_{\mathbb{R}}\left\|\mathbb{P}_{+} w_{-}^{\varepsilon}(x-i 0) w_{+}^{-1}(x+i \varepsilon) w_{+}(x+i \varepsilon) F(h, x+i \varepsilon)\right\|^{2} d x \\
& \quad=\int_{\mathbb{R}}\left\|\mathbb{P}_{+} w_{-}^{\varepsilon}(x-i 0) F(h, x+i \varepsilon)\right\|^{2} d x .
\end{aligned}
$$

Кроме того, из определения $w_{-}^{\varepsilon}(x-i 0)$ вытекает, что

$$
\int_{\mathbb{R}}\left\|\mathbb{P}_{+} w_{-}^{\varepsilon}(x-i 0) F(h, x+i \varepsilon)\right\|^{2} d x \leqslant \delta \int_{\mathbb{R}+i \varepsilon}\left\|w_{+}(z) F(h, z)\right\|^{2} d z .
$$

Таким образом, из (2.12) следует двойное неравенство

$$
\delta_{1}\|h\|^{2} \leqslant \int_{\mathbb{R}}\left\|\mathbb{P}_{+} w_{-}^{\varepsilon}(x-i 0) F(h, x+i \varepsilon)\right\|^{2} d x \leqslant \delta_{2}\|h\|^{2} .
$$

Шаг 3. Докажем справедливость формулы

$$
\operatorname{col}\left((I-z K)^{-1} h, f_{k}^{\Theta}\right)_{k=1}^{n}=F(h, z), \quad h \in \mathcal{K}_{\Theta} .
$$

В самом деле, из формулы (2.2) легко следует, что

$$
(I-z K)^{-1} h=\left(I-z B^{*}\right)^{-1} h+z \sum_{k=1}^{n} f_{k}(h, z)\left(I-z B^{*}\right)^{-1} g_{k}^{\Theta},
$$


поэтому с учетом формул (2.3) найдем

$$
\begin{aligned}
& \left((I-z K)^{-1} h, f_{s}^{\Theta}\right)=\left(\left(I-z B^{*}\right)^{-1} h, f_{s}^{\Theta}\right)+z \sum_{k=1}^{n} f_{k}(h, z)\left(\left(I-z B^{*}\right)^{-1} g_{k}^{\Theta}, f_{s}^{\Theta}\right) \\
& =\left(\left(I-z B^{*}\right)^{-1} h, f_{s}\right)+\sum_{k=1}^{n} f_{k}(h, z)\left(\delta_{s k}-\Phi_{s k}(z)\right)=\left(\left(I-z B^{*}\right)^{-1} h, f_{s}\right) \\
& \quad-\sum_{k=1}^{n} f_{k}(h, z) \Phi_{s k}(z)+f_{s}(h, z)=f_{s}(h, z), \quad 1 \leqslant s \leqslant n
\end{aligned}
$$

что и доказывает (2.14).

В силу (2.13) оператор

$$
S h:=\mathbb{P}_{+} w_{-}^{\varepsilon}(x-i 0) F(h, x+i \varepsilon), \quad h \in \mathcal{K}_{\Theta},
$$

есть изоморфизм пространства $\mathcal{K}_{\Theta}$ на некоторое подпространство $\mathcal{L}$ пространства Харди $H_{+}^{2}\left(\mathbb{C}^{n}\right)$. Предполагая, что $\lambda^{-1} \notin \sigma(K)$, вычислим

$$
S K(I-\lambda K)^{-1} h=\mathbb{P}_{+} w_{-}^{\varepsilon}(x-i 0) F\left(K(I-\lambda K)^{-1} h, x+i \varepsilon\right) .
$$

Из (2.14) вытекает соотношение

$$
\begin{aligned}
& F\left(K(I-\lambda K)^{-1} h, x+i \varepsilon\right)=\operatorname{col}\left\{\left(K(I-\lambda K)^{-1}(I-(x+i \varepsilon) K)^{-1} h, f_{k}^{\Theta}\right)\right\}_{k=1}^{n} \\
& =\frac{\operatorname{col}\left\{\left((I-(x+i \varepsilon) K)^{-1} h, f_{k}^{\Theta}\right)\right\}_{k=1}^{n}-\operatorname{col}\left\{\left((I-\lambda K)^{-1} h, f_{k}^{\Theta}\right)\right\}_{k=1}^{n}}{x+i \varepsilon-\lambda}, \quad h \in \mathcal{K}_{\Theta},
\end{aligned}
$$

при выводе которого была использована формула

$$
(I-z K)^{-1}-(I-\lambda K)^{-1}=(z-\lambda)(I-\lambda K)^{-1} K(I-z K)^{-1}, \quad z^{-1}, \lambda^{-1} \notin \sigma(K) .
$$

Возвращаясь к (2.16), с учетом (2.14) получим

$$
S K(I-\lambda K)^{-1} h=\mathbb{P}_{+} w_{-}^{\varepsilon}(x-i 0) \frac{F(h, x+i \varepsilon)-F(h, \lambda)}{x-(\lambda-i \varepsilon)}, \quad h \in \mathcal{K}_{\Theta} .
$$

Предположим, что $\operatorname{Im}(\lambda-i \varepsilon)>0$. Тогда, учитывая (2.15), можем продолжить вычисления:

$$
\begin{aligned}
S K(I-\lambda K)^{-1} h & =\mathbb{P}_{+} w_{-}^{\varepsilon}(x-i 0) \frac{F(h, x+i \varepsilon)}{x-(\lambda-i \varepsilon)} \\
& =\mathbb{P}_{+} \frac{\mathbb{P}_{+} w_{-}^{\varepsilon}(x-i 0) F(h, x+i \varepsilon)}{x-(\lambda-i \varepsilon)}+\mathbb{P}_{+} \frac{\mathbb{P}_{-} w_{-}^{\varepsilon}(x-i 0) F(h, x+i \varepsilon)}{x-(\lambda-i \varepsilon)} \\
& =\mathbb{P}_{+} \frac{(S h)(x)}{x-(\lambda-i \varepsilon)} .
\end{aligned}
$$

Полагая здесь $\mu=\lambda-i \varepsilon$, найдем

$$
S K(I-(\mu+i \varepsilon) K)^{-1} h=\mathbb{P}_{+} \frac{(S h)(x)}{x-\mu}=\frac{(S h)(x)-(S h)(\mu)}{x-\mu}, \quad \operatorname{Im} \mu>0 .
$$

Таким образом, подпространство $\mathcal{L}$ пространства $H_{+}^{2}\left(\mathbb{C}^{n}\right)$ инвариантно относительно действия операторов

$$
\left(R_{\mu} \varphi\right)(x)=\frac{\varphi(x)-\varphi(\mu)}{x-\mu}, \quad \varphi \in \mathcal{L}, \quad \operatorname{Im} \mu>0 .
$$

4 Серия математическая, т. 75, № 2 
Отсюда вытекает [13], [24] существование мероморфной в $\mathbb{C}$ и внутренней в $\mathbb{C}_{+}$ матрицы-функции $\Pi(z)$ такой, что $\mathcal{L}=H_{+}^{2}\left(\mathbb{C}^{n}\right) \ominus \Pi(z) H_{+}^{2}\left(\mathbb{C}^{n}\right)$. Поскольку $(i \varepsilon)^{-1} \notin \sigma(K)$, из $(2.17)$ вытекает, что каждая вектор-функция $\varphi \in \mathcal{L}$ аналитична в окрестности нуля. Из соотношения (2.17) при $\mu=0$ вытекает равенство

$$
S K(I-i \varepsilon K)^{-1} h=L S h, \quad h \in \mathcal{K}_{\Theta},
$$

в котором диссипативный оператор $L$ задается формулой

$$
(L \varphi)(z)=z^{-1}(\varphi(z)-\varphi(0)), \quad \varphi \in \mathcal{L}, \quad \mathcal{L}=H_{+}^{2}\left(\mathbb{C}^{n}\right) \ominus \Pi(z) H_{+}^{2}\left(\mathbb{C}^{n}\right) .
$$

Отсюда выводится, что $\operatorname{Ker} K=\operatorname{Ker} K^{*}=\{0\}$ и, как это следует из теоремы 1.1 , оператор $A:=K^{-1}$ есть $w$-возмущение оператора $A_{0}:=\left(B^{*}\right)^{-1}$. Теперь равенство (2.18) перепишется в виде

$$
(A-i \varepsilon I)^{-1}=S^{-1} L S .
$$

Таким образом, полугруппа $U_{-}(t)$ принадлежит классу $C_{0}$, и $U_{-}(t)=e^{\varepsilon t} S^{-1} \times$ $\exp \left\{-i L^{-1} t\right\} S$, причем полугруппа $\exp \left\{-i L^{-1} t\right\}$ сжимающая. Теорема доказана.

СлЕДСТВИЕ 2.1. Если выполнены условия 1)-3) теоремы 2.2, то существует мероморфная в $\mathbb{C}$ и внутренняя в $\mathbb{C}_{+}$матрица-функиия П такая, что опеpатор $(A-i \varepsilon I)^{-1}$ подобен модельному оператору $L$, который задается формулами (2.19).

Заметим, что из общей теории функциональных моделей [10], [14] следует, что матрица $\Pi(z)$ является характеристической матрицей-функцией оператоpa $L$. Мы в состоянии получить достаточно полную информацию о П $(z)$, однако это выходит за рамки настоящей статьи.

2.3. В этом пункте мы рассмотрим задачу о необходимости условий теоремы 2.2. Теперь мы предполагаем, что для оператора $K$ вида $(2.1)$ выполняются условия $\operatorname{Ker} K=\operatorname{Ker} K^{*}=\{0\}$ и, стало быть, существует оператор $A=K^{-1}$. Итак, предположим, что полугруппа $U_{-}(t)=\exp \{-i A t\}$ принадлежит классу $C_{0}$. Поскольку $\sigma(A)$ совпадает с множеством корней функции $\Delta(z)=\operatorname{det} \Phi(z)$, где $\Phi$ определяется формулой (2.11), очевидно, что условие 1$)$ теоремы 2.2 является необходимым для принадлежности $U_{-}(t)$ классу $C_{0}$. Докажем теперь необходимость условия 2).

Лемма 2.3. Если полугруппа $U_{-}(t)$ принадлежит классу $C_{0}$, то

$$
\limsup _{y \rightarrow+\infty} y^{-1} \log |\operatorname{det}(\Phi(i y) \Theta(i y))|=0 .
$$

ДокАзАтельство. Шаг 1. Как и при доказательстве теоремы 2.2 (шаг 1), получаем внешне-внутреннюю факторизацию

$$
\Phi(z+i \varepsilon) w_{+}^{-1}(z+i \varepsilon) \Theta(z+i \varepsilon)=W_{e}(z) V(z), \quad z \in \mathbb{C}_{+},
$$

где $W_{e}$ - внешняя, $V$ - внутренняя матрицы-функции в $\mathbb{C}_{+}$. Отметим, что в этой формуле $\varepsilon>\max \{0, \omega\}$, где $\omega-$ экспоненциальный тип полугруппы $U_{-}(t)$. Было установлено, что из (2.21) вытекает равенство $\operatorname{det} V(z)=e^{i \alpha z}$, $\alpha \geqslant 0, z \in \mathbb{C}_{+}$. Матрица-функция $V\left(i(1+\lambda)(1-\lambda)^{-1}\right),|\lambda|<1$, является характеристической для некоторого сжатия $T$ в некотором гильбертовом 
пространстве $\mathfrak{H}$ [14]. Известно [14], [25] что детерминант характеристической матрицы-функции не может аналитически продолжаться на спектр сжатия. В случае полуплоскости заключаем, что матрица $V(z)$ в $(2.21)$ является целой матрицей экспоненциального типа [17]. Предположим теперь, что (2.20) не имеет места. Тогда в факторизации (2.21) матрица-функция $V$ отлична от унитарной константы.

Шаг 2. Если полугруппа $U_{-}(t)$ принадлежит классу $C_{0}$ и имеет тип $\omega$, то в области $\operatorname{Im} z>\omega$ имеет место следующее представление резольвенты:

$$
\int_{0}^{\infty} U_{-}(t) e^{i z t} d t=-i(A-z I)^{-1} .
$$

Отметим, что формулу (2.14) можно переписать в виде

$$
\operatorname{col}\left(A(A-z I)^{-1} h, f_{k}^{\Theta}\right)_{k=1}^{n}=F(h, z),
$$

поэтому координаты вектор-функции $F(h, z+i \varepsilon)\left(z-z_{0}\right)^{-1}, z \in \mathbb{C}_{+}, z_{0} \in \mathbb{C}_{-}$, являются ограниченными в $\mathbb{C}_{+}$и, кроме того, в силу теоремы Винера-Пэли они принадлежат $H_{+}^{2}$. В частном случае $h=\sum_{k=1}^{n} C_{k}\left(I-z_{0} B^{*}\right)^{-1} g_{k}^{\Theta}$ из формулы (2.5) следует равенство

$$
\frac{F(h, z+i \varepsilon)}{z_{0}-z}=\left(z_{0}-z\right)^{-2} \Phi^{-1}(z+i \varepsilon)\left(\Phi(z+i \varepsilon)-\Phi\left(z_{0}\right)\right) C, \quad C=\operatorname{col}\left(C_{k}\right)_{k=1}^{n},
$$

где $C$ - произвольный вектор из $\mathbb{C}^{n}$. Отсюда выводится, что все элементы матрицы-функции $\left(z_{0}-z\right)^{-2} \Phi^{-1}(z+i \varepsilon)$ ограничены в $\mathbb{C}_{+} \cdot$ Из $(2.21)$ следует, что

$$
\frac{w_{+}(z+i \varepsilon)}{z-z_{0}} \frac{\Phi^{-1}(z+i \varepsilon)}{\left(z-z_{0}\right)^{2}} \frac{W_{e}(z)}{z-z_{0}}=\frac{\Theta(z+i \varepsilon) V^{-1}(z)}{\left(z-z_{0}\right)^{4}}, \quad z \in \mathbb{C}_{+} .
$$

Из теоремы А вытекает, что элементы матрицы $w_{+}(z+i \varepsilon)\left(z-z_{0}\right)^{-1}$ принадлежат пространству $H_{+}^{2}$. Из теоремы 1.2 и равенства (2.21) следует, что элементы матрицы $W_{e}(z)\left(z-z_{0}\right)^{-1}$ также принадлежат $H_{+}^{2}$. Поэтому элементы матрицы $\Theta(z+i \varepsilon) V^{-1}(z)\left(z-z_{0}\right)^{-4}$ принадлежат классу $H_{+}^{1}$. Таким образом, при каждом $\lambda \in \mathbb{C}_{-}$имеем

$$
\int_{\mathbb{R}} \frac{\Theta(x+i \varepsilon) V^{-1}(x)}{(x-\lambda)^{3}\left(x-z_{0}\right)} d x=0 .
$$

Поскольку элементы $\Theta(x+i \varepsilon) V^{-1}(x)\left(x-z_{0}\right)^{-1}$ принадлежат $L_{2}(\mathbb{R})$, отсюда заключаем, что они принадлежат классу Харди $H_{+}^{2}$. Поэтому имеет место факторизация

$$
\Theta(x+i \varepsilon) V^{-1}(x)=Q(z) \Pi(z), \quad z \in \mathbb{C}_{+},
$$

где $Q-$ внешняя, $\Pi$ - внутренняя матрицы-функции. Ограниченная в $\mathbb{C}_{+}$ матрица-функция $\Theta(x+i \varepsilon)$ также факторизуется:

$$
\Theta(z+i \varepsilon)=Q_{1}(z) \Pi_{1}(z), \quad z \in \mathbb{C}_{+},
$$

где $Q_{1}$ - ограниченная внешняя, $\Pi_{1}$ - внутренняя матрицы-функции. Из двух последних равенств следует, что

$$
Q(z) \Pi(z) V(z)=Q_{1}(z) \Pi_{1}(z), \quad z \in \mathbb{C}_{+} .
$$

В силу единственности факторизации можем считать, что $Q=Q_{1}$, стало быть, из $(2.22)$ вытекает ограниченность матрицы $\Theta(z+i \varepsilon) V^{-1}(z)$ в $\mathbb{C}_{+}$. Поскольку 
матрица $\Theta(z+i \varepsilon) V^{-1}(z)$ ограничена в любой горизонтальной полосе конечной ширины [17], то $\left\|Q(z) V^{-1}(z-i \varepsilon)\right\| \leqslant C, z \in \mathbb{C}_{+}$. Поэтому справедлива факторизация

$$
\Theta(z) V^{-1}(z-i \varepsilon)=V_{1}(z) W_{1}(z), \quad z \in \mathbb{C}_{+},
$$

где $V_{1}$ - внутренняя, $W_{1}$ - внешняя матрицы-функции. Поскольку детерминант целой внутренней функции в $\mathbb{C}_{+}$имеет вид $u e^{i \alpha z}, \alpha>0$, где $|u|=1$, из последнего равенства следует, что $\operatorname{det} W_{1}(z)=v e^{i \gamma z},|v|=1, \gamma \in \mathbb{R}$. Так как $W_{1}$ внешняя, то $\gamma=0$ и, стало быть, $W_{1}(z) \equiv A$ - постоянная матрица. Далее, из (2.23) вытекает представление

$$
\Theta(z)=V_{1}(z) A V(z-i \varepsilon)=V_{1}(z) V_{2}(z) W_{2}(z), \quad z \in \mathbb{C}_{+},
$$

где $V_{1}, V_{2}$ - целые внутренние функции, $W_{2}$ - внешняя. Из единственности внешне-внутренней факторизации следует, что $W_{2}(z)$ - постоянная матрица, и поэтому из предыдущего равенства выводим представление

$$
\Theta(z)=V_{1}(z) U_{2}(z), \quad V_{1}, U_{2} \in J_{n} .
$$

Шаг 3. Из $(2.21)$ и $(2.23)$ (с учетом того, что $\left.W_{1}(z) \equiv A\right)$ находим:

$$
\begin{aligned}
& \frac{\Phi(z+i \varepsilon)}{z-z_{0}} w_{+}^{-1}(z+i \varepsilon) V_{1}(z+i \varepsilon)=\frac{W_{e}(z)}{z-z_{0}} V(z) \Theta^{-1}(z+i \varepsilon) V_{1}(z+i \varepsilon) \\
& \quad=\frac{W_{e}(z)}{z-z_{0}} A^{-1} V_{1}^{-1}(z+i \varepsilon) V_{1}(z+i \varepsilon)=\frac{W_{e}(z)}{z-z_{0}} A^{-1}, \quad z \in \mathbb{C}_{+}, \quad \operatorname{Im} z_{0}<0 .
\end{aligned}
$$

Таким образом, элементы матрицы

$$
F(z):=\frac{E_{n}-\Phi(z+i \varepsilon)}{z+i \varepsilon} w_{+}^{-1}(z+i \varepsilon) V_{1}(z+i \varepsilon), \quad z \in \mathbb{C}_{+},
$$

принадлежат классу Харди $H_{+}^{2}$. Из теоремы 1.2 вытекает, что, поскольку матрица-функция $\Theta^{-1}(z) V_{1}(z)$ ограничена в полосе $0 \leqslant \operatorname{Im} z \leqslant \varepsilon$, элементы матрицы

$$
z^{-1}\left(E_{n}-\Phi(z)\right) w_{+}^{-1}(z) V_{1}(z)=z^{-1}\left(E_{n}-\Phi(z)\right) w_{+}^{-1}(z) \Theta(z) \Theta^{-1}(z) V_{1}(z)
$$

принадлежат классу Харди в этой полосе. В итоге приходим к следующему важному выводу: элементы матрицы

$$
F_{1}(z):=z^{-1}\left(E_{n}-\Phi(z)\right) w_{+}^{-1}(z) V_{1}(z), \quad z \in \mathbb{C}_{+},
$$

принадлежат классу Харди $H_{+}^{2}$.

Шаг 4. Поскольку строки матрицы $z^{-1}\left(E_{n}-\Phi(z)\right)$ лежат в образе преобразования $\mathcal{B}(z, h)$, из теоремы 1.2 следует, что

$$
z^{-1}\left(E_{n}-\Phi(z)\right) w_{-}^{-1}(z) \in{ }^{*} H_{-}^{2}\left(\mathbb{C}^{n}\right) .
$$

Заметим, что из (2.24) вытекает включение $\mathcal{K}_{V_{1}} \subseteq \mathcal{K}_{\Theta}$ и $\mathcal{K}_{V_{1}}$ инвариантно относительно оператора $B$ вида (1.3). Введем оператор $B_{1}:=B \mid \mathcal{K}_{V_{1}}$ и векторы $g_{k}^{1}$ с помощью формул

$$
\left(I+i B_{1}^{*}\right)^{-1} g_{k}^{1}=\mathbb{P}_{V_{1}} \frac{w_{+}^{k}(x+i 0)}{x+i}, \quad 1 \leqslant k \leqslant n,
$$


и обозначим соответствующее преобразование $(1.8)$ через $\mathcal{B}_{1}(z, h), h \in \mathcal{K}_{V_{1}}$. Поскольку элементы матрицы $F_{1}(z)$ принадлежат классу Харди $H_{+}^{2}$, строки матрицы $z^{-1}\left(E_{n}-\Phi(z)\right)$ принадлежат образу $\mathcal{B}_{1}(z, h)$, т. е.

$$
z^{-1}\left(\delta_{j k}-\Phi_{j k}(z)\right)=\left(\left(I-z B_{1}^{*}\right)^{-1} g_{k}^{1}, f_{j}^{1}\right)_{\mathcal{K}_{V_{1}}}, \quad j=1,2, \ldots, n,
$$

где $f_{j}^{1} \in \mathcal{K}_{V_{1}}, k=1,2, \ldots, n$. Учитывая лемму 1.1, для $z \in \mathbb{C}_{-}$получим

$$
\begin{aligned}
\left(\left(I-z B_{1}^{*}\right)^{-1} g_{k}^{1}, f_{j}^{1}\right)_{\mathcal{K}_{V_{1}}} & =\left(\mathbb{P}_{V_{1}} \frac{w_{+}^{k}(x+i 0)}{x-z}, f_{j}^{1}\right)_{\mathcal{K}_{V_{1}}}=\left(\mathbb{P}_{\Theta} \frac{w_{+}^{k}(x+i 0)}{x-z}, f_{j}^{1}\right)_{\mathcal{K}_{\Theta}} \\
& =\left(\left(I-z B^{*}\right)^{-1} g_{k}^{\Theta}, f_{j}^{1}\right)_{\mathcal{K}_{\Theta}},
\end{aligned}
$$

где векторы $g_{k}^{\Theta}$ определяются равенствами (1.6). Сравнивая полученную формулу с (2.11), приходим к равенствам

$$
\left(\left(I-z B^{*}\right)^{-1} g_{k}^{\Theta}, f_{j}^{\Theta}\right)_{\mathcal{K}_{\Theta}}=\left(\left(I-z B^{*}\right)^{-1} g_{k}^{\Theta}, f_{j}^{1}\right)_{\mathcal{K}_{\Theta}}, \quad 1 \leqslant k, j \leqslant n,
$$

значит, в силу биективности $\mathcal{B}(z, h)$ имеем $f_{j}^{\Theta}=f_{j}^{1}$ для всех $j$. Таким образом, векторы $f_{j}^{\Theta}$ принадлежат $\mathcal{K}_{V_{1}}$, и поэтому подпространство $\mathcal{L}:=\mathcal{K}_{\Theta} \ominus \mathcal{K}_{V_{1}}$ инвариантно относительно оператора $K$. В самом деле, если $l \in \mathcal{L}$, то

$$
K l=B^{*} l+\sum_{j=1}^{n}\left(l, f_{j}^{\Theta}\right) g_{j}^{\Theta}=B^{*} l \in \mathcal{L},
$$

поскольку $\mathcal{K}_{V_{1}}$ инвариантно относительно $B$. Итак, $K\left|\mathcal{L}=B^{*}\right| \mathcal{L}$. Поэтому если полугруппа $U_{-}(t)=\exp \left\{-i K^{-1} t\right\}$ принадлежит классу $C_{0}$, то $\exp \left\{-i\left(B^{*} \mid \mathcal{L}\right)^{-1} t\right\}$ также принадлежит этому классу. Однако это невозможно, поскольку полугруппа $\exp \left\{i\left(B^{*}\right)^{-1} t\right\}, t \geqslant 0$, является сжимающей и нильпотентной. Поэтому имеет место равенство (2.20). Лемма доказана.

Обсудим теперь необходимость условия 3) из формулировки теоремы 2.2. Сначала приведем следующий результат.

Лемма 2.4. Пусть $\Theta \in J_{n}$ такова, что при некотором $\delta>0$ матрицафункиия $e^{-i \delta z} \Theta(z)$ ограничена в $\mathbb{C}_{+}$. Тогда на каждой прямой $\operatorname{Im} z=b, b \neq 0$, имеет место оценка снизу

$$
\left\|\sum_{k=1}^{n} C_{k}\left(I-z B^{*}\right)^{-1} g_{k}^{\Theta}\right\|^{2} \geqslant q(b)\|\widetilde{w}(z) C\|^{2}, \quad C=\operatorname{col}\left(C_{k}\right)_{k=1}^{n},
$$

где $\widetilde{w}(z)=w_{+}(z), z \in \mathbb{C}_{+}, u \widetilde{w}(z)=w_{-}(z), z \in \mathbb{C}_{-}$.

ДокАЗАТЕЛЬСтво. В силу ограниченности матрицы-функции $e^{-i \delta z} \Theta(z)$ приходим к выводу, что элементы матрицы

$$
x^{-1}\left(e^{-i \delta x}-1\right) E_{n} w_{+}^{-1}(x+i 0) \Theta(x)=w_{+}^{-1}(x+i 0) x^{-1}\left(e^{-i \delta x} \Theta(x)-\Theta(x)\right)
$$

принадлежат классу Харди $H_{+}^{2}$. Согласно теореме А элементы матрицы $x^{-1}\left(e^{-i \delta x}-1\right) E_{n} w_{-}^{-1}(x-i 0)$ принадлежат $H_{-}^{2}$, а в силу теоремы 1.2 строки матрицы $z^{-1}\left(e^{-i \delta z}-1\right) E_{n}$ принадлежат образу преобразования $\mathcal{B}(z, h)$, т. е.

$$
\operatorname{row}\left(z^{-1}\left(e^{-i \delta z}-1\right) \delta_{k j}\right)_{j=1}^{n}=\operatorname{row}\left(\left(I-z B^{*}\right)^{-1} g_{j}^{\Theta}, f_{k}^{0}\right)_{j=1}^{n}, \quad f_{k}^{0} \in \mathcal{K}_{\Theta},
$$


где $k=1,2, \ldots, n$. Таким образом, матрица-функция $\Phi^{0}(z):=e^{-i \delta z} E_{n}$ представляется в виде

$$
\Phi_{k j}^{0}(z)=\delta_{k j}-z\left(\left(I-z B^{*}\right)^{-1} g_{j}^{\Theta}, f_{k}^{0}\right), \quad 1 \leqslant k, j \leqslant n .
$$

Рассмотрим в пространстве $\mathcal{K}_{\Theta}$ оператор $K^{0}$ вида $(2.1)$ с векторами $f_{k}^{\Theta}=f_{k}^{0}$. Отвечающая ему в силу формулы $(2.3)$ матрица-функция равна $\Phi^{0}(z)$. Далее, вес

$$
W_{0}^{2}(x):=\Phi^{0}(x)\left(w_{+}^{*}(x+i 0) w_{+}(x+i 0)\right)^{-1}\left(\Phi^{0}(x)\right)^{*}=w^{-2}(x)
$$

принадлежит классу $\mathcal{M}_{n}^{2}$. Поэтому в силу теоремы 2.1

$$
\int_{\mathbb{R}}\left\|w_{+}(x+i 0) F^{0}(h, x)\right\|_{\mathbb{C}^{n}}^{2} d x \leqslant M\|h\|^{2}
$$

для всех $h \in \mathcal{K}_{\Theta}$. Напомним, что вектор-функция $F^{0}(h, x)$ определяется формулами $(2.3),(2.4)$. Полагая здесь $h=\sum_{k=1}^{n} C_{k}\left(I-z B^{*}\right)^{-1} g_{k}^{\Theta}$ и применяя формулу (2.5), найдем

$$
\begin{gathered}
F^{0}(h, x)=(z-x)^{-1}\left(\Phi^{0}(x)\right)^{-1}\left(\Phi^{0}(x)-\Phi^{0}(z)\right) C=(z-x)^{-1}\left(1-e^{i \delta x} e^{-i z \delta}\right) C, \\
C=\operatorname{col}\left(C_{k}\right)_{k=1}^{n} .
\end{gathered}
$$

Поэтому приходим к оценке снизу

$$
M\left\|\sum_{k=1}^{n} C_{k}\left(I-z B^{*}\right)^{-1} g_{k}\right\|^{2} \geqslant \int_{\mathbb{R}}\left\|\left(1-e^{i x \delta} e^{-i z \delta}\right)(x-z)^{-1} w_{+}(x+i 0) C\right\|^{2} d x .
$$

Пусть $z \in \mathbb{C}_{+}$и $z=a+b i$. Тогда

$$
\begin{aligned}
& M\left\|\sum_{k=1}^{n} C_{k}\left(I-z B^{*}\right)^{-1} g_{k}\right\|^{2} \geqslant\left(1-e^{b \delta}\right)^{2} \int_{\mathbb{R}}\left\|\frac{w_{+}(x+i 0) C}{x-z}\right\|^{2} d x \\
& \geqslant\left(1-e^{b \delta}\right)^{2} \int_{\mathbb{R}}\left\|\mathbb{P}_{-} \frac{w_{+}(x+i 0) C}{x-z}\right\|^{2} d x=\left(1-e^{b \delta}\right)^{2} \int_{\mathbb{R}} \frac{\left\|w_{+}(z) C\right\|^{2}}{|x-z|^{2}} d x \\
& \geqslant \frac{\left(1-e^{b \delta}\right)^{2}}{2 b}\left\|w_{+}(z) C\right\|^{2}
\end{aligned}
$$

где $\mathbb{P}_{-}-$ортпроектор из $L_{2}^{n}(\mathbb{R})$ на $H_{-}^{2}\left(\mathbb{C}^{n}\right)$.

Если $z \in \mathbb{C}_{-}$, то в силу $(1.1)$ в неравенстве $(2.26)$ заменим $w_{+}(x+i 0)$ на $w_{-}(x-i 0)$. После этого необходимо повторить изложенные рассуждения с заменой $\mathbb{P}_{-}$на $\mathbb{P}_{+}$. Лемма доказана.

ЗАмечАниЕ 2.1. Оценка снизу (2.25), вообще говоря, не имеет места для произвольной матрицы-функции $\Theta \in J_{n}$. В самом деле, если $w^{2}(x) \equiv E_{n}$, то из (1.6) следуют формулы

$$
g_{k}^{\Theta}=\left(I+i B^{*}\right) \mathbb{P}_{\Theta} \frac{e_{k}}{x+i},
$$

где $e_{k}-$ стандартные орты пространства $\mathbb{C}^{n}$. Учитывая формулу $\mathbb{P}_{\Theta}=\Theta \mathbb{P}_{-} \Theta^{*}$, получим

$$
\begin{aligned}
\mathbb{P}_{\Theta} \frac{e_{k}}{x+i} & =\Theta \mathbb{P}_{-} \frac{\Theta^{*}(x) e_{k}}{x+i}=\Theta(x) \frac{\Theta^{*}(x)-\Theta^{*}(i)}{x+i} e_{k} \\
& =\frac{\left(E_{n}-\Theta(x) \Theta^{*}(i)\right)}{x+i} e_{k}, \quad 1 \leqslant k \leqslant n .
\end{aligned}
$$


Из (1.4) следует, что

$$
\left(I+i B^{*}\right) h=\frac{(z+i) h(z)-i \Theta(z) h(0)}{z} .
$$

В результате элементарных вычислений приходим к формулам

$$
g_{k}^{\Theta}=z^{-1}\left(E_{n}-\Theta(z)\right) e_{k}, \quad 1 \leqslant k \leqslant n .
$$

С другой стороны, из формул (1.3), (1.4) вытекает, что

$$
\begin{aligned}
\frac{B-B^{*}}{i} h & =i \frac{E_{n}-\Theta(z)}{z} h(0)=i \frac{E_{n}-\Theta(z)}{z} \sum_{k=1}^{n}\left(h(0), e_{k}\right) e_{k} \\
& =\sum_{k=1}^{n}\left(h(0), e_{k}\right) i g_{k}^{\Theta}=\frac{1}{2 \pi} \sum_{k=1}^{n}\left(h, i g_{k}^{\Theta}\right) i g_{k}^{\Theta}=\sum_{k=1}^{n}\left(h, \varphi_{k}\right) \varphi_{k}
\end{aligned}
$$

где введены обозначения $(\sqrt{2} \pi)^{-1} i g_{k}^{\Theta}=\varphi_{k}$. Элементарные вычисления показывают, что

$$
\Theta_{j k}(z)=\delta_{k j}+i z\left((I-z B)^{-1} \varphi_{k}, \varphi_{j}\right), \quad 1 \leqslant k, j \leqslant n .
$$

Поэтому можем воспользоваться известной формулой [17]

$$
-\left(\left(E_{n}-\Theta(\bar{z}) \Theta^{*}(\bar{z})\right) e_{k}, e_{j}\right)=\operatorname{Im} z\left(\left(I-z B^{*}\right)^{-1} \varphi_{k},\left(I-z B^{*}\right)^{-1} \varphi_{j}\right) .
$$

Таким образом, если $z \in \mathbb{C}_{+}$, то

$$
\begin{aligned}
\left\|\sum_{k=1}^{n} C_{k}\left(I-z B^{*}\right)^{-1} g_{k}^{\Theta}\right\|^{2} & =2 \pi(\operatorname{Im} z)^{-1}\left(\left(\Theta(\bar{z}) \Theta^{*}(\bar{z})-E_{n}\right) C, C\right) \\
& =2 \pi(\operatorname{Im} z)^{-1}\left(\left\|\Theta^{*}(\bar{z}) C\right\|^{2}-\|C\|^{2}\right) .
\end{aligned}
$$

Отметим, что $w_{+}(z) \equiv E_{n}$ в рассматриваемом случае и, следовательно, оценка (2.25) имеет вид

$$
\left\|\sum_{k=1}^{n} C_{k}\left(I-z B^{*}\right)^{-1} g_{k}^{\Theta}\right\|^{2} \geqslant q(b)\|C\|^{2}, \quad \operatorname{Im} z=b>0 .
$$

Поскольку $\Theta$ - произвольная функция класса $J_{n}$, ясно, что такая оценка, вообще говоря, места не имеет.

ЗАмечание 2.2. Для любых $\Theta \in J_{n}$ и $w^{2} \in \mathcal{M}_{n}^{2}$ на каждой прямой $\operatorname{Im} z=$ $b \neq 0$ имеет место неравенство

$$
\left\|\sum_{k=1}^{n} C_{k}\left(I-z B^{*}\right)^{-1} g_{k}^{\Theta}\right\|^{2} \leqslant q_{1}(b)\|\widetilde{w}(z) C\|^{2} .
$$

Мы не приводим доказательство этой оценки, так как в дальнейшем она не используется.

ЛЕмма 2.5. Пусть полугруппа $U_{-}(t)$ принадлежит классу $C_{0}$ и имеет экспоненииальный тип $\omega$. Если при некотором $\delta>0$ матрища-функиия $e^{-i z \delta} \Theta(z)$ ограничена в $\mathbb{C}_{+}$, то при каждом $\varepsilon>\max \{0, \omega\}$ выполняется условие 3) теоремы 2.2 . 
ДокАЗАТЕльство. Если полугруппа $U_{-}(t)$ принадлежит классу $C_{0}$, то в области $\operatorname{Im} z>\omega$ справедливо равенство

$$
-i(A-z I)^{-1}=\int_{0}^{\infty} e^{i z t} U_{-}(t) d t .
$$

Поэтому для каждого $h \in \mathcal{K}_{\Theta}$ имеем

$$
-i(A-(x+i \varepsilon) I)^{-1} h=\int_{0}^{\infty} e^{i x t} e^{-\varepsilon t} U_{-}(t) h d t .
$$

Считая, что $\varepsilon>\max \{0, \omega\}$, получим

$$
\int_{\mathbb{R}}\left\|(A-(x+i \varepsilon) I)^{-1} h\right\|^{2} d x=2 \pi \int_{0}^{\infty}\left\|e^{-\varepsilon t} U_{-}(t) h\right\|^{2} d t \leqslant M\|h\|^{2} .
$$

Из формулы (2.2) следует, что

$$
(A-z I)^{-1} h=\left(\left(B^{*}\right)^{-1}-z I\right)^{-1} h+\sum_{k=1}^{n} f_{k}(h, z)\left(I-z B^{*}\right)^{-1} g_{k}^{\Theta} .
$$

Неограниченный оператор $\left(B^{*}\right)^{-1}$ является диссипативным, и поэтому полугруппа $\exp \left\{i\left(B^{*}\right)^{-1} t\right\}, t \geqslant 0$, является сжимающей и нильпотентной. Таким образом, для любого $\varepsilon>\max \{0, \omega\}$ имеем

$$
\int_{\mathbb{R}}\left\|B^{*}\left(I-(x+i \varepsilon) B^{*}\right)^{-1} h\right\|^{2} d x \leqslant M_{1}\|h\|^{2}, \quad h \in \mathcal{K}_{\Theta} .
$$

Итак, из трех последних формул вытекает, что

$$
\int_{\mathbb{R}+i \varepsilon}\left\|\sum_{k=1}^{n} f_{k}(z, h)\left(I-z B^{*}\right)^{-1} g_{k}^{\Theta}\right\|^{2} d z \leqslant M_{2}\|h\|^{2}, \quad h \in \mathcal{K}_{\Theta} .
$$

В силу (2.4) вектор $\operatorname{col}\left(f_{k}(z, h)\right)_{k=1}^{n}$ равен $F(z, h)$, и если применить лемму 2.4 , то найдем

$$
q(\varepsilon)\left\|w_{+}(z) F(z, h)\right\|^{2} \leqslant\left\|\sum_{k=1}^{n} f_{k}(z, h)\left(I-z B^{*}\right)^{-1} g_{k}^{\Theta}\right\|^{2}, \quad \operatorname{Im} z=\varepsilon .
$$

Поэтому

$$
\int_{\mathbb{R}+i \varepsilon}\left\|w_{+}(z) F(z, h)\right\|^{2} d z \leqslant M_{3}\|h\|^{2}, \quad h \in \mathcal{K}_{\Theta},
$$

и остается применить теорему 2.1. Лемма доказана.

2.4. Сформулируем теперь один из основных результатов настоящей статьи. Напомним, что плотно заданный неограниченный оператор $A$ в сепарабельном гильбертовом пространстве $\mathfrak{H}$ называется $w$-возмущением оператора $A_{0} \in \Sigma^{(\exp )}$, если

$$
A^{-1} h=A_{0}^{-1} h+\sum_{k=1}^{n}\left(h, f_{k}\right) g_{k}, \quad h \in \mathfrak{H},
$$

и существует вес $w^{2} \in \mathcal{M}_{n}^{2}$ такой, что строка

$$
\mathcal{A}_{0}(z, h)=\operatorname{row}\left\{\left(A_{0}\left(A_{0}-z I\right)^{-1} g_{k}, h\right)\right\}_{k=1}^{n}
$$


обладает свойствами:

1) $\left\|\mathcal{A}_{0}(x, h) w^{-1}(x)\right\|$ принадлежит $L_{2}(\mathbb{R})$ при каждом $h \in \mathcal{H}$;

2) имеет место оценка

$$
m\|h\|^{2} \leqslant \int_{\mathbb{R}}\left\|\mathcal{A}_{0}(x, h) w^{-1}(x)\right\|^{2} d x, \quad h \in \mathfrak{H} .
$$

Далее, оператору $A$ отвечает единственная матрица-функция $\Theta \in J_{n}$, которая определяется из условий:

a) $\Theta$ - левый делитель $e^{i a z} E_{n}$, где $a-$ экспоненциальный тип $\left(A_{0}-z I\right)^{-1}$;

b) координаты строки $\mathcal{A}_{0}(x, h) w_{+}^{-1}(x+i 0) \Theta(x)$ принадлежат классу $H_{+}^{2}$ для каждого $h \in \mathfrak{H}$;

c) если $\Theta_{1}$ - левый делитель $e^{i a z} E_{n}$, для которого выполняется условие b), то $\Theta_{1}=\Theta V$, где $V \in J_{n}$.

Напомним, что $\sigma(A)$ совпадает с множеством корней определителя $\Delta(z):=$ $\operatorname{det} \Phi(z)$, где элементы матрицы $\Phi$ определяются формулами

$$
\Phi_{k j}(z)=\delta_{k j}-z\left(A_{0}\left(A_{0}-z I\right)^{-1} g_{j}, f_{k}\right) .
$$

И наконец, системы векторов $\left\{f_{k}\right\}_{k=1}^{n},\left\{g_{k}\right\}_{k=1}^{n}$ предполагаются линейно независимыми (см. введение).

В формулировке следующей теоремы фигурирует величина $-i \operatorname{Sp} \Theta^{\prime}(0), \Theta \in$ $J_{n}$. Поскольку каждая матрица $\Theta \in J_{n}$ является характеристической матрицей-функцией некоторого диссипативного оператора $\stackrel{\circ}{B}$, то в силу формулы $\left(1.5^{\prime}\right)$ получим $-i \mathrm{Sp} \Theta^{\prime}(0)=\sum_{k=1}^{n}\left\|\varphi_{k}\right\|^{2}>0$. Далее, существует $\Theta_{1} \in J_{n}$ такая, что $\Theta(z) \Theta_{1}(z)=e^{i a z} E_{n}$, где $a-$ экспоненциальный тип $\Theta(z)$. Тогда

$$
n a=-\left.i \operatorname{Sp}\left(\Theta(z) \Theta_{1}(x)\right)^{\prime}\right|_{z=0}=-i \operatorname{Sp} \Theta^{\prime}(0)-i \operatorname{Sp} \Theta_{1}^{\prime}(0) \geqslant-i \operatorname{Sp} \Theta^{\prime}(0) .
$$

Таким образом, $0<-i \operatorname{Sp} \Theta^{\prime}(0) \leqslant n a$.

Tеорема 2.3. Пусть $A-w$-возмущение оператора $A_{0} \in \Sigma^{(\exp )}$, которому отвечает пара $w^{2} \in \mathcal{M}_{n}^{2}, \Theta \in J_{n}$. Пусть при некотором $\varepsilon \geqslant 0$ выполняются условия:

1) $\sup _{\lambda_{k} \in \Lambda} \operatorname{Im} \lambda_{k}<\varepsilon$, где $\Lambda$ - множество корней $\Delta(z)$;

2) $\lim \sup _{y \rightarrow+\infty} y^{-1} \log |\Delta(i y)|=-i \operatorname{Sp} \Theta^{\prime}(0)$;

3) матричный вес

$$
W_{\varepsilon}^{2}(x):=\Phi(x+i \varepsilon)\left(w_{+}^{*}(x+i \varepsilon) w_{+}(x+i \varepsilon)\right)^{-1} \Phi^{*}(x+i \varepsilon)
$$

удовлетворяет условию $\left(A_{2}\right)$ на $\mathbb{R}$.

Тогда полугруппа $U_{-}(t):=\exp \{-i A t\}, t \geqslant 0$, принадлежит классу $C_{0}$, причем ее экспоненииальный тип не превосходит $\varepsilon$.

Обратно, если $U_{-}(t) \in C_{0}$ и ее тип равен $\omega$, то при любом $\varepsilon>\omega$ выполняются условия 1), 2). Кроме того, если при некотором $\delta>0$ матрица-функция $e^{-i \delta z} \Theta(z)$ ограничена в $\mathbb{C}_{+}$, то при любом $\varepsilon>\max \{0, \omega\}$ выполняется условие 3).

ДокАЗАТЕЛЬСтво. В силу теоремы 1.5 существует изоморфизм $Q$ пространства $\mathfrak{H}$ на $\mathcal{K}_{\Theta}$ такой, что

$$
B^{*}=Q A_{0}^{-1} Q^{-1}, \quad Q g_{k}=g_{k}^{\Theta}, \quad k=1,2, \ldots, n,
$$

где $B^{*}$ - модельный оператор в $\mathcal{K}_{\Theta}$, векторы $g_{k}^{\Theta}$ определяются равенствами вида (1.6). Поэтому $Q A^{-1} Q^{-1}=K$, где $K$ - оператор вида (2.1) при 
$f_{k}^{\Theta}=\left(Q^{*}\right)^{-1} f_{k}, 1 \leqslant k \leqslant n$. Применим теорему 2.2 к оператору $K$. Для этого вычислим элементы матрицы-функции $\Phi$ вида $(2.27)$ :

$$
\begin{aligned}
\Phi_{k j}(z) & =\delta_{k j}-z\left(\left(I-z A_{0}^{-1}\right)^{-1} g_{j}, f_{k}\right)=\delta_{k j}-z\left(Q^{-1}\left(I-z B^{*}\right)^{-1} Q g_{j}, f_{k}\right) \\
& =\delta_{k j}-z\left(\left(I-z B^{*}\right)^{-1} g_{j}^{\Theta}, f_{k}^{\Theta}\right) \mathcal{K}_{\Theta} .
\end{aligned}
$$

Таким образом, если доказать эквивалентность условия

$$
\limsup _{y \rightarrow \infty} y^{-1} \log |\operatorname{det}(\Phi(i y) \Theta(i y))|=0
$$

условию 2), то первая часть теоремы будет следовать из теоремы 2.2 .

Действительно, так как $\operatorname{det} \Theta(z)=e^{i p z}, p>0$, то (2.28) равносильно условию

$$
\limsup _{y \rightarrow \infty} y^{-1} \log |\Delta(i y)|=p \text {. }
$$

Для вычисления $p$ воспользуемся известной формулой [26]:

$$
\frac{d}{d z} \log \operatorname{det} \Theta(z)=\operatorname{Sp}\left(\Theta^{-1}(z) \frac{d \Theta(z)}{d z}\right) .
$$

Отсюда вытекает, что правая часть последнего равенства не зависит от $z$, и поэтому

$$
i p=\left.\operatorname{Sp}\left(\Theta^{-1}(z) \Theta^{\prime}(z)\right)\right|_{z=0}=\operatorname{Sp} \Theta^{\prime}(0) .
$$

Доказательство второй части теоремы с помощью теоремы 1.5 снова сводится к рассмотрению случая оператора $A=K^{-1}$, где $K$ задается формулой (2.1). Необходимость условия 1) очевидна. В лемме 2.3 доказана необходимость условия (2.28) и, тем самым, условия 2). И наконец, необходимость условия 3) в случае ограниченности в $\mathbb{C}_{+}$матрицы-функции $e^{-i \delta z} \Theta(z)$ доказана в лемме 2.4. Теорема доказана.

СлеДСтвиЕ 2.2. Пусть $A-w$-возмущение ранга $n$ оператора $A_{0}$ класса $\Sigma^{(\exp )}$. Если при некотором $\varepsilon>0$ выполняются условия 1)-3) теоремы 2.3, то существует мероморфная в $\mathbb{C}$ и внутренняя в $\mathbb{C}_{+}$матрица-функиия П такая, что оператор $(A-i \varepsilon I)^{-1}$ подобен модельному оператору

$$
(L \varphi)(z):=z^{-1}(\varphi(z)-\varphi(0))
$$

в пространстве $\mathcal{L}=H_{+}^{2}\left(\mathbb{C}^{n}\right) \ominus \Pi(z) H_{+}^{2}\left(\mathbb{C}^{n}\right)$.

Отметим, что этот результат вытекает из теоремы 1.5 и следствия 2.1 .

\section{§ 3. Описание генераторов \\ сильно непрерывных полугрупп $U_{+}(t)=\exp \{i A t\}$ и некоторые дополнительные сведения}

3.1. Как и раньше, теорема 1.5 сводит задачу описания генераторов сильно непрерывных полугрупп $U_{+}(t)=\exp \{i A t\}$ к описанию генераторов вида $A=K^{-1}$, где $K$ - произвольный оператор вида $(2.1)$ в пространстве $\mathcal{K}_{\Theta}$. Сначала рассмотрим случай, когда $\Theta(z)=e^{i a z} E_{n}, w^{2} \in \mathcal{M}_{n}^{2}$. Другими словами, рассмотрим оператор

$$
K_{a} h:=B_{a}^{*} h+\sum_{k=1}^{n}\left(h, f_{k}^{a}\right) g_{k}^{a}, \quad h \in \mathcal{K}_{a},
$$


где $f_{k}^{a}$ - произвольные векторы из $\mathcal{K}_{a}$, векторы $g_{k}^{a}$ определяются равенствами (1.6) в случае $\Theta(z)=e^{i a z} E_{n}$. Фредгольмов спектр оператора $K_{a}$ совпадает с множеством корней функции $\Delta_{a}(z):=\operatorname{det} \Phi_{a}(z)$, где элементы матрицы $\Phi_{a}$ находятся по формуле

$$
\Phi_{a}^{k j}(z)=\delta_{k j}-z\left(\left(I-z B_{a}^{*}\right)^{-1} g_{j}^{a}, f_{k}^{a}\right) .
$$

Докажем, что выбором векторов $f_{k}^{a}$ можно добиться того, чтобы $\Phi_{a}(z)=$ $e^{-i a z} E_{n}$. В самом деле, в силу теоремы А строки матрицы $z^{-1}\left(1-e^{-i a z}\right) E_{n}$ удовлетворяют условиям теоремы 1.2 в случае произвольного веса $w^{2}$ и $\Theta(z)=$ $e^{i a z} E_{n}$. Поэтому они входят в образ соответствующего преобразования $\mathcal{B}_{a}(z, h)$, т. е. существуют функции $f_{k}^{1} \in \mathcal{K}_{a}, 1 \leqslant k \leqslant n$, такие, что справедливо представление

$$
\left(e^{-i a z} E_{n}\right)_{k j}=\delta_{j k}-z\left(\left(I-z B_{a}^{*}\right)^{-1} g_{j}^{a}, f_{k}^{1}\right), \quad 1 \leqslant j \leqslant n .
$$

Таким образом, для оператора

$$
K_{a}^{1} h:=B_{a}^{*} h+\sum_{k=1}^{n}\left(h, f_{k}^{1}\right) g_{k}^{a}, \quad h \in \mathcal{K}_{a},
$$

матрица-функция $\Phi_{a}$ вида (3.2) равна $e^{-i a z} E_{n}$. Заметим, что вес

$$
\begin{aligned}
W_{\varepsilon}^{2}(x) & =e^{-i a(x-i \varepsilon)}\left(w_{-}^{*}(x-i \varepsilon) w_{-}(x-i \varepsilon)\right)^{-1} e^{i a(x+i \varepsilon)} \\
& =e^{-2 \varepsilon}\left(w_{-}^{*}(x-i \varepsilon) w_{-}(x-i \varepsilon)\right)^{-1}
\end{aligned}
$$

при любом $\varepsilon \geqslant 0$ принадлежит классу $\mathcal{M}_{n}^{2}$. Применяя к оператору (3.4) теорему $2.1^{\prime}$, заключаем, что

$$
\int_{\mathbb{R}-i \varepsilon}\left\|w_{-}(z) F_{1}(z, h)\right\|^{2} d z \leqslant M\|h\|^{2}, \quad h \in \mathcal{K}_{a},
$$

где в силу формул $(2.3),(2.4)$ имеем

$$
F_{1}(h, z)=e^{i a z} \operatorname{col}\left(\left(I-z B_{a}^{*}\right)^{-1} h, f_{j}^{1}\right)_{j=1}^{n}, \quad h \in \mathcal{K}_{a} .
$$

Лемма 3.1. Пусть вектори $f_{k}^{1}, 1 \leqslant k \leqslant n$, пространства $\mathcal{K}_{a}$ определяются формулами (3.3). Тогда вектор-функиия

$$
X(z, h):=\operatorname{col}\left(\left(I-z B_{a}^{*}\right)^{-1} h, f_{k}^{1}\right)_{k=1}^{n}
$$

обладает следующими свойствами:

1) для любого $\varepsilon>0$ найдутся константы $m, M$ такие, что

$$
m\|h\|^{2} \leqslant \int_{\mathbb{R}-i \varepsilon}\left\|w_{-}(z) X(z, h)\right\|^{2} d z \leqslant M\|h\|^{2}
$$

для всех $h \in \mathcal{K}_{a}$;

2) для каждого $h \in \mathcal{K}_{a}$ и каждого $\varepsilon>0$ имеем

$$
e^{i a x} v_{\varepsilon}(x+i 0) X(x-i \varepsilon, h) \in H_{+}^{2}\left(\mathbb{C}^{n}\right), \quad w_{-}(x-i \varepsilon) X(x-i \varepsilon, h) \in H_{-}^{2}\left(\mathbb{C}^{n}\right),
$$

где внешняя в $\mathbb{C}_{+}$матрица-функиия $v_{\varepsilon}$ взята из факторизации ${ }^{4}$

$$
w_{-}^{*}(x-i \varepsilon) w_{-}(x-i \varepsilon) \stackrel{n . \beta .}{=} v_{\varepsilon}^{*}(x+i 0) v_{\varepsilon}(x+i 0), \quad x \in \mathbb{R} .
$$

\footnotetext{
${ }^{4}$ Возможность такой факторизации вытекает из теоремы А.
} 
ДокАЗАтЕЛЬство. Согласно $F_{1}(z, h)=e^{i a z} X(z, h)$ имеем из (3.5) оценку сверху (3.6). Для оператора (3.4) матрица-функция $\Phi_{a}(z)$ равна $e^{-i a z} E_{n}$. Как и при доказательстве теоремы $2.1^{\prime}$, вычислим значение $F_{1}(h, z)$ на векторе вида (2.6). Мы получим формулу

$$
F_{1}(\varphi, z)=e^{i a z} \int_{\mathbb{R}-i \varepsilon} \frac{e^{-i a z}-e^{-i a \lambda}}{\lambda-z} l(\lambda) d \lambda,
$$

где функция $l(z)$ представляется в виде $(1.25)$, т. е.

$$
l(z)=w_{-}^{-1}(z) h(z), \quad h(z):=\int_{0}^{a} e^{i z t \overline{f(t)}} d t
$$

здесь $f$ - произвольная вектор-функция из $L_{2}^{n}(0, a)$. Из доказательства теоремы 1.4 (шаг 5) следует, что формулу (3.7) можно переписать следующим образом:

$$
F_{1}(S h, z)=e^{i a z} \int_{\mathbb{R}-i \varepsilon} \frac{e^{-i a z}-e^{-i a \lambda}}{\lambda-z} w_{-}^{-1}(\lambda) h(\lambda) d \lambda,
$$

где $h$ (в силу теоремы Пэли-Винера) пробегает $\mathcal{K}_{a}, S$ - некоторый автоморфизм пространства $\mathcal{K}_{a}$.

Если $z$ лежит в области $\operatorname{Im} z<-\varepsilon$, то

$$
F_{1}(S h, z)=2 \pi i w_{-}^{-1}(z) h(z)-2 \pi i \mathcal{P}_{-} w_{-}^{-1}(y-i \varepsilon) h(y-i \varepsilon) .
$$

Здесь был использован тот факт, что вектор-функция $e^{-i a x} w_{-}^{-1}(x-i \varepsilon) h(x-i \varepsilon)$ принадлежит классу Харди в $\mathbb{C}_{-}$с весом Макенхаупта $w_{-}^{*}(x-i \varepsilon) w_{-}(x-i \varepsilon)$. Устремляя $z$ к точкам прямой $\mathbb{R}-i \varepsilon$, найдем:

$(2 \pi i)^{-1} w_{-}(x-i \varepsilon) F_{1}(S h, x-i \varepsilon)=h(x-i \varepsilon)-w_{-}(x-i \varepsilon) \mathcal{P}_{-} w_{-}^{-1}(y-i \varepsilon) h(y-i \varepsilon)$.

Поскольку $h(x-i \varepsilon) \in H_{+}^{2}\left(\mathbb{C}^{n}\right)$, а второе слагаемое справа в $(3.8)$ принадлежит $H_{-}^{2}\left(\mathbb{C}^{n}\right)$ (теорема $\left.\mathrm{C}\right)$, имеем

$$
\int_{\mathbb{R}}\left\|w_{-}(x-i \varepsilon) F_{1}(S h, x-i \varepsilon)\right\|^{2} d x \geqslant m\|h(x-i \varepsilon)\|^{2} \geqslant m_{1}\|h\|^{2},
$$

т. е. оценка (3.6) доказана.

Умножая (3.8) на $e^{-i a x}$, приходим к выводу, что

$$
w_{-}(x-i \varepsilon) X(x-i \varepsilon, h) \in H_{-}^{2}\left(\mathbb{C}^{n}\right), \quad h \in \mathcal{K}_{a} .
$$

Далее, так как вес $v_{\varepsilon}^{2}(x):=w_{-}^{*}(x-i \varepsilon) w_{-}(x-i \varepsilon), x \in \mathbb{R}$, принадлежит классу $\mathcal{M}_{n}^{2}$, то вес ${ }^{t} v_{\varepsilon}^{-2}(x)$ (транспонированная матрица $\left.v_{\varepsilon}^{-2}(x)\right)$ также обладает этим свойством и факторизуется следующим образом:

$$
{ }^{t} v_{\varepsilon}^{-2}(x)=\left({ }^{t} v_{\varepsilon}^{-1}(x+i 0)\right) *\left({ }^{t} v_{\varepsilon}^{-1}(x+i 0)\right) .
$$

Оценка (3.6) означает, что каждая строка ${ }^{t} X(z-i \varepsilon, h), h \in \mathcal{K}_{a}$, удовлетворяет условиям теоремы 1.3 в случае веса ${ }^{t} v_{\varepsilon}^{-2}(x)$. Теперь из теоремы 1.2 в случае $\Theta(z)=e^{i a z} E_{n}$ следует включение

$$
{ }^{t} X(x-i \varepsilon, h){ }^{t} v_{\varepsilon}(x+i 0) e^{i a z} \in{ }^{*} H_{+}^{2}(\mathbb{C}), \quad h \in \mathcal{K}_{a},
$$

что и завершает доказательство леммы. 
Исследуем теперь произвольный оператор $K_{a}$ вида (3.1). Из формулы $(2.2)$ и соотношений (2.3) легко следует, что

$$
\left(I-z K_{a}\right)^{-1} h=\left(I-z B_{a}^{*}\right)^{-1} h+z \sum_{k=1}^{n} f_{k}(h, z)\left(I-z B^{*}\right)^{-1} g_{k}^{a} .
$$

Фиксируя $z_{0} \in \mathbb{C}$, введем в рассмотрение столбец

$$
\begin{aligned}
K_{0}(z, h) & :=\operatorname{col}\left(\left(I-z K_{a}\right)^{-1} h, f_{j}^{1}\left(z_{0}\right)\right)_{j=1}^{n}, \quad h \in \mathcal{K}_{a}, \\
f_{j}^{1}\left(z_{0}\right) & :=\left(I-\bar{z}_{0} B\right)^{-1} f_{j}^{1}, \quad 1 \leqslant j \leqslant n,
\end{aligned}
$$

где векторы $f_{j}^{1}$ определяются формулами (3.3). Имеем

$$
\begin{aligned}
& \left(\left(I-z B^{*}\right)^{-1} h, f_{j}^{1}\left(z_{0}\right)\right)=\left(\left(I-z B^{*}\right)^{-1}\left(I-z_{0} B^{*}\right)^{-1} h, f_{j}^{1}\right) \\
& \quad=\left(\frac{z\left(I-z B^{*}\right)^{-1} h-z_{0}\left(I-z_{0} B^{*}\right)^{-1} h}{z-z_{0}}, f_{j}^{1}\right)=\frac{z X(z, h)-z_{0} X\left(z_{0}, h\right)}{z-z_{0}},
\end{aligned}
$$

где $X(z, h)$ определено в формулировке леммы 3.1. Проводя аналогичные вычисления, с учетом (3.3) получим формулу

$$
\left(\left(I-z B^{*}\right)^{-1} g_{k}^{a}, f_{j}\left(z_{0}\right)\right)=\frac{\left(1-e^{-i a z}\right) \delta_{k j}-\left(1-e^{-i a z_{0}}\right) \delta_{k j}}{z-z_{0}}=\frac{e^{-i a z_{0}}-e^{-i a z}}{z-z_{0}} \delta_{k j} .
$$

Таким образом, учитывая обозначение (2.4), имеем

$$
K_{0}(z, h)=\frac{z X(z, h)-z_{0} X\left(z_{0}, h\right)}{z-z_{0}}+z \frac{e^{-i a z_{0}}-e^{-i a z}}{z-z_{0}} F(h, z) .
$$

Напомним, что вес $w_{-}^{*}(x-i \varepsilon) w_{-}(x-i \varepsilon)$ факторизуется следующим образом:

$$
w_{-}^{*}(x-i \varepsilon) w_{-}(x-i \varepsilon)=v_{\varepsilon}^{*}(x+i 0) v_{\varepsilon}(x+i 0), \quad \varepsilon>0,
$$

где $v_{\varepsilon}(z)-$ внешняя функция в области $\mathbb{C}_{+}$.

ЛЕмма 3.2. Пусть при некотором $\varepsilon>0$ выполняются следующие условия:

1) вес $W_{\varepsilon}^{2}(x)=\Phi_{a}(x-i \varepsilon)\left(w_{-}^{*}(x-i \varepsilon) w_{-}(x-i \varepsilon)\right)^{-1} \Phi_{a}^{*}(x-i \varepsilon), x \in \mathbb{R}$, принадлежит классу $\mathcal{M}_{n}^{2}$;

2) матрица-функиия $W_{-}(z):=\Phi_{a}(z-i \varepsilon) w_{-}^{-1}(z-i \varepsilon)$ внешняя в $\mathbb{C}_{-}$.

Тогда для столбца $K_{0}(z, h), \operatorname{Im} z_{0}<-\varepsilon$, построенного по оператору $K_{a}$ вида (3.1) с помощью формул (3.9), справедлива двусторонняя оценка

$$
m\|h\|^{2} \leqslant \int_{\mathbb{R}}\left\|v_{\varepsilon}(x+i 0) K_{0}(x-i \varepsilon, h)\right\|^{2} d x \leqslant M\|h\|^{2}, \quad h \in \mathcal{K}_{a} .
$$

ДокАЗАтЕЛЬство. Шаг 1. Сначала применим теорему $2.1^{\prime}$ для оператора вида (3.1). Из условия 1) следует, что

$$
\int_{\mathbb{R}}\left\|v_{\varepsilon}(x+i 0) F(h, x-i \varepsilon)\right\|^{2} d x=\int_{\mathbb{R}-i \varepsilon}\left\|w_{-}(z) F(h, z)\right\|^{2} d z \leqslant M\|h\|^{2}
$$

для всех $h \in \mathcal{K}_{a}$. Далее, из леммы 3.1 вытекает оценка

$$
\int_{\mathbb{R}}\left\|v_{\varepsilon}(x+i 0) X(x-i \varepsilon, h)\right\|^{2} d x=\int_{\mathbb{R}-i \varepsilon}\left\|w_{-}(z) X(z, h)\right\|^{2} d z \leqslant M\|h\|^{2} .
$$


После этого оценка сверху (3.11) тривиальным образом вытекает из формулы (3.10).

Шаг 2. Докажем справедливость неравенства

$$
m \int_{\mathbb{R}}\left\|\mathbb{P}_{-} v_{\varepsilon}(x+i 0) F(x-i \varepsilon, h)\right\|^{2} d x \leqslant \int_{\mathbb{R}}\left\|v_{\varepsilon}(x+i 0) K_{0}(x-i \varepsilon, h)\right\|^{2} d x,
$$

где $\mathbb{P}_{-}$- ортопроектор из $L_{2}^{n}(\mathbb{R})$ на $H_{+}^{2}\left(\mathbb{C}^{n}\right), m$ - некоторая положительная константа. Действительно, вводя обозначение $\Psi(z)=\left(e^{-i a z_{0}}-e^{-i a z}\right)^{-1}$, равенство (3.10) перепишем в виде

$$
z^{-1} \Psi(z)\left(z-z_{0}\right) K_{0}(z, h)=e^{-i a z} \Psi(z) e^{i a z} X(z, h)-\frac{z_{0}}{z} \Psi(z) X\left(z_{0}, h\right)+F(z, h) .
$$

Если считать, что $\operatorname{Im} z_{0}<-\varepsilon$, то в области $\operatorname{Im} z>-\varepsilon$ функции $\Psi(z), e^{-i a z} \Psi(z)$ ограничены и аналитичны. Рассматривая последнее равенство на прямой $\mathbb{R}-i \varepsilon$, получим

$$
\begin{aligned}
(x-i \varepsilon)^{-1} & \Psi(x-i \varepsilon)\left(x-i \varepsilon-z_{0}\right) v_{\varepsilon}(x+i 0) K_{0}(x-i \varepsilon, h) \\
=- & \frac{z_{0}}{x-i \varepsilon} \Psi(x-i \varepsilon) v_{\varepsilon}(x+i 0) X\left(z_{0}, h\right) \\
& \quad+e^{-i a x} \Psi(x-i \varepsilon) e^{i a x} v_{\varepsilon}(x+i 0) X(x-i \varepsilon, h)+\mathbb{P}_{+} v_{\varepsilon}(x+i 0) F(x-i \varepsilon, h) \\
& \quad+\mathbb{P}_{-} v_{\varepsilon}(x+i 0) F(x-i \varepsilon, h) .
\end{aligned}
$$

Из леммы 3.1 вытекает, что все слагаемые в правой части (3.13), кроме последнего, принадлежат пространству Харди $H_{+}^{2}\left(\mathbb{C}^{n}\right)$, что и доказывает неравенство (3.12).

Шаг 3. Предположим теперь, что оценка снизу (3.11) не имеет места, т. е. существует последовательность $h_{n} \in \mathcal{K}_{a}\left(\left\|h_{n}\right\|=1\right)$ такая, что

$$
\int_{\mathbb{R}}\left\|v_{\varepsilon}(x+i 0) K_{0}\left(x-i \varepsilon, h_{n}\right)\right\|^{2} d x \rightarrow 0, \quad n \rightarrow \infty .
$$

Тогда из (3.12) вытекает, что

$$
\mathbb{P}_{-} v_{\varepsilon}(x+i 0) F\left(x-i \varepsilon, h_{n}\right) \rightarrow 0, \quad n \rightarrow \infty,
$$

в метрике пространства $L_{2}^{n}(\mathbb{R})$. Докажем теперь, что

$$
\mathbb{P}_{+} v_{\varepsilon}(x+i 0) F\left(x-i \varepsilon, h_{n}\right) \rightarrow 0, \quad n \rightarrow \infty .
$$

Для этого сначала заметим, что

$$
w_{-}(x-i \varepsilon) F(x-i \varepsilon, h) \in H_{-}^{2}\left(\mathbb{C}^{n}\right), \quad h \in \mathcal{K}_{a} .
$$

Действительно, из формулы (2.3) вытекает представление

$$
w_{-}(x-i \varepsilon) F(x-i \varepsilon, h)=w_{-}(x-i \varepsilon) \Phi_{a}^{-1}(x-i \varepsilon) \varphi(x-i \varepsilon, h),
$$

где $\varphi(z, h):=\operatorname{col}\left(\left(I-z B^{*}\right)^{-1} h, f_{k}^{a}\right)_{k=1}^{n}$. Таким образом,

$$
{ }^{t}\left(w_{-}(x-i \varepsilon) F(x-i \varepsilon)\right)={ }^{t} \varphi(x-i \varepsilon, h)\left({ }^{t} W_{-}(x)\right)^{-1}, \quad h \in \mathcal{K}_{a},
$$

причем в силу условий леммы вес $W_{-}(x):=\Phi_{a}(x-i \varepsilon) w_{-}^{-1}(x-i \varepsilon)$ участвует в факторизации вида

$$
\left({ }^{t} W_{-}(x)\right)^{*}\left({ }^{t} W_{-}(x)\right)={ }^{t}\left(W_{-}(x) W_{-}^{*}(x)\right)={ }^{t} W_{\varepsilon}^{2}(x) \in \mathcal{M}_{n}^{2} .
$$


Из теоремы 2.1' вытекает оценка

$$
\int_{\mathbb{R}}\left\|^{t} \varphi(x-i \varepsilon, h)\left({ }^{t} W_{-}(x)\right)^{-1}\right\|^{2} d x=\int_{\mathbb{R}-i \varepsilon}\left\|w_{-}(z) F(z, h)\right\|^{2} d z \leqslant M\|h\|^{2} .
$$

Следовательно, для строки ${ }^{t} \varphi(x-i \varepsilon, h)$ применима теорема 1.3 , в силу которой эта строка находится в образе преобразования $\mathcal{B}_{a}(z, h)$. Теперь из теоремы 1.2 вытекает включение

$$
{ }^{t} \varphi(x-i \varepsilon, h)\left({ }^{t} W_{-}(x)\right)^{-1} \in{ }^{*} H_{-}^{2}\left(\mathbb{C}^{n}\right), \quad h \in \mathcal{K}_{a},
$$

что, как это следует из (3.17), и требовалось доказать.

Если рассмотреть (3.16) на введенной ранее последовательности $h_{n}$, то мы получим

$$
\begin{aligned}
0= & \mathbb{P}_{+} w_{-}(x-i \varepsilon) F\left(x-i \varepsilon, h_{n}\right)=\mathbb{P}_{+} w_{-}(x-i \varepsilon) v_{\varepsilon}^{-1}(x+i 0) v_{\varepsilon}(x+i 0) F\left(x-i \varepsilon, h_{n}\right) \\
=\mathbb{P}_{+} w_{-}(x-i \varepsilon) v_{\varepsilon}^{-1}(x & +i 0) \mathbb{P}_{+} v_{\varepsilon}(x+i 0) F\left(x-i \varepsilon, h_{n}\right) \\
& \quad+\mathbb{P}_{+} w_{-}(x-i \varepsilon) v_{\varepsilon}^{-1}(x+i 0) \mathbb{P}_{-} v_{\varepsilon}(x+i 0) F\left(x-i \varepsilon, h_{n}\right) .
\end{aligned}
$$

Из $\left(3.14^{\prime}\right)$ следует, что второе слагаемое в последнем равенстве стремится к нулю. Поэтому в силу леммы 2.2 об операторе Теплица из стремления к нулю первого слагаемого следует справедливость (3.15).

Рассмотрим теперь равенство (3.13) для последовательности $h_{n}$, которую можно считать слабо сходящейся к некоторому вектору $h_{0} \in \mathcal{K}_{a}$. Если учесть (3.14), (3.14') $(3.15)$, а также оценку снизу $|\Psi(x-i \varepsilon)| \geqslant \delta>0, x \in \mathbb{R}$, то из (3.13) заключаем, что

$$
v_{\varepsilon}(x+i 0) X\left(x-i \varepsilon, h_{n}\right)-\frac{z_{0} v_{\varepsilon}(x+i 0)}{x-i \varepsilon} X\left(z_{0}, h_{n}\right) \rightarrow 0, \quad n \rightarrow \infty,
$$

в метрике пространства $L_{2}^{n}(\mathbb{R})$. Из определения функционала $X(z, h)$ вытекает сходимость $X\left(z_{0}, h_{n}\right) \rightarrow X\left(z_{0}, h_{0}\right)$ в $\mathbb{C}^{n}$. Теперь из $(3.18)$ следует сходимость

$$
X\left(x-i \varepsilon, h_{n}\right) \rightarrow \frac{z_{0} X\left(z_{0}, h_{0}\right)}{x-i \varepsilon}, \quad n \rightarrow \infty,
$$

в $L_{2}^{n}\left(v_{\varepsilon}^{2}\right)$, где вес $v_{\varepsilon}^{2}(x):=v_{\varepsilon}^{*}(x+i 0) v_{\varepsilon}(x+i 0)$ принадлежит классу $\mathcal{M}_{n}^{2}$.

В силу аналога теоремы 1.3 и аналога следствия 1.1 для целых столбцов следует, что предел последовательности $X\left(x-i \varepsilon, h_{n}\right)$ в метрике $L_{2}\left(v_{\varepsilon}^{2}\right)$ должен быть целой вектор-функцией. Поэтому из (3.19) выводим, что $X\left(z_{0}, h_{0}\right)=0$, стало быть, в силу (3.18) имеем

$$
\int_{\mathbb{R}}\left\|v_{\varepsilon}(x+i 0) X\left(x-i \varepsilon, h_{n}\right)\right\|^{2} d x \rightarrow 0, \quad n \rightarrow \infty .
$$

Учитывая, что $w_{-}^{*}(x-i \varepsilon) w_{-}(x-i \varepsilon) \stackrel{\text { п.в. }}{=} v_{\varepsilon}^{*}(x+i 0) v_{\varepsilon}(x+i 0)$, из (3.6) получаем равенство $\lim _{n \rightarrow \infty} h_{n}=0$, что противоречит предположению $\left\|h_{n}\right\|=1$. Лемма доказана.

3.2. В этом пункте рассмотрим модельную задачу о принадлежности полугруппы $U_{+}(t)$ классу $C_{0}$. Напомним, что оператор $K_{a}$, построенный по $\Theta(z)=$ $e^{i a z} E_{n}$ и весу $w^{2} \in \mathcal{M}_{n}^{2}$, задается формулой (3.1), матрица-функция $\Phi_{a}(z)$ определяется формулой (3.2). 
Лемма 3.3. Пусть оператор $K_{a}$ при некотором $\varepsilon>0$ удовлетворяет условиям:

1) вес $W_{\varepsilon}^{2}(x):=\Phi_{a}(x-i \varepsilon)\left(w_{-}^{*}(x-i \varepsilon) w_{-}(x-i \varepsilon)\right)^{-1} \Phi_{a}^{*}(x-i \varepsilon), x \in \mathbb{R}$, принадлежит классу $\mathcal{M}_{n}^{2}$;

2) матрица-функиия $W_{-}(z):=\Phi_{a}(z-i \varepsilon) w_{-}^{-1}(z-i \varepsilon)$ внешняя в области $\mathbb{C}_{-}$.

Тогда $\operatorname{Ker} K_{a}=\operatorname{Ker} K_{a}^{*}=\{0\}$ и оператор $A:=K_{a}^{-1}$ генерирует полугрупnу $U_{+}(t)=\exp \{i A t\}, t \geqslant 0$, класса $C_{0}$, причем ее экспоненииальныи тип не превосходит $\varepsilon$.

ДокАзАтЕльство. Шаг 1. Докажем сначала двустороннюю оценку

$$
m\|h\|^{2} \leqslant \int_{\mathbb{R}}\left\|\mathbb{P}_{-} v_{\varepsilon}(x+i 0) K_{0}(x-i \varepsilon, h)\right\|^{2} d x \leqslant M\|h\|^{2}, \quad h \in \mathcal{K}_{a},
$$

где функционал $K_{0}(z, h)$ определяется формулой $(3.9), m, M$ - положительные константы. Из леммы 3.2 следует, что достаточно доказать лишь оценку снизу. Прежде всего заметим, что

$$
\mathbb{P}_{+} w_{-}(x-i \varepsilon) K_{0}(x-i \varepsilon, h)=0, \quad h \in \mathcal{K}_{a} .
$$

Чтобы убедиться в этом, умножим слева обе части равенства $(3.10)$ на $w(x-i \varepsilon)$, в котором предварительно положим $z=x-i \varepsilon$. Тогда первое слагаемое в правой части преобразованного равенства принадлежит $H_{-}^{2}\left(\mathbb{C}^{n}\right)$ в силу утверждения 2) леммы 3.1 , принадлежность классу $H_{-}^{2}\left(\mathbb{C}^{n}\right)$ второго слагаемого вытекает из (3.16).

Введем теперь оператор

$$
S h:=\mathbb{P}_{-} v_{\varepsilon}(x+i 0) K_{0}(x-i \varepsilon, h), \quad h \in \mathcal{K}_{a},
$$

и предположим, что оценка снизу (3.20) места не имеет, т. е. для некоторой последовательности $h_{n}\left(\left\|h_{n}\right\|=1\right)$ последовательность $S h_{n}$ стремится к нулю при $n \rightarrow \infty$. Тогда из равенства (3.21) следует, что

$$
\begin{aligned}
& 0= \mathbb{P}_{+} w_{-}(x-i \varepsilon) K_{0}\left(x-i \varepsilon, h_{n}\right) \\
&=\mathbb{P}_{+} w_{-}(x-i \varepsilon) v_{\varepsilon}^{-1}(x+i 0) \mathbb{P}_{+} v_{\varepsilon}(x+i 0) K_{0}\left(x-i \varepsilon, h_{n}\right) \\
&+\mathbb{P}_{+} w_{-}(x-i \varepsilon) v_{\varepsilon}^{-1}(x+i 0) S h_{n},
\end{aligned}
$$

и поэтому стремится к нулю первое слагаемое справа. Из леммы 2.2 вытекает стремление к нулю последовательности $\mathbb{P}_{+} v_{\varepsilon}(x+i 0) K_{0}\left(x-i \varepsilon, h_{n}\right)$, что вместе с $S h_{n} \rightarrow 0, n \rightarrow \infty$, влечет равенство $\lim _{n \rightarrow \infty} v_{\varepsilon}(x+i 0) K_{0}\left(x-i \varepsilon, h_{n}\right)=0$ в метрике $L_{2}^{n}(\mathbb{R})$. Однако тогда из леммы 3.2 следует, что $h_{n} \rightarrow 0, n \rightarrow \infty$. Полученное противоречие доказывает оценку снизу (3.20).

Шаг 2. Таким образом, оператор $S$ - изоморфизм пространства $\mathcal{K}_{a}$ на некоторое подпространство $\mathcal{L}$ пространства Харди $H_{-}^{2}\left(\mathbb{C}^{n}\right)$. Вычисления, аналогичные тем, которые проводились при выводе (2.17), показывают, что справедливо равенство

$$
S K_{a}\left(I-(\mu-i \varepsilon) K_{a}\right)^{-1} h=\frac{(S h)(x)-(S h)(\mu)}{x-\mu}, \quad \operatorname{Im} \mu<0,
$$

для всех $h \in \mathcal{K}_{a}$. Заметим, что $(\mu-i \varepsilon)^{-1} \notin \sigma\left(K_{a}\right), \operatorname{Im} \mu<0$, поскольку по условию функция $W_{-}(z)$ является внешней в $\mathbb{C}_{-}$. Таким образом, подпространство $\mathcal{L}$ инвариантно относительно действия операторов

$$
\left(R_{\mu} \varphi\right)(x)=\frac{\varphi(x)-\varphi(\mu)}{x-\mu}, \quad \varphi \in \mathcal{L}, \quad \mu \in \mathbb{C}_{-} .
$$


Поэтому существует [13], [24] мероморфная в $\mathbb{C}$ и внутренняя в $\mathbb{C}_{-}$матрицафункция П $(z)$ такая, что

$$
\mathcal{L}=H_{-}^{2}\left(\mathbb{C}^{n}\right) \ominus \Pi(z) H_{-}^{2}\left(\mathbb{C}^{n}\right) .
$$

Из (3.22) вытекает, что вектор-функции из $\mathcal{L}$ аналитичны в окрестности нуля. Полагая $\mu=0$ в (3.22), получаем равенство

$$
S K_{a}\left(I+i \varepsilon K_{a}\right)^{-1} h=L S h, \quad h \in \mathcal{K}_{a},
$$

где антидиссипативный оператор $L$ действует по формуле

$$
(L \varphi)(z)=z^{-1}(\varphi(z)-\varphi(0)), \quad \varphi \in \mathcal{L} .
$$

Отсюда легко вытекает, что $\operatorname{Ker} K_{a}=\operatorname{Ker} K_{a}^{*}=\{0\}$ и оператор $A:=K_{a}^{-1}$ есть $w$-возмущение оператора $A_{0}:=\left(B^{*}\right)^{-1}$. Таким образом, последнее равенство переписывается в виде

$$
(A+i \varepsilon I)^{-1}=S^{-1} L S .
$$

Поэтому полугруппа $U_{+}(t)$ принадлежит классу $C_{0}$ и имеет вид $U_{+}(t)=$ $e^{\varepsilon t} S^{-1} \exp \left\{i L^{-1} t\right\} S$, причем полугруппа $\exp \left\{i L^{-1} t\right\}$ является сжимающей. Лемма доказана.

Перейдем теперь к рассмотрению общего модельного оператора $K$, который порождается парой $\Theta \in J_{n}$ и $w^{2} \in \mathcal{M}_{n}^{2}$ и действует по формуле

$$
K h=B^{*} h+\sum_{k=1}^{n}\left(h, f_{k}^{\Theta}\right) g_{k}^{\Theta}, \quad h \in \mathcal{K}_{\Theta} .
$$

Напомним, что $\Delta(z)=\operatorname{det} \Phi(z)$, где матрица-функция $\Phi(z)$ по оператору $K$ задается формулами (2.3). Через $\Lambda$ обозначается множество корней функции $\Delta$.

Мы предполагаем, что $\operatorname{Ker} K_{a}=\operatorname{Ker} K_{a}^{*}=\{0\}$, и рассматриваем оператор $A:=K^{-1}$, который является $w$-возмущением оператора $A_{0}:=\left(B^{*}\right)^{-1}$ ранга $n$.

Теорема 3.1. Пусть оператор $K$ порождается матрищей-функкией $\Theta \in J_{n} u$ весом $w^{2} \in \mathcal{M}_{n}^{2}$. Пусть при некотором $\varepsilon \geqslant 0$ выполняются условия:

1) $\inf _{\lambda_{k} \in \Lambda} \operatorname{Im} \lambda_{k}>-\varepsilon$;

2) $\lim \sup _{y \rightarrow-\infty}|y|^{-1} \log |\Delta(i y)|=0$;

3) вес $W_{\varepsilon}^{2}(x):=\Phi(x-i \varepsilon)\left(w_{-}^{*}(x-i \varepsilon) w_{-}(x-i \varepsilon)\right)^{-1} \Phi^{*}(x-i \varepsilon)$ удовлетворяет условию $\left(A_{2}\right)$.

Тогда полугруппа $U_{+}(t)=\exp \{i A t\}$ принадлежст классу $C_{0}$ и ее экспоненицальный тип не превосходит $\varepsilon$.

ДоказАтельство. Обозначим через а экспоненциальный тип матрицыфункции $\Theta$. Наряду с оператором $K$, который задается формулой (3.23) в пространстве $\mathcal{K}_{\Theta}$, рассмотрим также оператор $K_{a}$ вида $(3.1)$ в пространстве $\mathcal{K}_{a}$ :

$$
K_{a} h=B_{a}^{*} h+\sum_{k=1}^{n}\left(h, f_{k}^{\Theta}\right) g_{k}^{a}, \quad h \in \mathcal{K}_{a} .
$$

Заметим, что из леммы 1.2 вытекают равенства

$$
\mathbb{P}_{\Theta}\left(I-z B_{a}^{*}\right)^{-1} g_{k}^{a}=\left(I-z B^{*}\right)^{-1} g_{k}^{\Theta}, \quad 1 \leqslant k \leqslant n .
$$


Поэтому, вычисляя по формуле (3.2) матрицу-функцию $\Phi_{a}(z)$, получим

$$
\begin{aligned}
\Phi_{a}^{k j}(z) & =\delta_{k j}-z\left(\left(I-z B_{a}^{*}\right)^{-1} g_{j}^{a}, f_{k}^{\Theta}\right)=\delta_{k j}-z\left(\mathbb{P}_{\Theta}\left(I-z B_{a}^{*}\right)^{-1} g_{j}^{a}, f_{k}^{\Theta}\right) \\
& =\delta_{k j}-z\left(\left(I-z B^{*}\right)^{-1} g_{j}^{\Theta}, f_{k}^{\Theta}\right)=\Phi_{k j}(z), \quad 1 \leqslant k, j \leqslant n,
\end{aligned}
$$

т. е. $\Phi_{a}(z) \equiv \Phi(z)$. Заметим, что из условий 1$\left.), 2\right)$ и теоремы 1.2 вытекает тот факт, что матрица-функция $W_{-}(z)=\Phi_{a}(z-i \varepsilon) w_{-}^{-1}(z-i \varepsilon)$ является внешней в $\mathbb{C}_{-}$. В самом деле, из теоремы 1.2 следует, что элементы матрицы $(z-i \varepsilon)^{-1} \Phi_{a}(z-i \varepsilon) w_{-}(z-i \varepsilon)$ принадлежат классу $H_{-}^{2}$. Поэтому имеет место факторизация

$$
\Phi_{a}(z-i \varepsilon) w_{-}(z-i \varepsilon)=W_{e}(z) V(z),
$$

где $W_{e}$ - внешняя, $V$ - внутренняя матрицы-функции в $\mathbb{C}_{-}$. Переходя к определителям, из условия 2) выводим, что $V$ - унитарная константа. Таким образом, оператор $K_{a}$ удовлетворяет всем условиям леммы 3.3 , следовательно, полугруппа $\exp \left\{i K_{a}^{-1} t\right\}, t \geqslant 0$, принадлежит классу $C_{0}$.

Далее, поскольку имеет место факторизация $e^{i a z} E_{n}=\Theta(z) \Theta_{1}(z), \Theta, \Theta_{1} \in J_{n}$, то [17] пространство $\mathcal{K}_{\Theta}$ инвариантно относительно оператора $B_{a}$ и

$$
B=B_{a}\left|\mathcal{K}_{\Theta}, \quad B^{*}=\mathbb{P}_{\Theta} B_{a}^{*}\right| \mathcal{K}_{\Theta} .
$$

Поэтому из определения оператора $K_{a}$ и формулы (3.23) вытекает равенство

$$
\mathbb{P}_{\Theta} K_{a} h=K h, \quad h \in \mathcal{K}_{\Theta} .
$$

Из приведенных формул следует, что

$$
(A-z I)^{-1}=\mathbb{P}_{\Theta}\left(K_{a}^{-1}-z I\right)^{-1} \mid \mathcal{K}_{\Theta}, \quad z \notin \sigma(A) .
$$

Таким образом, полугруппа $U_{+}(t)=\exp \{i A t\}$ также принадлежит классу $C_{0}$, и ее тип не превосходит типа полугруппы $\exp \left\{i K_{a}^{-1} t\right\}$. Теорема доказана.

Докажем теперь необходимость условий 1)-3) теоремы 3.1.

Лемма 3.4. Пусть оператор $K$ задается формулой (3.23) и $\operatorname{Ker} K=$ Ker $K^{*}=\{0\}$. Пусть nолугруппа $U_{+}(t)=\exp \{i A t\}$, где $A:=K^{-1}$, принадлежит классу $C_{0}$ и ее экспоненциальный тип равен $\omega$. Тогда для любого $\varepsilon>\max \{0, \omega\}$ выполняются условия 1), 2) теоремы 3.1. Если матрица-функиия $\Theta(z)$ такова, что при некотором $\delta>0$ функиия $e^{-i \delta z} \Theta(z)$ ограничена в $\mathbb{C}_{+}$, то при каждом $\varepsilon>\max \{0, \omega\}$ выполняется условие 3) теоремы 3.1 .

ДокАзАтельство. Необходимость условия 1) теоремы 3.1 очевидна. Доказательство необходимости условия 3) при дополнительном ограничении, налагаемом на $\Theta(z)$, аналогично доказательству для случая полугруппы $U_{-}(t)$ и также основано на лемме 2.4 .

Перейдем теперь к доказательству необходимости условия 2). Пусть полугруппа $U_{+}(t)$ принадлежит классу $C_{0}$. Тогда в области $\operatorname{Im} z<-\varepsilon, \varepsilon>$ $\min \{0, \omega\}$, имеет место представление

$$
i(A-z I)^{-1}=\int_{0}^{\infty} U_{+}(t) e^{-i z t} d t .
$$

Повторяя рассуждения из доказательства леммы 2.3 (шаг 2), заключаем, что матрица-функция $\Phi^{-1}(z)\left(z-z_{0}\right)^{-2}, \operatorname{Im} z_{0}>0$, ограничена в области $\operatorname{Im} z<-\varepsilon$. 
Из определения $\Phi(z)$ (формула $(2.3))$ и того факта, что $A_{0}:=\left(B^{*}\right)^{-1}$ является диссипативным оператором, следует ограниченность матрицы-функции $\Phi(z)\left(z-z_{0}\right)^{-2}$ в полуплоскости $\operatorname{Im} z<-\varepsilon$. Поэтому функция $\Delta(z)\left(z-z_{0}\right)^{-2 n}$ ограничена в области $\operatorname{Im} z<-\varepsilon$. Отсюда вытекает, что $\Delta(z-i \varepsilon)-$ функция ограниченного вида в $\mathbb{C}_{-}[8]$, и поэтому она факторизуется следующим образом:

$$
\Delta(z-i \varepsilon)=e^{-i \alpha z} \varphi(z), \quad \alpha \geqslant 0,
$$

где $\varphi$ - внешняя функция в $\mathbb{C}_{-}$.

Обозначим через $\Phi^{0}(z)$ матрицу, присоединенную к $\Phi(z)$. Поскольку матрица-функция $\Phi(z)\left(z-z_{0}\right)^{-2}$ ограничена в области $\operatorname{Im} z<-\varepsilon$, то элементы $\Phi_{k j}^{0}(z-i \varepsilon)$ допускают следующие факторизации в $\mathbb{C}_{-}$:

$$
\Phi_{k j}^{0}(z-i \varepsilon)=e^{-i p_{k j} z} \varphi_{k j}(z), \quad p_{k j} \geqslant 0,
$$

$\varphi_{k j}-$ внешние функции в $\mathbb{C}_{-}$. Из $(3.24)$ следует, что

$$
\begin{aligned}
\left\{\Phi^{-1}(z-i \varepsilon)\left(z-i \varepsilon-z_{0}\right)^{-2}\right\}_{k j} & =\Phi_{k j}^{0}(z-i \varepsilon) \Delta^{-1}(z-i \varepsilon)\left(z-i \varepsilon-z_{0}\right)^{-2} \\
& =e^{-i\left(p_{k j}-\alpha\right) z} \Psi_{k j}(z),
\end{aligned}
$$

где $\Psi_{k j}-$ внешние функции в $\mathbb{C}_{-}$. Ввиду ограниченности $\Phi^{-1}(z)\left(z-z_{0}\right)^{-2}$ отсюда вытекают равенства

$$
p_{k j}=\alpha+\varepsilon_{k j}, \quad \varepsilon_{k j} \geqslant 0 .
$$

Поэтому из (3.25) следует представление

$$
\operatorname{det} \Phi^{0}(z-i \varepsilon)=e^{-i n \alpha z} \Psi(z), \quad z \in \mathbb{C}_{-},
$$

причем функция $\Psi$ после деления на $(z-i)^{2(n+1)}$ будет ограниченной в $\mathbb{C}_{-}$. С другой стороны, из (3.24) следует формула

$$
\operatorname{det} \Phi^{0}(z-i \varepsilon)=\Delta^{n-1}(z-i \varepsilon)=e^{-i(n-1) \alpha z} \varphi^{n-1}(z),
$$

что можно совместить с предыдущим равенством лишь в случае $\alpha=0$. Тогда из (3.24) следует, что функция $\Delta(z-i \varepsilon)$ является внешней, т. е. условие 2$)$ теоремы 3.1 выполняется. Лемма доказана.

3.3. В этом пункте мы опишем сильно непрерывные полугруппы $U_{+}(t)=$ $\exp \{i A t\}$, где оператор $A$ принадлежит классу $w$-возмущений конечного ранга. Напомним, в п. 1.5 было показано, что каждое $w$-возмущение $A$ оператора $A_{0} \in \Sigma^{(\exp )}$ ранга $n$ каноническим образом порождает вес $w^{2} \in \mathcal{M}_{n}^{2}$ и матрицу-функцию $\Theta \in J_{n}$. Кроме того, каждому оператору $A$ в силу формул (2.27) отвечает целая матрица-функция $\Phi$ такая, что множество $\Lambda$ корней функции $\Delta(z)=\operatorname{det} \Phi(z)$ совпадает со спектром оператора $A$. Имеет место

Tеорема 3.2. Пусть оператор $A$ есть w-возмущение оператора $A_{0} \in$ $\Sigma^{(\exp )}$, которому отвечает пара $w^{2} \in \mathcal{M}_{n}^{2}, \Theta \in J_{n}$. Пусть при некотором $\varepsilon \geqslant 0$ выполняются условия:

1) $\inf _{\lambda_{k} \in \Lambda} \operatorname{Im} \lambda_{k}>-\varepsilon$

2) $\lim \sup _{y \rightarrow-\infty}|y|^{-1} \log |\Delta(i y)|=0$;

3) матричныци вес $W_{\varepsilon}^{2}(x):=\Phi(x-i \varepsilon)\left(w_{-}^{*}(x-i \varepsilon) w_{-}(x-i \varepsilon)\right)^{-1} \Phi^{*}(x-i \varepsilon)$ удовлетворяет условию $\left(A_{2}\right)$ на $\mathbb{R}$. 
Тогда полугруппа $U_{+}(t):=\exp \{i A t\}, t \geqslant 0$, принадлежит классу $C_{0}$ u ее экспоненииальный тип не превосходит $\varepsilon$.

Обратно, если $U_{+}(t) \in C_{0}$ и ее тип равен $\omega, \operatorname{mo~при~любом~} \varepsilon>\max \{0, \omega\}$ выполняются условия 1), 2). Если при некотором $\delta>0$ матрица-функиия $e^{-i \delta z} \Theta(z)$ ограничена в $\mathbb{C}_{+}$, то при любом $\varepsilon>\max \{0, \omega\}$ выполняется условие 3).

ДокАЗАТЕЛьСтво. С помощью теоремы 1.5 задача сводится к случаю модельного оператора $K$, который задается формулой (3.23). Дальнейшие рассуждения полностью повторяют доказательство теоремы 2.3 и опираются на теорему 3.1 и лемму 3.4. Теорема доказана.

СледСтвиЕ 3.1. Пусть $A-w$-возмущение оператора $A_{0} \in \Sigma^{(\exp )}$. Ecли при некотором $\varepsilon>0$ выполняются условия 1)-3) теоремы 3.2, то оператор $\left(A^{*}-i \varepsilon I\right)^{-1}$ подобен некоторому диссипативному оператору с не более чем $n$-мерной мнимой частью.

ДокАЗАТЕЛЬСтво. Из теоремы 1.5 следует, что это утверждение достаточно доказать для модельного оператора $K$ из теоремы 3.1. Как и при доказательстве теоремы 3.1, наряду с оператором

$$
K h=B^{*} h+\sum_{k=1}^{n}\left(h, f_{k}^{\Theta}\right) g_{k}^{\Theta}, \quad h \in \mathcal{K}_{\Theta}
$$

рассмотрим также оператор

$$
K_{a} h=B_{a}^{*} h+\sum_{k=1}^{n}\left(h, f_{k}^{\Theta}\right) g_{k}^{a}, \quad h \in \mathcal{K}_{a} .
$$

Поскольку $B_{a} \mid \mathcal{K}_{\Theta}=B$ и $g_{k}^{\Theta}=\mathbb{P}_{\Theta} g_{k}^{a}, 1 \leqslant k \leqslant n$, при любом $h \in \mathcal{K}_{\Theta}$ получим

$$
K_{a}^{*} h=B_{a} h+\sum_{k=1}^{n}\left(h, g_{k}^{a}\right) f_{k}^{\Theta}=B h+\sum_{k=1}^{n}\left(h, g_{k}^{\Theta}\right) f_{k}^{\Theta}=K^{*} h .
$$

Следовательно, справедливо равенство

$$
\left(\left(K^{*}\right)^{-1}-i \varepsilon I\right)^{-1}=\left(\left(K_{a}^{*}\right)^{-1}-i \varepsilon I\right)^{-1} \mid \mathcal{K}_{\Theta} .
$$

При доказательстве леммы 3.3 было установлено, что оператор $\left(\left(K_{a}\right)^{-1}+i \varepsilon I\right)^{-1}$ подобен некоторому антидиссипативному оператору с не более чем $n$-мерной мнимой частью. Поэтому оператор $\left(A^{*}-i \varepsilon I\right)^{-1}$, где $A=K^{-1}$, подобен некоторому диссипативному оператору $L$. Лемма доказана.

3.4. В этом пункте мы сделаем ряд дополнений к доказанным теоремам 2.3, 3.2. Начнем со следующего простого наблюдения. Пусть замкнутый плотно заданный оператор $A$ в абстрактном гильбертовом пространстве $\mathfrak{H}$ задается формулой

$$
A^{-1} h=B^{*} h+\sum_{k=1}^{n}\left(h, f_{k}\right) g_{k},
$$

где $B$ - вольтерров диссипативный оператор с тривиальным ядром и $n$-мерной мнимой частью $\operatorname{Im} B$. 
Теорема 3.3. Пусть в гилъбертовом пространстве $\mathfrak{H}$ оператор $A$ задается формулой (3.26), в которой векторь $\left\{g_{k}\right\}_{k=1}^{n}$ образуют базис подпространства несамосопряженности $\mathcal{L}:=(\operatorname{Im} B) \mathfrak{H}$. Тогда оператор $A$ является $w$-возмущением $A_{0}=\left(B^{*}\right)^{-1}$, которому отвечает вес Макенхаупта $w^{2}(x) \equiv E_{n}, x \in \mathbb{R}$.

ДокАзАтельство. Оператор $B^{*}$ унитарно эквивалентен своей функциональной модели вида (1.4), причем при такой унитарной эквивалентности базис подпространства $\mathcal{L}$ переходит в базис подпространства несамосопряженности оператора (1.4) [17]. Поэтому можно считать, что в (3.26) оператор $B^{*}$ действует по формуле (1.4) в пространстве $\mathcal{K}_{\Theta}$. В замечании 2.1 доказано, что образ оператора $B-B^{*}$ натягивается на векторы $z^{-1}\left(E_{n}-\Theta(z)\right) e_{k}, 1 \leqslant k \leqslant n$, где $e_{k}$ - стандартные орты в $\mathbb{C}^{n}$. В замечании 2.1 также была проверена справедливость равенств

$$
\left(I+i B^{*}\right) \mathbb{P}_{\Theta} \frac{e_{k}}{x+i}=z^{-1}\left(E_{n}-\Theta(z)\right) e_{k}, \quad k=1,2, \ldots, n .
$$

Таким образом, системы векторов

$$
g_{k}:=\left(I+i B^{*}\right) \mathbb{P}_{\Theta} \frac{Q e_{k}}{x+i}, \quad 1 \leqslant k \leqslant n
$$

где $Q$ - произвольная обратимая матрица, описывают все базисы подпространства $\mathcal{L}$ для модельного оператора. С другой стороны, введенные векторы $g_{k}$ удовлетворяют соотношениям (1.6) в случае веса $w^{2}(x)=Q^{*} Q$. Из теоремы 1.1 следует, что $A-w$-возмущение оператора $A_{0}=\left(B^{*}\right)^{-1}$, которому отвечает вес $Q^{*} Q$, а значит, и вес $E_{n}$. Теорема доказана.

Во введении было отмечено, что в теореме о полугруппе $U_{-}(t)=\exp \{-i A t\}$, вообще говоря, нельзя избавиться от условия того, что оператор $A$ является $w$-возмущением $A_{0} \in \Sigma^{(\exp )}$ с некоторым весом Макенхаупта $w^{2}$. Здесь мы выделим класс операторов вида (3.26) таких, что условие: оператор $A$ есть $w$-возмущение оператора $A_{0}=\left(B^{*}\right)^{-1}$, является необходимым для принадлежности $\exp \{-i A t\}$ классу $C_{0}$. Сформулируем точный результат. Как и раньше, будем считать, что в формуле (3.26) оператор $B$ является диссипативным с $n$-мерной мнимой частью и со спектром в нуле. Априори на системы векторов $\left\{f_{k}\right\},\left\{g_{k}\right\}$ ограничения, кроме их линейной независимости, не налагаются. В следующей формулировке через $\Theta(z)$ обозначается характеристическая матрица-функция оператора $B$.

Теорема 3.4. Пусть А действует по формуле (3.26) в сепарабельном гильбертовом пространстве $\mathfrak{H}$. Пусть также векторы $f_{k}, 1 \leqslant k \leqslant n$, образуют базис подпространства несамосопряженности $(\operatorname{Im} B) \mathfrak{H}$ и существует такое $\delta>0$, что элементы матрицы $e^{-i \delta z} \Theta(z)$ ограничены в $\mathbb{C}_{+}$. Тогда если $A$ генеpupyem полугруппу $\exp \{-i A t\}, t \geqslant 0$, класса $C_{0}$, то существует вес $w^{2} \in \mathcal{M}_{n}^{2}$ такой, что $A$ есть w-возмущение оператора $A_{0}=\left(B^{*}\right)^{-1}$ класса $\Sigma^{(\exp )}$.

ДокАЗАТЕЛЬство. Шаг 1. Пусть полугруппа $\exp \{-i A t\}$ принадлежит классу $C_{0}$. Тогда в силу резольвентного критерия принадлежности полугруппы классу $C_{0}[2]$ следует, что оператор $-A^{*}$ также генерирует $C_{0}$-полугруппу $\exp \left\{-i A^{*} t\right\}$. 
Заметим, что

$$
\left(-A^{*}\right)^{-1} h=B_{1}^{*} h+\sum_{k=1}^{n}\left(h, g_{k}\right)\left(-f_{k}\right), \quad h \in \mathfrak{H},
$$

где введено обозначение $B_{1}^{*}:=-B$. Оператор $B_{1}$ принадлежит тому же классу, что и оператор $B$, и между их характеристическими матрицами-функциями $\Theta$ и $\Theta_{1}$ (см. формулы $\left.(1.5),\left(1.5^{\prime}\right)\right)$ существует простая связь:

$$
\Theta_{1}(z)=\Theta^{*}(-\bar{z}), \quad z \in \mathbb{C} .
$$

Поскольку для операторов $B$ и $B_{1}$ подпространства несамосопряженности совпадают, из (3.27) и теоремы 3.3 вытекает, что оператор $-A^{*}$ есть $w$-возмущение $\left(B_{1}^{*}\right)^{-1}$, которому отвечает вес $w^{2}(x) \equiv E_{n}$. Как уже отмечалось, оператор $\left(-A^{*}\right)^{-1}$ унитарно эквивалентен оператору вида $(2.1)$, который порождается $\Theta_{1} \in J_{n}$ и весом Макенхаупта $E_{n}$. Поскольку существует $\delta>0$ такое, что функция $e^{-i \delta z} \Theta_{1}(z)$ ограничена в $\mathbb{C}_{+}$, то в силу леммы 2.5 при некотором $\varepsilon>0$ соответствующий вес $W_{\varepsilon}^{2}(x)$ принадлежит $\mathcal{M}_{n}^{2}$. В силу леммы 2.3 выполняется условие (2.20), которое равносильно тому, что матрица-функция

$$
W_{+}(z)=\Phi_{1}(z+i \varepsilon) \Theta_{1}(z+i \varepsilon), \quad z \in \mathbb{C}_{+},
$$

является внешней. Здесь через $\Phi_{1}(z)$ обозначена матрица-функция, которая вычисляется по формуле (2.11) для оператора вида (3.27). Поскольку в нашем случае $w_{+}(z) \equiv E_{n}$, в силу теоремы 2.1 и леммы 2.1 при указанном $\varepsilon$ имеет место двусторонняя оценка

$$
m\|h\|^{2} \leqslant \int_{\mathbb{R}+i \varepsilon}\|F(h, z)\|^{2} d z \leqslant M\|h\|^{2}, \quad h \in \mathcal{K}_{\Theta_{1}},
$$

где вектор-функция $F(h, z)$ вычисляется по формулам $(2.3),(2.4)$. Если снова рассмотреть оператор $-A^{*}$ в пространстве $\mathfrak{H}$, то с учетом $(3.27)$ получим

$$
F(h, z)=\Phi_{1}^{-1}(z) \operatorname{col}\left\{\left(\left(I-z B_{1}^{*}\right)^{-1} h, g_{k}\right)\right\}_{k=1}^{n}, \quad h \in \mathfrak{H} .
$$

Учитывая равенство $B_{1}^{*}=-B$, вычислим

$$
\begin{aligned}
\|F(h, z)\|^{2}=\left\|F^{*}(h, z)\right\|^{2} & =\left\|\operatorname{row}\left\{\left(\left(I-\bar{z} B_{1}\right)^{-1} g_{k}, h\right)\right\}_{k=1}^{n}\left(\Phi_{1}^{*}(z)\right)^{-1}\right\|^{2} \\
& =\left\|\operatorname{row}\left\{\left(\left(I+\bar{z} B^{*}\right)^{-1} g_{k}, h\right)\right\}_{k=1}^{n} V^{-1}(z)\right\|^{2}
\end{aligned}
$$

где введено обозначение $V^{2}(z):=\Phi_{1}(z) \Phi_{1}^{*}(z)$. Поскольку в нашем случае $w_{+}(z) \equiv E_{n}$, для веса $W_{\varepsilon}^{2}(x)$ справедлива формула

$$
W_{\varepsilon}^{2}(x)=\Phi_{1}(x+i \varepsilon) \Phi_{1}^{*}(x+i \varepsilon)=V^{2}(x+i \varepsilon) \in \mathcal{M}_{n}^{2} .
$$

Таким образом, из (3.28) вытекает справедливость двусторонней оценки

$$
m\|h\|^{2} \leqslant \int_{\mathbb{R}}\left\|\operatorname{row}\left\{\left(\left(I+(x-i \varepsilon) B^{*}\right)^{-1} g_{k}, h\right)\right\}_{k=1}^{n} V^{-1}(x+i \varepsilon)\right\|^{2} d x \leqslant M\|h\|^{2}
$$

для всех $h \in \mathfrak{H}$.

Шаг 2. Если ввести обозначение $\mathcal{F}(z, h)=\operatorname{row}\left\{\left(\left(I-z B^{*}\right)^{-1} g_{k}, h\right)\right\}_{k=1}^{n}$, то двойную оценку (3.29) можно переписать в виде

$$
m\|h\|^{2} \leqslant \int_{\mathbb{R}}\left\|\mathcal{F}(x+i \varepsilon, h) V^{-1}(-x+i \varepsilon)\right\|^{2} d x \leqslant M\|h\|^{2}
$$


для всех $h \in \mathfrak{H}$. Поскольку $V^{-1}(-x+i \varepsilon) \in \mathcal{M}_{n}^{2}$, в силу теоремы 1.3 существует оператор $S$, отображающий $\mathfrak{H}$ на некоторое подпространство пространства $\mathcal{K}_{a}$ и такой, что имеет место представление

$$
\mathcal{F}(x+i \varepsilon, h)=\mathcal{B}_{a}(x, S h), \quad h \in \mathfrak{H},
$$

отвечающее весу $w^{2}(x):=V^{2}(-x+i \varepsilon)$. Напомним, что через $а$ обозначается экспоненциальный тип резольвенты $\left(I-z B^{*}\right)^{-1}$. Из (3.30) и теоремы 1.2 вытекает, что оператор $S$ есть изоморфизм пространства $\mathfrak{H}$ на свой образ. Из неравенства (1.21) следует оценка

$$
\begin{aligned}
\int_{\mathbb{R}}\left\|\mathcal{F}(x, h) w_{-}^{-1}(x-i \varepsilon)\right\|^{2} d x & =\int_{\mathbb{R}}\left\|\mathcal{B}_{a}(x-i \varepsilon, S h) w_{-}^{-1}(x-i \varepsilon)\right\|^{2} d x \\
& \leqslant M\|S h\|_{\mathcal{K}_{a}}^{2} \leqslant M_{1}\|h\|^{2}, \quad h \in \mathfrak{H} .
\end{aligned}
$$

Напомним, что вес $w_{\varepsilon}^{2}(x):=w_{-}^{*}(x-i \varepsilon) w_{-}(x-i \varepsilon)$, где $w_{-}$участвует в факторизации (1.1), принадлежит классу $\mathcal{M}_{n}^{2}$. Оценку снизу мы выведем из представления (1.23) и ограниченности снизу оператора $T$, который определен равенством $\left(1.23^{\prime}\right)$. Итак, из (1.23) следует, что для любого $h \in \mathfrak{H}$

$$
\begin{aligned}
\int_{\mathbb{R}}\left\|\mathcal{F}(x, h) w_{-}^{-1}(x-i \varepsilon)\right\|^{2} d x=\int_{\mathbb{R}}\left\|\mathcal{B}_{a}(x-i \varepsilon, S h) w_{-}^{-1}(x-i \varepsilon)\right\|^{2} d x \\
\quad=\int_{0}^{\infty}\left\|e^{-\varepsilon t} G(S h, t)\right\|^{2} d t \geqslant e^{-2 \varepsilon a} \int_{0}^{a}\|(T S h)(t)\|^{2} d t \geqslant m_{1}\|S h\|_{\mathcal{K}_{a}}^{2} \geqslant m_{2}\|h\|^{2} .
\end{aligned}
$$

Последние два неравенства означают, что оператор $A$ есть $w$-возмущение оператора $A_{0}=\left(B^{*}\right)^{-1}$ с весом $w_{\varepsilon}^{2}$. Теорема доказана.

Отметим, что аналоги теоремы 3.4 имеют место для более широких классов операторов вида (3.26), однако это мы рассматривать не будем.

Выражения для операторов $U_{+}(t), U_{-}(t)$ вытекают из общих формул теории полугрупп. Например, пусть условия теоремы 2.3 выполняются при некотором $\varepsilon \geqslant 0$. Тогда справедлива следующая формула:

$$
U_{-}(t) h=-\frac{1}{2 \pi i} \int_{-\infty+i \varepsilon}^{+\infty+i \varepsilon} e^{-i z t} \sum_{k=1}^{n} f_{k}(h, z) A_{0}\left(A_{0}-z I\right)^{-1} g_{k} d z,
$$

где функционалы $f_{k}(h, z)$ определяются равенствами

$$
\begin{gathered}
f_{k}(h, z)=\sum_{j=1}^{n} \Psi_{k j}(z)\left(A_{0}\left(A_{0}-z I\right)^{-1} h, f_{j}\right), \\
\Psi(z):=\Phi^{-1}(z), \quad \Phi_{k j}(z)=\delta_{k j}-z\left(A_{0}\left(A_{0}-z I\right)^{-1} g_{j}, f_{k}\right), \quad 1 \leqslant k, j \leqslant n .
\end{gathered}
$$

Точные вычисления экспоненциальных типов полугрупп $U_{+}(t), U_{-}(t)$ мы приводить не будем. Укажем только, что такое вычисление, например для $U_{-}(t)$, основано на детальном изучении матрицы-функции $\Pi(z)$, которая фигурирует в формулировке следствия 2.1.

\section{§ 4. Примеры и некоторые применения доказанных теорем}

В этом параграфе мы изложим некоторые применения основных теорем о принадлежности полугрупп $U_{ \pm}(t)$ классу $C_{0}$. 
4.1. В гильбертовом пространстве $\mathfrak{H}$ рассмотрим оператор $A$, обратный к которому задается равенством

$$
A^{-1} h=B^{*} h+\sum_{k=1}^{n}\left(h, f_{k}\right) g_{k},
$$

где $B$ - вольтерров диссипативный оператор с $n$-мерной частью $\operatorname{Im} B$ и тривиальным ядром, системы векторов $\left\{f_{k}\right\}_{k=1}^{n},\left\{g_{k}\right\}_{k=1}^{n}$ образуют базисы пространства несамосопряженности $\mathcal{L}:=(\operatorname{Im} B) \mathfrak{H}$. Таким образом, существуют обратимые матрицы $C:=\left\|c_{k j}\right\|, D=\left\|d_{k j}\right\|$ такие, что

$$
f_{k}=\sum_{j=1}^{n} d_{k j} \varphi_{j}, \quad g_{k}=\sum_{j=1}^{n} c_{k j} \varphi_{j}, \quad 1 \leqslant k \leqslant n,
$$

где попарно ортогональные векторы $\varphi_{k}$ взяты из разложения

$$
\frac{1}{i}\left(B-B^{*}\right) h=\sum_{k=1}^{n}\left(h, \varphi_{k}\right) \varphi_{k}, \quad h \in \mathfrak{H} .
$$

Напомним, что элементы характеристической матрицы-функции $\Theta(z)$ оператора $B$ определяются равенствами

$$
\Theta_{j k}(z)=\delta_{k j}+i z\left((I-z B)^{-1} \varphi_{k}, \varphi_{j}\right), \quad 1 \leqslant k, j \leqslant n .
$$

Следующий результат доказывается путем применения теорем 2.3, 3.2 к операторам вида (4.1).

Теорема 4.1. Пусть $A$ - плотно заданный оператор в гилъбертовом пространстве $\mathfrak{H}$, обратнъй к которому задается формулой $(4.1), \Theta(z)$ - характеристическая матрица-функиия оператора В. Если при некотором $\delta>0$ элементы матрицы $e^{-i \delta z} \Theta(z)$ ограничены в $\mathbb{C}_{+}$, mо полугруппа $U_{-}(t)=\exp \{-i A t\}$ принадлежит классу $C_{0}$. Если, кроме того, матрица $E_{n}+i C D^{*}$ обратима, то полугруппа $U_{+}(t)=\exp \{i A t\}$ также принадлежит классу $C_{0}$.

ДокАЗАтЕльство. В силу теоремы 3.3 оператор $A$ является $w$-возмущением оператора $A_{0}=\left(B^{*}\right)^{-1}$ с весом $w^{2}(x) \equiv E_{n}, x \in \mathbb{R}$. Оператор $B^{*}$ унитарно эквивалентен своей функциональной модели (1.4) в пространстве $\mathcal{K}_{\Theta}$. Поэтому из теоремы 1.2 вытекает, что $w$-возмущению $A$ отвечают $E_{n} \in \mathcal{M}_{n}^{2}, \Theta(z) \in J_{n}$.

В результате простых выкладок с учетом формул (4.2) приходим к равенству

$$
{ }^{t} \Phi(z)=E_{n}+i C D^{*}-i C\left({ }^{t} \Theta(\bar{z})\right)^{*} D^{*},
$$

где $\Phi(z)$ - фигурирующая в формулировках теорем $2.3,3.2$ матрица-функция. Поскольку матрица $E_{n}+i C D^{*}$ обратима и $\lim _{y \rightarrow+\infty} \Theta^{*}(x+i y)=0$ равномерно по $x \in \mathbb{R}$, выполняются условия 1$)-3)$ теоремы 3.2 для некоторого $\varepsilon>0$, т. е. полугруппа $U_{+}(t)$ принадлежит классу $C_{0}$.

Поскольку $\Theta^{*}(\bar{z}) \Theta(z)=E_{n}$ [17], из (4.3) выводим равенство

$$
{ }^{t} \Phi(z)\left(D^{*}\right)^{-1}\left({ }^{t} \Theta(z)\right)=\Delta\left(D^{*}\right)^{-1}\left({ }^{t} \Theta(z)-i C\right), \quad \Delta:=E_{n}-i C D^{*},
$$

из которого следует, что $\sigma(A)$ лежит в некоторой области $\operatorname{Im} z<\varepsilon$. Кроме того, отсюда следует равенство

$$
\limsup _{y \rightarrow+\infty} y^{-1} \log (\Phi(i y) \Theta(i y))=0
$$


которое равносильно условию 2) теоремы 2.3 (этот факт установлен при доказательстве теоремы 2.3).

Далее, равенство (4.3) можно переписать в виде

$$
\Phi(z)=R+S \Theta^{*}(\bar{z}) T
$$

где $R, S, T$ - обратимые матрицы. Поскольку $\Theta^{*}(\bar{z})=\Theta^{-1}(z)$, в полуплоскости $\operatorname{Im} z>\varepsilon$, где $\Phi(z)$ обратима, получим

$$
\Phi^{-1}(z)=T^{-1} \Theta(z)\left(S^{-1} R T^{-1} \Theta(z)+E_{n}\right)^{-1} S^{-1} .
$$

Учитывая, что $e^{-i \delta z} \Theta(z)$ ограничена в $\mathbb{C}_{+}$и $\|\Theta(x+i b)\| \leqslant C(b)$ для всех $x \in \mathbb{R}[17]$, из равенств (4.4), (4.5) следует, что при достаточно больших $\varepsilon>0$ имеют место оценки

$$
\|\Phi(x+i \varepsilon)\| \leqslant M, \quad\left\|\Phi^{-1}(x+i \varepsilon)\right\| \leqslant M, \quad x \in \mathbb{R} .
$$

Они означают, что выполняется условие 3 ) теоремы 2.3. Теорема доказана.

Рассмотрим теперь один частный случай теоремы 4.1. Предположим, что $g_{k}=\sum_{p=1}^{n} Q_{k p} f_{p}, 1 \leqslant k \leqslant n$, где матрица $Q$ удовлетворяет условию $\operatorname{Im} Q \leqslant 0$. Тогда из (4.1) следует, что для любого $h \in \mathfrak{H}$

$$
\operatorname{Im}\left(A^{-1} h, h\right)=\operatorname{Im}\left(B^{*} h, h\right)+\operatorname{Im} \sum_{k=1}^{n} \sum_{p=1}^{n} Q_{k p}\left(f_{p}, h\right) \overline{\left(f_{k}, h\right)} \leqslant 0 .
$$

Таким образом, $\operatorname{Im}(A f, f) \geqslant 0$ для всех $f \in D_{A}$, и поэтому полугруппа $U_{+}(t)$ заведомо принадлежит классу $C_{0}$. С другой стороны, из (4.2) вытекает, что в этом случае $C=Q D$. Поскольку матрица $E_{n}+i C D^{*}=E_{n}+i Q D D^{*}$ всегда обратима, из теоремы 4.1 вытекает тот же результат, однако при дополнительном предположении ограниченности матрицы-функции $e^{-i \delta z} \Theta(z)$. Вместе с тем, доказательство теоремы 4.1 показывает, что это предположение является весьма грубым и, по-видимому, его можно заменить более слабым условием.

4.2. Напомним, что пространство Соболева $W_{2}^{1}\left([0, a], \mathbb{C}^{n}\right)$ состоит из вектор-функций $u(x)=\operatorname{col}\left(u_{k}(x)\right)_{k=1}^{n}$ с абсолютно непрерывными на отрезке $[0, a]$ координатами $u_{k}(t), 1 \leqslant k \leqslant n$. На этом пространстве задана норма

$$
\|u\|_{+}^{2}:=\sum_{k=1}^{n} \int_{0}^{a}\left|u_{k}(x)\right|^{2} d x+\sum_{k=1}^{n} \int_{0}^{a}\left|u_{k}^{\prime}(x)\right|^{2} d x<\infty .
$$

Рассмотрим систему $\varphi_{k}, 1 \leqslant k \leqslant n$, ограниченных в $W_{2}^{1}\left([0, a], \mathbb{C}^{n}\right)$ функционалов, удовлетворяющих условию нормировки:

$$
\varphi_{k}\left(e_{j}\right)=\delta_{k j}, \quad 1 \leqslant k, j \leqslant n,
$$

где $e_{j}=\operatorname{col}\left(\delta_{k j}\right)_{k=1}^{n}-$ стандартный орт, рассматриваемый как элемент пространства $W_{2}^{1}\left([0, a], \mathbb{C}^{n}\right)$. Предполагается, что система $\varphi_{k}$ линейно независима по $\bmod L_{2}^{n}(0, a)$, т. е. любая линейная комбинация $\sum_{k=1}^{n} c_{k} \varphi_{k}$ неограничена в $L_{2}^{n}(0, a)$. В силу последнего условия линеал

$$
\mathcal{L}:=\bigcap_{k=1}^{n} \operatorname{Ker} \varphi_{k}
$$

всюду плотен в $L_{2}^{n}(0, a)$. В пространстве $L_{2}^{n}(0, a)$ рассмотрим оператор

$$
A=i \frac{d}{d x}, \quad \mathcal{D}_{A}=\mathcal{L},
$$

а также оператор $A_{0}=i \frac{d}{d x}$, область определения которого состоит из всех 
функций $u \in W_{2}^{1}\left([0, a], \mathbb{C}^{n}\right)$ таких, что $u(0)=0$. Чтобы найти обратный оператор $A^{-1}$, решаем уравнение $i u^{\prime}(x)=h(x)$ с условием $u \in \mathcal{L}$. Поскольку

$$
u(x)=-i \int_{0}^{x} h(s) d s+\sum_{k=1}^{n} c_{k} e_{k},
$$

где $c_{k}$ - произвольные константы, из условий $\varphi_{k}(u)=0,1 \leqslant k \leqslant n$, с учетом (4.6) найдем

$$
c_{k}=\varphi_{k}\left(i \int_{0}^{x} h(s) d s\right), \quad 1 \leqslant k \leqslant n .
$$

Легко видеть, что функционалы $c_{k}$ ограничены в пространстве $L_{2}^{n}(0, a)$ и поэтому в силу теоремы Рисса допускают представления

$$
c_{k}(h)=\left(h, f_{k}\right), \quad f_{k} \in L_{2}^{n}(0, a), \quad 1 \leqslant k \leqslant n .
$$

Таким образом, из (4.8) следует равенство

$$
A^{-1} h=A_{0}^{-1} h+\sum_{k=1}^{n}\left(h, f_{k}\right) e_{k}, \quad\left(A_{0}^{-1} h\right)(x):=-i \int_{0}^{x} h(s) d s .
$$

В результате элементарных вычислений приходим к формуле

$$
\begin{gathered}
\mathcal{A}_{0}(z, h)=\operatorname{row}\left\{A_{0}\left(A_{0}-z I\right)^{-1} e_{k}, h\right\}_{k=1}^{n}=\operatorname{row}\left\{\int_{0}^{a} e^{-i z x} \overline{h_{k}}(x) d x\right\}_{k=1}^{n}, \\
h=\operatorname{col}\left(h_{k}\right)_{k=1}^{n},
\end{gathered}
$$

из которой следует, что $A$ есть $w$-возмущение оператора $A_{0} \in \Sigma^{(\exp )}$, которому отвечают постоянный $\left(A_{2}\right)$-вес $E_{n}$ и матрица-функция $\Theta(z)=e^{i a z} E_{n}$. Вычислим теперь соответствующую матрицу-функцию $\Phi(z)$. С учетом формулы (4.6), а также соотношений (4.9) и (4.10) найдем

$$
\begin{aligned}
\Phi_{k j}(z)=\delta_{k j}-z\left(A_{0}\left(A_{0}-z I\right)^{-1} e_{j}, f_{k}\right) & =\delta_{k j}-z \varphi_{k}\left(i \int_{0}^{a} e^{-i z s} e_{j} d s\right) \\
& =\varphi_{k}\left(e^{i z x} e_{j}\right), \quad 1 \leqslant k, j \leqslant n .
\end{aligned}
$$

Таким образом, применяя теоремы $2.3,3.2$, приходим к следующему результату.

Теорема 4.2. Пусть $A$ - произвольный оператор дифферениирования вида (4.7), $\Lambda$ - множество корней целой функиии $\Delta(z)=\operatorname{det} \Phi(z)$, где элементы матрици $\Phi(z)$ определяются по формулам $\Phi_{k j}(z)=\varphi_{k}\left(e^{-i z x} e_{j}\right), 1 \leqslant j, k \leqslant n$. Тогда:

1) полугруппа $U_{-}(t)=\exp \{-i A t\}$ принадлежит классу $C_{0}$ в том и только в том случае, когда при некотором $\varepsilon \geqslant 0$ выполняются условия:

(i) $\sup _{\lambda_{k} \in \Lambda} \operatorname{Im} \lambda_{k}<\varepsilon$;

(ii) $\lim \sup _{y \rightarrow+\infty} y^{-1} \log \mid \Delta($ iy) $\mid=n a$;

(iii) матричный вес $W_{\varepsilon}^{2}(x):=\Phi(x+i \varepsilon) \Phi^{*}(x+i \varepsilon)$ удовлетворяет $\left(A_{2}\right)$-условию на $\mathbb{R}$;

2) полугруппа $U_{+}(t)=\exp \{i A t\}$ принадлежит классу $C_{0}$ в том и только в том случае, когда при некотором $\varepsilon \geqslant 0$ выполняются условия:

(i) $\sup _{\lambda_{k} \in \Lambda} \operatorname{Im} \lambda_{k}>-\varepsilon$;

(ii) $\lim \sup _{y \rightarrow-\infty}|y|^{-1} \log |\Delta(i y)|=0$;

(iii) матричный вес $W_{\varepsilon}^{2}(x):=\Phi(x-i \varepsilon) \Phi^{*}(x-i \varepsilon)$ удовлетворяет $\left(A_{2}\right)$-условию на $\mathbb{R}$. 
Рассмотрим теперь в пространстве $L_{2}^{n}(0, a)$ задачу Коши

$$
\frac{d h(t)}{d t}=-i A h(t), \quad h(0)=h_{0} \in \mathcal{D}_{A},
$$

которой отвечает полугруппа $U_{-}(t)$. Предположим, что $U_{-}(t)$ принадлежит классу $C_{0}$. Поскольку $A$ порождается операцией $i \frac{d}{d x}$, каждая функция $h_{0} \in \mathcal{D}_{A}$ может быть единственным образом продолжена до функции $\tilde{h}(x), x \geqslant 0$, так, чтобы выполнялись условия:

a) $\tilde{h}(x)=h_{0}(x), 0 \leqslant x \leqslant a$;

b) $\tilde{h}(x+t) \in \bigcap_{k=1}^{n} \operatorname{Ker} \varphi_{k}$ для каждого $t>0$;

c) существует константа $M$ такая, что $\|\tilde{h}(x+a)\|_{L_{2}^{n}(0, a)} \leqslant M\|\tilde{h}(x)\|_{L_{2}^{n}(0, a)}$.

При этом решение задачи Коши (4.11) имеет вид

$$
h(t)=\tilde{h}(x+t), \quad 0 \leqslant x \leqslant a, \quad t>0 .
$$

Верно также обратное утверждение, т. е. если каждая функция $h_{0} \in \mathcal{D}_{A}$ допускает единственное продолжение $\tilde{h}$, удовлетворяющее условиям а)-c), то полугруппа $U_{-}(t)$ принадлежит классу $C_{0}$. Можно доказать, что условие с) является следствием условий a), b). Однако мы не будем здесь приводить это доказательство.

В случае $n=1$ аналогичные продолжения функций из пространства $C[0, a]$ за пределы интервала были введены Дж. Дельсартом и называются периодическими в среднем [27]. Поэтому продолжение функций вправо, удовлетворяющих условиям а)-c), естественно называть периодическим в среднем относительно системы функционалов $\varphi_{k}, 1 \leqslant k \leqslant n$. Рассматривая полугруппу $U_{+}(t)$, мы придем к понятию периодических в среднем продолжений вектор-функций влево.

Заметим, что каждый функционал $\varphi_{k}$ в силу теоремы Рисса представим в виде

$$
\varphi_{k}(u)=\sum_{j=1}^{n} \int_{0}^{a} u_{j}(x) g_{k j}(x) d x+\sum_{j=1}^{n} \int_{0}^{a} u_{j}^{\prime}(x) g_{k j}^{\prime}(x) d x
$$

где $u=\operatorname{col}\left(u_{k}\right)_{k=1}^{n}$, а элементы матрицы $G(x):=\left\|g_{k j}\right\|$ принадлежат пространству Соболева $W_{2}^{1}(0, a)$. Рассмотрим теперь систему интегро-дифференциальных уравнений со сдвигом аргумента:

$$
\int_{0}^{a} G(x) \tilde{h}(x+t) d x+\int_{0}^{a} G^{\prime}(x) \frac{d \tilde{h}(x+t)}{d x} d x=0, \quad \tilde{h}(x)=h_{0}(x), \quad 0 \leqslant x \leqslant a,
$$

где $h_{0} \in \mathcal{L}=\bigcap_{k=1}^{n} \operatorname{Ker} \varphi_{k}$.

Назовем задачу (4.12) корректной, если при каждом $h_{0} \in \mathcal{L}$ она имеет единственное решение $\tilde{h}$, которое удовлетворяет условиям а)-c). Ясно, что корректность задачи (4.12) равносильна принадлежности полугруппы $\exp \{-i A t\}$, где $A$ задается формулой $(4.7)$, классу $C_{0}$. Таким образом, первая часть теоремы 4.2 дает описание всех корректных задач (4.12). 
Из общей формулы (3.31) вытекает, что решение $\tilde{h}(x), x \geqslant 0$, корректной задачи (4.12) имеет вид

$$
\begin{aligned}
\tilde{h}(x)=\sum_{k=1}^{n}\left(-\frac{1}{2 \pi} \int_{-\infty+i \varepsilon_{1}}^{+\infty+i \varepsilon_{1}} e^{-i z x}\right. & \sum_{j=1}^{n} \Psi_{k j}(z) \varphi_{j} \\
& \left.\times\left(\int_{0}^{x} h_{0}(x-s) e^{-i z s} d s\right) d z\right) e_{k}, \quad x \geqslant 0 .
\end{aligned}
$$

Здесь предполагается, что условия первой части теоремы 4.2 выполняются при некотором значении $\varepsilon \geqslant 0$. Тогда в приведенной формуле $\varepsilon_{1} \geqslant \varepsilon$, при этом $\Phi^{-1}(z)=\left\|\Psi_{k j}(z)\right\|, \Phi(z)=\left\|\varphi_{k}\left(e^{-i z x} e_{j}\right)\right\|, e_{k}=\operatorname{col}\left(\delta_{k j}\right)_{j=1}^{k}$.

\section{Список литературы}

1. Т. Като, Теория возмущений линейных операторов, Мир, М., 1972; пер. с англ.: T. Kato, Perturbation theory for linear operators, Springer-Verlag, Berlin-HeidelbergNew York, 1966.

2. Э. Хилле, Р. Филлипс, Функиионалъный анализ и полугруппы, ИЛ, М., 1962; пер. с англ.: E. Hille, R. S. Phillips, Functional analysis and semigroups, Amer. Math. Soc. Colloq. Publ., 31, Providence, RI, Amer. Math. Soc., 1957.

3. С.Г. Крейн, Линейные дифференииальные уравнения в банаховом пространстве, Наука, M., 1967; англ. пер.: S. G. Krě̌n, Linear differential equations in Banach space, Transl. Math. Monogr., 29, Providence, RI, Amer. Math. Soc., 1971.

4. W. Arendt, Ch. J. K. Batty, M. Hieber, F. Neubrander, Vector-valued Laplace transforms and Cauchy problems, Monogr. Math., 96, Birkhäuser, Basel, 2001.

5. C. Chicone, Yu. Latushkin, Evolution semigroups in dynamical systems and differential equations, Math. Surveys Monogr., 70, Amer. Math. Soc., Providence, 1999.

6. K.-J. Engel, R. Nagel, One-parameter semigroups for linear evolution equations, Grad. Texts in Math., 194, Springer-Verlag, New York, 2000.

7. A. Pazy, Semigroups of linear operators and applications to partial differential equations, Appl. Math. Sci., 44, Springer-Verlag, New York, 1983.

8. Дж. Гарнетт, Ограниченные аналитические функиии, Мир, М., 1984; пер. с англ.: J. B. Garnett, Bounded analytic functions, Pure Appl. Math., 96, Academic Press, New York-London, 1981.

9. Г. М. Губреев, “Спектральная теория регулярных квазиэкспонент и регулярных $B$-представимых вектор-функций (метод проектирования: 20 лет спустя)", Алгебра и анализ, 12:6 (2000), 1-97; англ. пер.: G. M. Gubreev, "Spectral theory of regular quasiexponentials and regular $B$-representable vector-valued functions (the projection method: 20 years later)", St. Petersburg Math. J., 12:6 (2001), 875-947.

10. Б. С. Павлов, "Спектральный анализ диссипативного сингулярного оператора Шрёдингера в терминах функциональной модели", Дифференииалъные уравнения с частными производными - 8, Итоги науки и техн. Сер. Соврем. пробл. мат. Фундам. направления, 65, ВИНИТИ, М., 1991, 95-163; англ. пер.: В.S. Pavlov, "Spectral analysis of a dissipative singular Schrodinger operator in terms of a functional model", Partial differential equations VIII, Encyclopaedia Math. Sci., 65, Springer-Verlag, Berlin, 1996, 87-153.

11. Г. М. Губреев, Ю.Д. Латушкин, "Возмущения сильно непрерывных полугрупп операторов и матричные веса Макенхаупта", Функи. анализ и его прил., 42:3 (2008), 85-89; англ. пер.: G. M. Gubreev, Yu. D. Latushkin, "Perturbations of strongly continuous operator semigroups, and matrix Muckenhoupt weights", Funct. Anal. Appl., 42:3 (2008), 234-238. 
12. S. Treil, A. Volberg, "Wavelets and the angle between past and future", J. Funct. Anal., 143:2 (1997), 269-308.

13. D. Z. Arov, H. Dym, J-contractive matrix valued functions and related topics, Encyclopedia Math. Appl., 116, Cambridge Univ. Press, Cambridge, 2008.

14. Б. Секефальви-Надь, Ч. Фояш, Гармонический анализ операторов в гильбертовом пространстве, Мир, М., 1970; пер. с фр.: B. Szőkefalvi-Nagy, C. Foiaş, Analyse harmonique des operateurs de l'espace de Hilbert, Masson et Cie, Paris, 1967.

15. В. А. Золотарев, Аналитические методъ спектралъных представлений несамосопряженных и неунитарных операторов, Из-во Харьковского национальн. ун-та, Харьков, 2003.

16. В.П. Потапов, "Мультипликативная структура $J$-нерастягивающих матрицфункций”, Тр. МMO, 4, Физматлит, М., 1955, 125-236; англ. пер.: V.P. Potapov, "The multiplicative structure of J-contractive matrix functions", Amer. Math. Soc. Transl. (2), 15 (1960), 131-243.

17. М. С. Бродский, Треугольные и жордановы представления линейных операторов, Наука, M., 1969; англ. пер.: M. S. Brodskii, Triangular and Jordan representations of linear operators, Amer. Math. Soc., Providence, RI, 1971.

18. S. A. Avdonin, S. A. Ivanov, Families of exponentials. The method of moments in controllability problems for distributed parameter systems, Cambridge Univ. Press, Cambridge, 1995 .

19. Б. Я. Левин, Распределение корней целых функиий, Гостехиздат, М., 1956; англ. пер.: В. Ja. Levin, Distribution of zeros of entire functions, Amer. Math. Soc., Providence, RI, 1964.

20. Б. Я. Левин, Ю. И. Любарский, "Интерполяция целыми функциями специальных классов и связанные с нею разложения в ряды экспонент", Изв. АН СССР. Сер. матем., 39:3 (1975), 657-702; англ. пер.: В. Ya. Levin, J. I. Ljubarskiǐ, "Interpolation by means of special classes of entire functions and related expansions in series of exponentials", Math. USSR-Izv., 9:3 (1975), 621-662.

21. А. М. Седлецкий, “Эквивалентное определение пространств $H_{p}$ в полуплоскости и некоторые приложения", Матем. сб., 96(138):1 (1975), 75-82; англ. пер.: A. M. Sedleckiǐ, "An equivalent definition of $H_{p}$ spaces in the half-plane and some applications", Math. USSR-Sb., 25:1 (1975), 69-76.

22. И. М. Гельфанд, "Об одной лемме теории линейных пространств", Сообщ. Харъковского мат. общества, 13:4 (1936), 35-40.

23. Н. И. Ахиезер, И. М. Глазман, Теория линейных операторов в гилъбертовом пространстве, Вища школа, Киев, 1977; англ. пер.: N. I. Akhiezer, I. M. Glazman, Theory of linear operators in Hilbert space, Boston-London, Pitman, 1981.

24. Z.D. Arova, Operator nodes with strongly regular characteristic functions, Huisdrukeruj Vrije Universitet, Amsterdam, 2003.

25. Н.К. Никольский, Лекиии об операторе сдвига, Наука, М., 1980; англ. пер.: N.K. Nikol'skiı̌, Treatise on the shift operator, Grundlehren Math. Wiss., 273, Springer-Verlag, Berlin-Heidelberg, 1986. 
26. И.Ц. Гохберг, М.Г. Крейн, Введение в теорию линейных несамосопряженных операторов в гилъбертовом пространстве, Наука, М., 1965; англ. пер.: I. C. Gohberg, M. G. Kreln, Introduction to the theory of linear nonselfadjoint operators, Transl. Math. Monogr., 18, Amer. Math. Soc., Providence, RI, 1969.

27. J. Delsarte, "Les fonctions "moyenne-périodiques", J. Math. Pures Appl., 14 (1935), 403-453.

Г. М. Гувреев (G. M. Gubreev)

Полтавский национальный технический университет им. Ю. Кондратюка, Украина

E-mail: gubreev@yandex.ru

Ю. Д. ЛАтУШКИн (Yu. D. LAtushkin)

University of Missouri-Columbia, USA

E-mail: yuri@math.missouri.edu
Поступило в редакцию 16.03.2009 